\title{
Dedicated Poster Abstracts
}

Published: April 18, 2020

CMEJ 2020, e155-e274 Available at http://www.cmej.ca

(C) 2020; 11(2) licensee Synergies Partners

https://doi.org/10.36834/cmej.70106

This is an Open Journal Systems article distributed under the terms of the Creative Commons Attribution License

(http://creativecommons.org/licenses/by/2.0) which permits unrestricted use, distribution, and reproduction in any medium, provided the original work is properly cited.

\section{Sunday, April 19th - 10:00-11:30}

\section{Dedicated Poster Session}

\section{DP 1-1 Medical Student Perceptions of a} \section{Case-Based Virtual Dissection Anatomy}

\section{Curriculum}

Bruce B. Forster University of British Columbia, Anique de Bruin Maastricht University, Abigail Arnold University of British Columbia, Rose Hatala University of British Columbia, Jeroen van Merrienboer Maastricht University, Kathryn Darras University of British Columbia, Rebecca Spouge University of British Columbia, Savvas Nicolaou University of British Columbia

Background/Purpose: Virtual dissection is performed with CT scans on near life-size touchscreens, which function like hospital radiology workstations. Students work together to manipulate the data and perform their dissection. The purpose of this study was to develop and qualitatively assess the educational value of virtual dissection laboratories for first year medical students as well as to understand students' preferred pedagogical approaches for learning from this new technology.

Methods: 105 first-year medical students participated in a case-based virtual dissection curriculum and were invited to complete a theorybased post-experience survey. Eight unique cases were selected based on the first-year curricular objectives and in groups of 6-8, students reviewed the cases with a radiologist. First, students' reactions to virtual dissection were measured by three constructs using a 5-point Likert scale: quality of curriculum design (11 questions), impact on learning (7 questions), and comfort with technology (3 questions). Second, students ranked the usefulness of six pedagogical approaches for this technology. Responses were tabulated and rank order item lists were generated statistically using the Schulze method where appropriate.

Results: The survey response rate was $83 \%$ (87/105). Overall, students' reactions to virtual dissection were positive across all three measured constructs. Most students indicated that the cases were of an appropriate level of difficulty (90\%) and that virtual dissection improved their understanding of disease and pathology (89\%), the clinical relevance of anatomy (77\%), and visuospatial relationships (64\%). Almost all students (94\%) reported that the curriculum improved understanding of the role of the radiologist in patient care. Students felt that the "very useful" pedagogical approaches were small group demonstration (68\%) and problem-based learning (51\%).

Conclusion: First-year medical students perceive virtual dissection as a valuable tool for learning anatomy and radiology. This technology allows reallife clinical cases to be introduced into pre-clinical learning, thereby providing opportunities to strengthen the vertical integration in medical curricula. 


\section{DP 1-2 Using a Digital Multimedia Platform to Teach Anatomical Sciences}

Kamal Moghrabi University of British Columbia, Majid Doroudi University of British Columbia

Background/Purpose: The Doctor of Medicine Undergraduate Program (MDUP) at the University of British Columbia has employed anatomy procedure videos to prepare students ahead of attending gross anatomy laboratory sessions. The theory is that students who watch these video tutorials will be better prepared to maximize learning from the laboratory sessions, thus using this time more effectively to achieve learning outcomes. This project produced a video to support the MDUP video series, offering a gross anatomy laboratory dissection guide to outline and present significant anatomical and physiological components of the chest and lungs. Through student survey feedback, we determined the usefulness of videos in helping students achieve anatomy learning objectives and which areas of the videos were most helpful in doing so. If successful, the content of this video production can also be incorporated into the curriculum of other disciplines (healthcare related or otherwise) including, but not limited to, the physical therapy programs, doctor of dental medicine (DMD) programs, bachelor of nursing programs (BSN), and undergraduate physiology and kinesiology degree programs.

Summary of the Innovation: A series of anatomy video tutorials were uploaded to an official UBC FLEX YouTube account between 2017 and 2019. Survey results were collected and collated. Medical student feedback was overwhelmingly positive as most participants found the videos to be effective in achieving learning outcomes.

Conclusion: This study serves as a foundation for future work in the development of multimedia to teach anatomical sciences. The content of these video productions can be incorporated into the curriculum of other disciplines (healthcare related or otherwise).
DP 1-3 Medical students' perceptions of anatomy teaching in the medical undergraduate curriculum: Cadaveric vs radiologic learning

Kathryn Darras University of British Columbia, Juvel Lee University of British Columbia, Anique de Bruin Maastricht University, Savvas Nicolaou University of British Columbia, Bruce Forster University of British Columbia

Background/Purpose: Radiology aids patient diagnosis and management. All physicians should be able to provide preliminary interpretations of imaging studies. Thus many medical undergraduate programs are integrating radiology into anatomy curricula to better prepare students for clinical practice. To date, no research has assessed the ideal balance between cadaveric and radiologic content in anatomy teaching. This study aims to assess undergraduate medical students' ("UMS") comfort level in identifying cadaveric and radiologic anatomy, and to identify areas where curricula can be renewed.

Methods: An anonymous online survey was sent to years 2-4 UMS at a large distributed medical school teaching cadaveric and radiologic anatomy. Respondents' demographic information and prior radiology exposure was collected. UMS ranked their comfort level in identifying cadaveric and radiologic anatomy on a 4-point Likert scale. Statistical analysis was done to determine any difference in responses by stage of training. Copeland score method generated a rank-order list of UMS comfort level across all variables.

Results: 153 UMS did the survey (response rate 18\%). Respondents: $50.3 \%$ female; $51.6 \%$ in clinical years; $90.8 \%$ reported no radiology teaching before medical school; $65.4 \%$ rated their overall anatomy knowledge as "average." Likert data showed UMS felt most comfortable with identifying cadaveric anatomy (organs, bones, spatial relationships) and least comfortable with radiologic anatomy (pathology, neuroanatomy, spatial relationships). No statistical difference with training level.

Conclusion: UMS reported feeling less comfortable with identifying anatomy on radiologic studies compared to the same structures in cadavers. This 
study suggests that UMS would benefit from more exposure to radiology anatomy during medical school.

\section{DP 1-4 Reimagining dissection lab preparation - the role of digital media in anatomy education}

Farnaz Javadian University of British Columbia, Andy Jiang University of British Columbia, Nicole Ng University of British Columbia, Shirley Tse University of British Columbia, Majid Doroudi University of British Columbia

Background/Purpose: Learning materials provided to students prior to anatomy dissection labs have classically been in the form of written instructions and images from prosections. However, these materials can be difficult to interpret, especially for beginner students. With the advancement of technology, educational curricula are exploring the integration of digital platforms to supplement more traditional teaching methods. The aim of this project was to investigate the utility of a video-based guide for approaching dissection.

Summary of the Innovation: A video dissection guide was created demonstrating the dissection of the superficial back. The video outlines anatomy, technique, procedure, and includes review questions. The video was made available online through YouTube, along with a feedback survey.

Conclusion: After one month, the video has received more than 1,000 views. Feedback was received from 65 respondents; a majority of whom were female (63.1\%), aged 20-24 (58.5\%), and had a current education level of a postgraduate degree or professional degree $(53.8 \%)$. The majority of respondents agree or strongly agree that the video presented the anatomy in a clear and organized fashion (95.4\%), enhanced their learning of the anatomy $(100 \%)$, familiarized them with dissection tools and how to use them (89.2\%), familiarized them with methods and techniques for dissection (92.3\%), was more effective than a dissection guide (96.9\%), and was more effective for learning anatomy than a textbook (87.7\%). A poll of fifty-one respondents found that most agree or strongly agree that the video is a valuable resource for review/test preparation (88.2\%). In summary, digital media in the form of video-based dissection guides may be a useful tool to incorporate into educational curricula for teaching gross anatomy.

\section{DP 1-5 Assessing the feasibility of peer-} teaching in a first-year medical student virtual dissection curriculum

Nicole Ng University of British Columbia, Andy Jiang University of British Columbia, Bruce Forster University of British Columbia, Savvas Nicolaou University of British Columbia, Jeffrey Hu University of British Columbia, Rebecca Spouge University of British Columbia, James Nugent University of British Columbia, Kathryn Darras University of British Columbia.

Background/Purpose: Virtual dissection is a novel teaching method using radiological images to study human anatomy. We aimed to determine the educational value and student satisfaction with a peer-to-peer teaching model using this emerging technology

Summary of the Innovation: Second year medical students prepared and taught virtual dissection laboratories to first-year students, which were voluntary and extra-curricular. The case-based laboratories were designed to expose participants to clinical radiology. Participants completed a postlaboratory survey based on the Kirkpatrick Hierarchy for curriculum evaluation.

Conclusion: Thirty-four first year students (24 females, 10 males) participated in this laboratory. All respondents found the cases were presented an appropriate level of difficulty, with $94 \%$ of participants believing the peer tutor facilitated the session effectively. Upon completing the session, the majority of participants felt that they understood the imaging findings (88\%), clinical cases (91\%), and perceived the session as a valuable learning experience (97\%). Participants agreed or strongly agreed that the sessions improved their understanding of anatomy (97\%) and disease (85\%), the appearance of normal (94\%) and pathological (97\%) structures, and visuospatial relationships (91\%). Almost all participants (97\%) agreed or strongly agreed that the session was a valuable 
learning experience with a majority preferring the small group format versus large group teaching. In summary, students reported that peer-to-peer teaching was a valuable learning experience in virtual dissection laboratories. Peer-to-peer based virtual dissection laboratories provide an innovative and collaborative mode of learning that can complement more traditional anatomy education methods.

\section{DP 1-6 Integrated virtual and cadaveric dissection laboratories enhance first year medical students' anatomy experience: A pilot study}

Kathryn Darras University of British Columbia, Rebecca Spouge University of British Columbia, Rose Hatala University of British Columbia, Savvas Nicolaou University of British Columbia, Jeff $\mathrm{Hu}$ University of British Columbia, Anne Worthington University of British Columbia, Claudia Krebs University of British Columbia, Bruce B. Forster University of British Columbia

Background/Purpose: Radiology integration into medical anatomy programs is well established, but there is a paucity of literature on integrating virtual dissection into cadaveric dissection laboratories. Virtual dissection is the digital dissection of medical images on touchscreen anatomy visualization tables. The purpose of this pilot study was to investigate the feasibility of integrating virtual dissection into a firstyear medical cadaver-based anatomy course and to assess students' overall attitude towards this new technology.

Methods: All students in first-year medicine at a single medical school participated in this study ( $\mathrm{n}=$ 292). Six virtual dissection laboratories, which focused on normal anatomy, were developed and integrated into a cadaver-based anatomy course. The virtual dissection table (VDT) was also integrated into the final anatomy spot exam. Following the course, students completed a short evidence-informed survey which was developed using a theoretical framework for curriculum evaluation. Numerical data were tabulated, and qualitative content analysis was performed on students' unstructured comments.

Results: The survey response rate was 69.2\% ( $\mathrm{n}=$ 202/292). Most (78.7\%) students reported that virtual dissection enhanced their understanding of the cadaveric anatomy and the clinical applications of anatomy. Most (73.8\%) students also felt that the VDT was an effective use of the laboratory time. Thirteen narrative comments were collected, most of which (61.5\%) identified strengths of the curriculum.

Conclusion: In this pilot study, students perceived that their learning was enhanced when virtual dissection was combined with a traditional cadaverbased anatomy laboratory. This study demonstrates that there is potential for virtual dissection to augment cadaveric dissection in medical education.

DP 1-7 Anatomy in a New Curriculum: Using Digital Media to Facilitate the Learning of Anatomy in the Medical Curriculum

Vishesh Oberoi University of British Columbia, Farshad Hosseini University of British Columbia, Majid Doroudi University of British Columbia

Background/Purpose: The advances in technology have allowed teachers to develop new ways to teach their course materials to their students. This has ranged from using educational videos to developing online learning modules that students may complete at their pace and time. Although these advances in enhancing student knowledge have been made in many subjects, there are limited resources available to students for the purposes of learning anatomy. One area that needs to be expanded is one of educational anatomy video tutorials/lessons. This project was designed to create an educational video tutorial/lesson that provides a step by step tutorial that the students can follow to complete their anatomy lab. The video is designed to complement the lab component rather than replace it as we have realized that there is no substitute for viewing the material first hand. The video would allow students to prepare for the lab and upon its completion, they may refer back to the video and use it as a study tool.

Methods: To begin the cadaver was dissected and prepared so that the anatomical structures that needed to be identified were properly exposed for the filming. The filming was done through a stepwise manner, where the anatomical structures were identified and commented on throughout the video. During the editing, process animations were added to 
tag key anatomical structures and a quiz was added towards the end to allow students to test their knowledge on the materials in the video.

Results: To gather the efficacy of the videos and receive feedback we gave the students a survey to conduct prior to and after their lab. The results showed that more than $95 \%$ of students felt that the video helped them prepare for the upcoming lab dissection. While $98.9 \%$ of students felt that they would like to have a similar lab video for labs in the future. Also, 93\% of the students stated that they would like to use the videos to study for their exams.

Conclusion: Due to the positive responses received it can be concluded that the use of video tutorials in teaching anatomy is an effective tool that can be implemented alongside the lecture material and cadaver dissections to enhance the learning of students.

\section{DP 2-1 OAST at MUN Medicine: An} innovative case of service learning and community partnership

Richard Berish Memorial University of Newfoundland, Josh Lehr Memorial University of Newfoundland, Matthew Downer Memorial University of Newfoundland, Luke Duffley Memorial University of Newfoundland, Phil Hillier Memorial University of Newfoundland, Kieran LaceyMemorial University of Newfoundland, Madison LewisMemorial University of Newfoundland, Brooke TurnerMemorial University of Newfoundland, Jill AllisonMemorial University of Newfoundland

Background/Purpose: MUN Medicine's Opioid Awareness and Support Team (OAST) is a student run organization created in 2017. OAST's objectives are to address knowledge gaps among students and the community surrounding opioid use and treatment. One central tenant is to focus on service learning, where students engage with community partners for bilateral learning and mutual benefit.

Summary of the Innovation: OAST has engaged three different community organizations to both provide students with increased knowledge of opioid use/harm reduction as well as disseminate important information to the community. OAST's partnership with the local Safe Works Access Program (SWAP) provided students information about harm reduction to students and Naloxone training sessions, while students worked with SWAP to pack harm reduction kits. Student feedback and reflection indicates increased awareness and understanding of harm reduction. Second, in partnership with a local organization for marginalized youth, we developed and delivered Opioid Awareness Workshops. Surveys following the workshops indicates that the need for information was met. Lastly, OAST partnered with the local penitentiary to discuss opioid related health issues with incarcerated individuals. We will deliver our workshop to staff at the penitentiary in the future and work with other community organizations who identify a similar need for information.

Conclusion: OAST has provided students will novel service learning opportunities as well as connected community organizations with informed students to teach on a critical public health topic. Follow up with community partners indicates positive responses regarding impact and mutual benefit. OAST is an example of a student led initiative in community partnership that is knowledge driven and strengthens capacity at the local level.

DP 2-2 A policy review of social accountability: Implications for evaluation in medical training

Cees van der Vleuten Maastricht University, Jimmie Leppink University of York, Cassandra Barber Maastricht University, Saad Chahine Queen's University

Background/Purpose: Social accountability (SA) is central to medical education. While many institutions strive to be accountable to the communities they serve, the extent to which programs meet societal needs remains largely unknown. Part of this problem may be a result of the operational complexities surrounding SA. This paper provides the initial operational concepts needed to evaluate SA in medical training through reviewing and synthesizing SA policies.

Methods: Thematic synthesis was used to identify operational concepts from large-scale, public SA policies. Common and unique elements across policies were thematically coded using context-input- 
process-products (CIPP) evaluation model as an organizational framework.

Results: Out of an initial sample of 32 documents, four large-scale SA policy frameworks were selected and analyzed. Sub-policies and/or program/institution specific documents were excluded from the analysis as they build upon previous frameworks and may lack generalizability. Emerging themes highlighted across policies included core social values. These values were expected to be displayed across the training continuum. Sub-themes under each CIPP domain included: context (public display of institutional goals/mandates, collaborative partnerships with health systems, contribution to health policy); inputs (diversity/equity in recruitment/selection, community health profiles); processes (curricular activities, learning opportunities/exposure); products (physician resource planning, quality assurance, evaluation/accreditation) and impacts (improve community health, reduce/prevent health risks, morbidity/mortality).

Conclusion: Using an evaluation framework, this paper provides a thematic synthesis of key SA frameworks. Findings from our analysis revealed major themes consistent to the broader literature. However, this paper operationalized a set of performance indicators that can be used to guide programs evaluate SA.

\section{DP 2-3 Teaching Medical Learners about the}

Social Determinants of Health for Improved

\section{Patient Treatment at the Bedside}

Meghan Gipson University of Toronto, Lisa Richardson MD PhD University of Toronto

Background/Purpose: The "Social Determinants of Health" (SDoH) is the term for the factors that impact the emotional and physical wellbeing of patients. These factors contribute greatly to inequity of patient healthcare experiences. Yet, many healthcare professionals do not recognize the unique life circumstances of their patients, or how such circumstances warrant different treatment approaches. The World Health Organization, the Association of American Medical Colleges, and the Royal College of Physicians and Surgeons of Canada, among others, have identified the importance of teaching medical trainees how to act on the SDoH to effectively care for patients.

Methods: This poster reviews literature on programs for teaching social medicine. It then outlines the best practices for teaching medical learners how to account for the SDoH when delivering patient care. MEDLINE at OVID, Embase, and Cinahl Plus were searched for the terms: "Social Determinants of Health," and "teaching," or "students, medical/ students, health occupations" with no age, publication date, or language restrictions. Teaching strategies from relevant articles were discussed with Toronto-based physicians for their input.

Results: A curriculum for SDoH-based teaching techniques to enact meaningful change in the provision of care was developed and is discussed in this poster. The literature identifies that, despite the growing number of programs addressing $\mathrm{SDoH}$ in their medical curricula, the programs do not adequately teach medical learners how to address the influence of the $\mathrm{SDoH}$ on the patient when delivering care at the bedside.

Conclusion: Accordingly, the curriculum guidelines in this poster have been developed to address this gap in the education of medical students - to ensure that a patient's social circumstances inform their treatment.

\section{DP 2-4 Voisins de la Rue}

Claudianne Laurin Université de Montréal, Marie-Ève Villeneuve Université de Montréal, Camille Dulude Université de Montréal

Background/Purpose: L'incohérence entre la demande de soins de santé de la population vulnérable et l'accès offert par la communauté médicale est inconcevable. Pour les étudiants, la création de liens avec cette clientèle est confrontante. Les préjugés peuvent nuire à la qualité de la relation, alors que cette dernière est essentielle à une démarche de soins efficace et inclusive. L'initiative étudiante "Voisins de la rue " offre l'opportunité d'aborder la réalité complexe des populations vulnérables dans un contexte sécurisant et encadré. Non seulement ce projet permet de valoriser la responsabilité sociale du futur médecin; 
mais il contribue à développer l'autonomie et l'estime personnelle chez les personnes marginalisées de Trois-Rivières, étant l'une des dix villes les plus pauvres au Québec.

Summary of the Innovation: En septembre 2017, trois étudiantes de l'UdeM au campus décentralisé de la Mauricie ont d'abord collaboré avec les intervenants d'organismes communautaires locaux. Ensemble, ils ont identifié les besoins des usagers et créé des activités rejoignant des objectifs variés : art, cuisine, musique, relaxation et jeux. Annuellement, chacune des 7 activités, où la communication est priorisée, permet d'établir des liens significatifs entre 5 à 8 étudiants et un même nombre de participants marginalisés. De plus, une collecte de dons est effectuée à chaque Noël pour les gens en situation d'urgence. À ce jour, 17 \% des étudiants entre l'année préparatoire et la fin du préclinique ont participés.

Conclusion: Au quotidien, les étudiants sont sensibilisés à la réalité des personnes vulnérables et peuvent adopter une approche médicale personnalisée et collaborative. En diffusant cette initiative au Canada, l'importance d'un accès aux soins égal pour tous sera mis de l'avant.

\section{DP 2-5 Fostering Advocacy Among Medical}

Learners Through Community Based
Service Learning: A Scoping Study

Jasmine Chahal University of Toronto, Mitesh Patel University of Toronto

Background/Purpose: Despite widespread acceptance of advocacy as a professional obligation over recent years (Frank, 2005), teaching and evaluating health advocacy has been challenging (Poulton \& Rose, 2015). Service learning has emerged as a valuable educational tool for fostering knowledge and skills in community health and raising awareness of social determinants of health amongst medical trainees (Buckner, 2010). Despite this, medical schools continue to be challenged in finding ways to effectively incorporate service learning and advocacy into their curriculum and measure the outcomes of those initiatives on training physicians as health advocates. Through our study, we aimed to identify current educational strategies and research gaps in fostering advocacy amongst medical students and residents in the context of community-based service learning (CBSL).

Methods: Scoping review procedures were applied to systematically search, screen, extract and synthesize this broad body of literature following the Preferred Reporting Items for Systematic Reviews and MetaAnalyses guidelines.

Results: Ten key themes related to strategies and approaches to advocacy education were identified within 68 selected publications: (a) geographic location b) community partners (c) community populations served (d) program participants (e) program format (f) evaluation (f) data analysis (g) results and student outcomes (h) sustainability (i) challenges and limitations.

Conclusion: This scoping review provided key insights into a variety of CBSL advocacy program formats, evaluation methods, and outcome measures which can be used as a framework for establishing sustainable programs aimed at fostering advocacy amongst medical trainees. A list of recommendations for future areas of inquiry is also provided to guide future research.

\section{DP 2-6 Evaluating the effectiveness of a} 'Muslims in Healthcare' symposium on improving the cultural competence of healthcare trainees

Shaimaa Helal Queen's University, Emaad Mohammad Queen's University, Omar Islam Queen's University

Background/Purpose: Muslims represent the second largest religious group in Canada. Western Muslim patients share religiously informed healthcare values. The inadequate assessment of cultural needs has been shown to contribute to a lower quality of care for Muslim patients in Western countries. Studies have shown that cultural competence training is associated with improved cultural competence of healthcare providers, increased patient satisfaction and reduction in health disparities for priority populations. This study aimed to assess the effectiveness of a didactic-style symposium at improving the cultural competence of healthcare trainees in the care of Muslim patients. 
Methods: 44 healthcare trainees from Queen's University attended a three-hour symposium on topics related to caring for Muslim patients. Students completed a modified version of the cultural competence self evaluation (CCSE) form pre- and post-symposium. Descriptive statistics were calculated using means (SD) for continuous variables and frequencies (\%) for categorical variables. Students' pre and post-CCSE scores, and the score's three sub-components were compared with a paired t-test.

Results: The average CCSE (out of 80 ) was $36 \pm 9.03$ pre-, and $49.71 \pm 10.84$ post-symposium. Participants improved a mean of $13.71 \pm 8.34$ points on the CCSE $(p<0.001)$. This represents an improvement from "incompetent" to "near competent". Participants showed an improvement of $9.30 \pm 6.31$ points on the knowledge component ( $p<0.001), 4.30 \pm 3.55$ points on the ability component $(p<0.001)$ and $0.11 \pm 1.02$ $(p=0.462)$ on the desire component.

Conclusion: Healthcare trainees show an interest and need to improve their cultural competence skills. This symposium was effective at improving self-perceived knowledge of and ability to interact with Muslim patients.

\section{DP 2-7 A Truth and Reconciliation} Commission (TRC) Report Reading Group Applying TRC's Calls to Action to Medical Students

Aarti Sayal University of Toronto

Background/Purpose: In 2015, The Truth and Reconciliation Commission (TRC) of Canada released a report to inform all Canadians about the truth of Indian Residential Schools. Along with this report, "Calls to Action" were created to advance the process of Canadian reconciliation, including actions related specifically to health. One of the "Calls to Action" asks for medical students to take a course dealing with Indigenous health, including the history of residential schools. Hence, it is clear that the need for medical students to understand the history of residential schools is vital to addressing health disparities seen among Indigenous peoples today. Unfortunately, many medical students in Canada are not aware of this full history, and have not read the report put forward by TRC. Therefore, a TRC Report Reading Group for medical students has been created at the University of Toronto, as a student-led initiative, which is the first of its kind to be introduced in Canadian medical schools.

Summary of the Innovation: The goal is to create, implement, and evaluate a TRC Reading Group to 1) inform medical students of the truth behind Indian Residential Schools to help reduce health inequities seen among Indigenous peoples and 2) address the "Calls to Action" released by the TRC. In collaboration with First Nations' House, the Medical Society's Global Health Portfolio, and the Office of Indigenous Medical Education at the University of Toronto, a TRC Report Reading Group was created. The TRC Reading Group was conducted over three sessions each lasting an hour, at which an Elder was present to facilitate. Medical students were asked to read certain sections of the 500-page Executive TRC Summary report prior to each session. During each session, reflective discussions on the contents of the report occurred, as well as a discussion on future steps medical students and physicians can take to provide the best care to Indigenous patients. Furthermore, an iterative process was used to develop these sessions, meaning that the content and discussion of the group was adapted over the course of the three sessions. This was done by ensuring there was feedback from both the Elder and students to help guide how each session would run.

Conclusion: There is a critical need for learning about Indigenous health through experiential and reflective pedagogical techniques. Examples include a blanket exercise and learning from Elders. Ultimately, the TRC Reading Group is another approach where medical students can safely and deeply consider the experiences of Indigenous Peoples of Canada and their future roles of addressing Indigenous Peoples' ongoing inequities in healthcare. 
DP 2-8 We have work to do! Medical learners and speaking up to positions of power

Jackie Gruber University of Manitoba, Lukas Neville University of Manitoba, Cathy Rocke University of Manitoba, Brenda Elias University of Manitoba

Background/Purpose: In medical education the learner environment has been identified as an area for improvement. Environments where mistreatment exists can have negative consequences for learning. For instance, if learners do not feel safe to speak up, if they are fearful of being humiliated by someone in a position of power, or if they have concerns about negative career consequences. Mistreatment can also limit learners in reporting patient safety issues therefore, silence and self-censorship may have serious consequences. This study sought to identify medical learners' comfort level in speaking up to faculty members, nurses, residents, administrators and patients.

Methods: A cross-sectional online survey targeted 440 UGME learners at the University of Manitoba, (32\% participation rate). After data editing, a logistic regression analysis was conducted on a sample of 123 learners.

Results: Male learners were more comfortable then female learners in speaking up to faculty members $(O R=4.5)$, nurses $(O R=2.6)$, residents $(O R=2.4)$ and administrators $(\mathrm{OR}=4.8)$. Caucasian learners were more comfortable speaking up to administrators $(O R=2.8)$ than racialized learners. First generation learners were less comfortable speaking up to both patients $(O R=-2.9)$ and residents $(O R=-2.9)$.

Conclusion: This study showed that women learners, racialized learners, and first-generation learners were less likely to be comfortable speaking up to positions of power. Given the increasing diversity of medical learners and the importance of speaking up regarding mistreatment, this is an important finding. It suggests a need for interventions to increase learner comfort with speaking up, and to address the conditions leading to the observed gender disparity.

\section{DP 3-1 Development of the Edmonton Frail Scale Training Course}

Hartley Perlmutter University of Alberta, Darryl Rolfson University of Alberta, Thomas Jeffery University of Alberta, Vijay Daniels University of Alberta, Patrick von Hauff University of Alberta

Background/Purpose: The Edmonton Frail Scale (EFS) is a multidimensional tool for assessing frailty, that both produces a single score reflective of a patient's overall frailty status and identifies specific aspects of frailty that warrant follow up. Over the past two years, steps have been taken to prepare the EFS for implementation in clinical settings, including the creation of a training video and an "EFS Toolkit" to train health care professionals to administer the EFS. The next step in implementation was to create a training course.

Summary of the Innovation: Our final product is a 12module training course, with accompanying practice activities and a simulation-based final assessment to assess the trainee's ability to administer the EFS in a home care setting. Using the educational framework of Backward Design, we mapped out course content and assessment. We then utilized Kane's framework to validate the simulation-based assessment. We conducted six feedback sessions with care providers to ensure that course instruction is understandable, and that the simulation reflects an authentic patient encounter.

Conclusion: The success of this innovation was the result of strong grounding in conceptual frameworks in a collaborative environment. The Backward Design framework helped us align the course content and assessment with our course objectives, and Kane's framework helped us provide validity evidence for the assessments. Our project was also enhanced by the collaborative work environment in the University of Alberta's Faculty of Medicine and Dentistry Academic Technologies studio, where educators, medical students and designers take advantage of complimentary but very different expertise. 
DP 3-2 Navigating physician education: Curriculum mapping gives new directions to continuing professional development courses

Jessie Thuswaldner University of Ottawa, Heather Lochnan University of Ottawa, Paul Hendry University of Ottawa, Robert Parson University of Ottawa

Background/Purpose: Family physicians must maintain competence over a broad range of topics to ensure quality care for their diverse populations. Continuing professional development (CPD) courses provide one avenue for family physicians to stay upto-date in their knowledge and skills. Curriculum mapping is a useful way to analyze the course offerings and determine to what extent the objectives align with the vast array of competencies required of family physicians. The map can be used by planning committees as part of their needs assessment process.

Methods: Using Microsoft Excel, we compiled a total of 547 individual presentations from the 36 different CPD courses that took place between 2017-2019. We mapped these courses to a list of competencies for family physicians developed by consensus of stakeholders. We assigned a single point to each time a competency was addressed in a presentation's learning objectives. Analysis of these results was done to inform future planning.

Results: Among the 547 presentations reviewed, competencies met most often were within the broad and overlapping categories of "Care of the Adults" (85.9\%) and "Family Medicine Expert" (90.4\%). Competencies met the least often were mapped to the categories of "End-of-life-care" (0.9\%) and "Maternity and Newborn care"(2.4\%). Within the nonmedical expert CanMEDs categories Health Advocate (4.9\%) and Professional (5.7\%) were lowest.

Conclusion: Curriculum mapping is a useful tool to identify gaps in competencies. This can be used as a guideline when designing learning objectives for future CPD courses. The results can also facilitate reflection on why some competencies are over or under-emphasized.
DP 3-3 Implementation of a Social Paediatrics Curriculum: Opportunities and Challenges to Cultivating Compassionate Care, Advocacy Skills and Health Equity Understanding

Jacqueline Ogilvie Western University, Breanna Chen Western University, Jill Sangha Children's Hospital, London Health Science Centre, Andrea Ens Western University

Background/Purpose: Understanding the impact of social determinants of health in paediatrics is essential to caring for children and in turn, to training in paediatric and primary care. Further, the attitudes with which physicians deliver care can be as therapeutically important as the medical treatment they provide (Markakis et al., 2000). While there is a lack of evidence for how to best teach such integral concepts, there is rising opinion that successful curricula development in this area will require a fundamental change in medical education (Sharma et al., 2018). We identified gaps in teaching Social Pediatrics in our pediatric residency program through faculty consultation, curriculum mapping and resident focus groups. Thematic analysis identified challenges and opportunities to learning social pediatrics related to personal/ learner factors and structural/systemic factors.

Summary of the Innovation: Funded through an AMS Phoenix Fellowship grant, we implemented a 3-year longitudinal social pediatrics curriculum that is woven within our paediatric residency training program. The curriculum is delivered through (1) academic teaching; (2) community experiences; (3) independent learning modules and (4) a hands-on Advocacy Project. Our curriculum aims to move beyond our traditional approach of teaching social determinants, towards cultivating competency in health advocacy and compassionate care.

Conclusion: Our curriculum has been implemented and resident feedback monitored. New approaches to physician culture, identity and bias are encouraged by this curriculum, which, through community engagement and experiential activities, challenges traditional physician-to-physician models of didactic teaching and learning. We outline the pedagogical 
principles that guided curriculum development, highlight key decisions made and challenges navigated to sustain change.

\section{DP 3-4 Curricular Innovations in}

Postgraduate Psychiatry Training: Lessons Learned from the 'One Room Schoolhouse' Pilot Program at McMaster University

Sarah Payne McMaster University, Sheila Harms McMaster University, Natasha Snelgrove McMaster University

Background/Purpose: The 'One Room Schoolhouse' (ORS) curriculum represents a radical shift in the model of teaching and learning for psychiatry residents. Residents identified that the current academic curriculum was most often taught in isolation, lacking integration with clinical training experiences. By reconstituting the learning group and incorporating a number of pedagogical innovations anchored to a clinical case, ORS attempted to address these concerns.

Summary of the Innovation: Six residents across all training levels voluntarily formed a learning group to participate in the pilot project. A lead resident was responsible for generating a complex clinical scenario, followed by group objective setting. Learning occurred over 3 iterative sessions as compared to stand-alone traditional academic sessions. Pedagogical techniques included the incorporation of test-enhanced learning, a flipped classroom approach, concept integration exercises, and experiential learning linked to community settings. Data was collected to measure levels of satisfaction and change in knowledge using pre-post testing. A focus group was conducted to gather narrative feedback. All residents indicated that they would participate in future sessions despite the increase in workload. A number of focus group themes emerged including the link between accountability and learner engagement and the importance of a safe learning environment.

Conclusion: There is preliminary evidence to guide postgraduate educators in building a successful academic curriculum through the work of the ORS, which engages novel pedagogical efforts and promotes a learning environment that fosters psychological safety, resident initiative and engagement, as well as formative assessments that facilitate reflective learning.

\section{DP 3-5 Creating an outcome-based curriculum map for a post-graduate medical education residency program}

Ryann Kwan Memorial University of Newfoundland, Roger Chafe Memorial University of Newfoundland

Background/Purpose: Currently, the Royal College of Physician and Surgeons of Canada (RCPSC) is in the process of rolling out its model of outcome-based medical education dubbed "Competence By Design" (CBD). As each residency program transitions to CBD, they must review their entire curricula to reflect new sets of competencies and incorporate novel assessment tools. This process involves re-structuring a complex curriculum framework, which can be aided by curriculum mapping. A description of how a residency program can go about mapping all of the RCPSC outcomes and competencies to create a blueprint that coordinates the characteristic residency learning opportunities and their corresponding ideal assessment methods would be a helpful guide in this challenging process. Thus far, no such process has been described in the literature. The purpose of this project was to create an outcomebased curriculum map of Memorial University's pediatrics residency program. The process used to create this map was then extrapolated to describe a general model to curriculum mapping an entire residency program to ensure compliance with outcome or competency-based medical education.

Methods: We systematically "mapped" the required RCSPC outcomes for our specialty to the learning opportunities available in our program. We first created individual maps for each of the clinical rotations in our residency program. The individual maps were cross-referenced and compiled to create a master program map using spreadsheet software. The master program map was then analysed for gaps and redundancies to inform a review of the curriculum as well as the creation and implementation of outcome-based assessment processes for each learning opportunity. Following completion of this mapping and curriculum review, 
our process was evaluated and generalized to create a model that other programs could follow.

Results: Through the creation and evaluation of our curriculum map, we were able to identify a large number of RCPSC outcomes that were missing from our curriculum. To address these gaps, we revamped our academic half-day curriculum to systematically address outcomes over the course of a 3-year rota, and created new curriculum components including required advocacy projects and quality improvement initiatives. We also developed assessment tools to specifically evaluate whether or not each learning opportunity fulfilled its intended outcomes. Finally, a ten-step general model for curriculum mapping a residency program was developed through evaluation of the process we used to create our program's map.

Conclusion: Curriculum mapping can be a powerful tool when used to evaluate the curriculum of an entire residency program, especially in this shifting environment of outcome- and competency-based medical education. We have created a simple tenstep model to evaluate a residency program through curriculum mapping. By using the model that we have presented, a residency program will be able to create a comprehensive outcome-based curriculum complete with assessment processes, which reflect all of the required learning outcomes and competencies expected of their residents upon completion of the program.

\section{DP 3-6 An evaluation of a pediatrics residency research curriculum}

Martha Balicki University of Manitoba, Barr Darja University of Manitoba, Robert Renaud University of Manitoba, Atul Sharma University of Manitoba, Celia Rodd University of Manitoba

Background/Purpose: In 2016, the University of Manitoba Pediatrics Residency launched a 4-week Academic Skills \& Knowledge (ASK) rotation for first year residents (PGY1). Topics included statistics, research design, and critical appraisal. The goal of this study was to evaluate ASK to assess the rotation's value and facilitate revisions.

Methods: In 2017, pre- and post-ASK focus groups assessed resident goals and feelings about the rotation. Written exams before (time0) and immediately after (time1) the rotation assessed knowledge gains (2016-2018) and retention (time2, 6mo post-ASK, 2017-2018). PGY1s in each ASK cohort (10-11/year) and 8 non-ASK residents (typically PGY4s) were compared with the same exam. Mean test scores were compared using paired and unpaired t-tests. Focus groups were transcribed and analyzed qualitatively for themes by two analysts.

Results: In the pre-ASK focus group, residents looked forward to ASK; goals centered around growing from learner to expert (in research, teaching and medicine) and developing skills in critical appraisal. Local research contacts and expert advice were also expected. Post-ASK, residents were very satisfied with the rotation overall. The written exam (maximum score $=36$ ) showed significantly increased knowledge (time0 mean $\pm S D=19.6 \pm 3.9$; time1=29.9 $\pm 3.2, p<0.001)$. Knowledge retention was intermediate (time $2=26.7 \pm 4.5$; comparing time 0 and time2, $p<0.001)$. Non-ASK residents $(22.7 \pm 7.1)$ had significantly lower scores than ASK residents at time1 $(p=0.02)$ but not time 2 .

Conclusion: Our study demonstrated participant satisfaction with ASK and increased knowledge that persisted over time in PGY1s, who matched the knowledge of more senior residents. Limitations include small sample size. Given its positive reception, ASK is now an essential rotation in the first half of PGY1.

\section{DP 3-7 Development of a Paediatric}

\section{Residency Professionalism Curriculum}

Lauren Friedman University of Toronto, Shannon Willmott University of Toronto, Marie-Pier Lirette University of Toronto, Larissa Shapka University of Toronto, Natalie Jewitt University of Toronto, Gabriel Tse University of Toronto, Amy Zipursky University of Toronto, Lisette Yorke University of Toronto, Adelle Atkinson University of Toronto, Michael Weinstein University of Toronto, Angela Punnett University of Toronto

Background/Purpose: Perceived lapses in professional behaviours are becoming increasingly common in residency programs. Research supports that skills in professionalism can be taught through 
role-modelling and structured curricula. To enhance professionalism in the Paediatric Residency Program at the Hospital for Sick Children, a novel curriculum was developed. The curriculum aims to cover content that is immediately relevant to residents, to facilitate recognition that expectations and practice culture may differ, and to incorporate evidence supporting professionalism training through reflection, mentorship, and small-group learning.

Summary of the Innovation: The curriculum is comprised of five case-based sessions. Each session focuses on a different professionalism issue (requesting time off, use of technology and social media, interprofessional interactions, patient care, and boundaries). These themes were generated from real-life examples of professionalism issues that were anonymously submitted by residents and faculty and then framed by the professionalism objectives outlined by the American Board of Pediatrics. The curriculum aims to bring residents, fellows, and faculty together to discuss perceived lapses in professionalism in order to generate open discussion, a shared understanding, and approaches for promoting professional behaviours moving forward.

Conclusion: Professionalism is an essential component of medicine and has been identified as a core competency in medical education. Our novel curriculum aims to enhance professionalism training by bringing staff and residents together to facilitate interactive, case-based professionalism discussions. The curriculum is generalizable across a variety of subspecialty programs and can be easily implemented. Participants endorsed open discussion involving staff and residents, cases that accurately reflected real life situations, and effective take-home points.

\section{DP 3-8 Audit of Radiology Knowledge Among Medical Students in a Problem- Based Learning Curriculum}

\author{
Senthujan Gunaseelan McMaster University, \\ Danielle Walker McMaster University, Natasha \\ Larocque McMaster University
}

Background/Purpose: Until recently, no formal undergraduate radiology curriculum has existed at McMaster University. We performed an audit of the radiology knowledge of the last graduating class who received no formal radiology teaching. This data will serve as a baseline to assess the longitudinal impact of the new curriculum we are developing.

Methods: A 50-question multiple-choice quiz was administered to graduating medical students covering the topics of radiologic anatomy, imaging interpretation and appropriate imaging ordering. The validated questions were obtained through an international database provided by the Alliance of Medical Student Educators in Radiology (AMSER). The web-based platform used to create/administer the quiz (Radiology ExamWeb) provides detailed statistical analysis on each question; as such, the scores of McMaster students could be compared to international results.

Results: A total of 172 (83.5\%) McMaster students completed the quiz. The mean score for McMaster students was $64.5 \%$ vs. $72.8 \%$ (international average) $(p=0.02)$. McMaster students had lower scores on radiologic anatomy (61.0\% vs. $79.1 \% ; p=0.01)$ and imaging interpretation ( $51.7 \%$ vs. $67.4 \%$; $p=0.04)$ sections. There was no difference between the groups on the questions involving appropriate imaging ordering (73.8\% vs. $71.8 \% ; p=0.71)$.

Conclusion: This study noted deficiencies in the knowledge of McMaster graduates in the areas of radiologic anatomy and image interpretation. Our new curricular initiatives will focus on these areas with a goal to improve McMaster students' quiz results. 
DP 3-9 Reviewing non-cancer pain \& opioid prescribing curriculum using INSPQ competencies

Michelle Gibson Queen's University, Portia Tang Queen's University

Background/Purpose: In view of Canada's opioid crisis, we reviewed the undergraduate curriculum at our institution to determine if it reflects current attitudes, beliefs \& knowledge in opioid prescribing \& related topics.

Methods: Learning events within the 'Pain' \& 'Substance Abuse' integrated threads were extracted $\&$ analyzed from our learning management system. The content in each learning event was summarized \& compared to medical competencies published by the Institut National De Santé Publique Du Québec (INSPQ) in non-cancer pain management \& opioid prescribing.

Results: Twenty-six learning events related to 'Pain' \& 12 learning events related to 'Substance Abuse' were identified. The INSPQ describes 4 clusters of competencies: 1. Basic epidemiological, clinical \& pharmacological knowledge relevant to non-cancer pain \& opioids, 2. Evaluation of pain intensity \& consequences of pain, 3. Evaluation of substance abuse \& misuse, \& 4. Management \& follow up in patients using opioid therapy. Most (6/7) competencies within the first cluster were covered in the curriculum. Almost half (2/5) of competencies related to the second cluster were not explicitly covered, \& 3/7 of competencies related to the third cluster were not covered. A third (3/9) of competencies within the fourth cluster were not explicitly covered.

Conclusion: Our curriculum addresses competencies needed to manage non-cancer pain \& prescribe opioids. This initial review identified potential gaps in other core competencies pertaining to opioids \& related topics, such as substance abuse. Limitations to our review are that we could not analyze untagged learning events or new sessions. Next steps will include consultation with stakeholders to determine the extent of gaps in the curriculum and how they can be filled.
DP 3-10 Improving Medical Education Through Community Service-Learning: Assessing the needs of community organizations involved in service-learning

Ramy Boles University of Toronto, Leshawn Benedict University of Toronto, Joyce Lui McMaster University, Roxanne Wright University of Toronto, Fok Han Leung University of Toronto

Background/Purpose: While literature exists on student feedback to community service-learning (CSL), community organizations have played a small role in re-shaping CSL within medical curricula. This study aimed to analyze feedback from community supervisors involved in a CSL course at University of Toronto's Faculty of Medicine.

Methods: Semi-structured interviews with supervisors $(n=9)$ were conducted between October 2018 - January 2019. Interviews were transcribed and analyzed through thematic analysis.

Results: Four overarching themes were identified. Perceived benefits to students by supervisors focused on their contributions to the development of future physicians through understanding community needs and exposure to organizations that may be resourceful to physicians. Secondly, organizational benefits of CSL were discussed including having extra hands to assist, work-specific benefits, and medical student's unique expertise. CSL benefits were not without challenges. Scheduling difficulties, motivating students to understand their work's value, and curriculum gaps identified were challenges supervisors faced. Three subthemes emerged under areas for improvement. Supervisors emphasized increasing flexibility to make experiences more meaningful. They also wanted more dialogue between organizations to gain insight from one another and a clearer understanding of CSL and its role in a curriculum.

Conclusion: Community supervisors viewed academic institutions to be in a unique position having established many partnerships with community organizations. Developing networking opportunities between organizations should be encouraged across CSL programs in medical education. As well, clearer understanding of CSL as 
part of the medical curriculum was important for supervisors to better understand their roles and responsibilities.

\section{DP 4-1 The novel use of virtual patient cases with expert traces for rare disease education in hematology}

Zachary Liederman University of Toronto, Ivan Silver University of Toronto, Rita Selby University of Toronto

Background/Purpose: Within subspecialty residency programs there is a need to develop new training mechanisms for rare gray zone disorders (i.e. disorders where there is no consensus). Trainees must demonstrate competency in these areas but limited exposure and the absence of clear guidelines, makes learning increasingly difficult. The use of virtual patient cases is one approach to provide learners concrete experience for these rare disorders. Furthermore, when presented alongside expert testimonials or traces (described by Posel N. 2009), which delineate the spectrum of acceptable management choices, learners can develop sophisticated approaches to clinical ambiguity. This pilot study explores the use of VIRTUAL patient Cases with Expert Traces (Virtual CasET) for the teaching of rare gray zone disorders.

Summary of the Innovation: Two virtual patient cases on rare gray zone thrombotic disorders were created through professional consensus using the VIC editor. Thrombosis experts $(n=7)$ completed the cases and traces were generated based on their decisions (e.g. treatment choices). Hematology trainees $(n=12)$ subsequently completed the cases and were shown the expert traces. Learners contrasted their approach with the expert traces and participated in a guided case debrief. Learners completed a questionnaire which included pre/post ratings of clinical confidence. Qualitative thematic analysis of the debrief was performed using phenomenological inquiry.

Conclusion: Virtual CasET is a promising approach to rare gray zone conditions. Learners positively rated their experience and noted improved confidence in handling uncertainty. Learners were able to identify and integrate core principles from the traces. Unexpectantly, learners endorsed an enhanced appreciation for collaboration with colleagues when faced with gray zone cases.

\section{DP 4-2 Clinical teaching unit design: A} realist systematic review of evidence-based practices for clinical education and health service delivery

Ryan Sandarage University of British Columbia, Brandon Tang University of British Columbia, Katrina Dutkiewicz University of British Columbia, Stephan Saad University of British Columbia, Jocelyn Chai University of British Columbia, Kristin Dawson University of British Columbia, Vanessa Kitchin University of British Columbia, Rose Hatala University of British Columbia, lain McCormick University of British Columbia, Barry Kassen University of British Columbia

Background/Purpose: The Clinical Teaching Unit (CTU) has emerged as a ubiquitous model of clinical education across medical schools, since its inception over 50 years ago. However, health care has changed dramatically over this period, and we thus reviewed existing evidence on principles of CTU design that optimize clinical education and health service delivery in the 21st century.

Methods: We performed a systematic review in accordance with the Cochrane Review protocol. Databases, including MEDLINE, Embase, and Cochrane Database of Systematic Reviews, and CINAHL, were searched to find primary research articles published from 1993 to 2019 which discussed trainee education and/or health care delivery in the context of a clinical teaching unit or other teaching ward.

Results: Studies meeting inclusion criteria after fulltext review were analyzed according to the Star Model viewing health systems as a collection of interdependent subsystems including: strategy, structure, human resources, incentives, and information and decision support. We added a sixth subsystem for education given its core relevance to CTUs. Most existing evidence focuses on the structure and education subsystems, including approaches to ward rounds, optimal call structures, and novel educational strategies. However, many articles involved low to moderate quality evidence. 
Conclusion: Efforts should be made to generate higher-quality evidence on the design of clinical teaching units as a dual model for both clinical education and health service delivery. Further knowledge translation efforts may be necessary to ensure that known best practices in CTU design become common practice.

\section{DP 4-3 Development of a mobile application} to increase motivation, engagement and teaching activity of clinical faculty using gamification principles.

Aazad Abbas University of Toronto, Sarah McClennan University of Toronto

Background/Purpose: Medical education is a fundamental principle of an academic healthcare institution. However, compared to research and patient care roles, clinician teachers have historically felt undervalued and underappreciated. Research has shown that clinician teachers are intrinsically motivated for their work, but there is a lack of institutional recognition for their contributions to medical education (A. Freeland, 2019). In our large community hospital with an academic mandate, we propose to recognize and reward the efforts of clinician teachers using a mobile application based on gamification principles.

Summary of the Innovation: We developed a mobile application named TutorTracker using the cross platform (i.e. Android, iOS, Windows etc.) and opensource development software Ionic. Teaching data is extracted from the education office spreadsheets and downloaded to the application. Using a password protected application, physicians can track their clinical teaching using various parameters such as hours of online teaching, date, subject and course. The application then rewards users, using virtual badges, for quantity and diversity of teaching. The application also provides a summary of the users clinical teaching history for ease of submitting yearly hospital and university renewals as well as for documenting yearly continuing medical education (CME).

Conclusion: Gamification principles have been effectively implemented to construct a mobile application allowing clinician teachers to track and be rewarded for their educational responsibilities. Next steps would be to evaluate the application utilization and satisfaction, assess change in physician teaching and to iterate improvements in the application.

\section{DP 4-4 Medical Student Comfort Level with}

the Neurological and Musculoskeletal

\section{Examinations}

Wasimuddin Syed Queen's University, Syed Ibrahim Queen's University, Ali Dergham Queen's University, Laura Milne Queen's University, Jessica Trier Queen's University

Background/Purpose: Arthritis, diabetes, back pain and headache are common reasons for medical visits. These presentations require a combination of neurological and musculoskeletal physical examinations, highlighting the importance of proficiency in them. However, it is unclear how comfortable medical students are with these examinations as they enter clerkship. The aim of this study is to examine pre-clerkship medical students' comfort level with performing and interpreting the neurological and musculoskeletal examinations.

Methods: This cross-sectional study surveyed preclerkship medical students at Queen's University School of Medicine. Students completed a web-based electronic survey with questions about demographics, studying resources, practice patterns, comfort with neurological and musculoskeletal examinations, and curricular feedback. Data analysis was performed using Microsoft Excel and IBM SPSS.

Results: 90 students (48.9\% Year 1, 51.1\% Year 2) participated in this study. $15.6 \%$ of students were uncomfortable with both the neurological and musculoskeletal examinations. Within the neurological exam, students were least comfortable with cognitive testing (63.3\%) and sensory examination (14.4\%). Within the musculoskeletal exam, students were least comfortable with wrist (75.5\%) and elbow (70.0\%) exams. Students provides 24 unique feedback suggestions for improvement in the clinical skills curriculum.

Conclusion: This study highlighted specific components of the neurological and musculoskeletal examinations that pre-clerkship medical students were least comfortable performing. Feedback from 
this survey will be used to improve the clinical skills curriculum and course manual, including opportunities for more frequent supervised practice and periodic review.

\section{DP 4-5 Pre-Clerkship Procedural Skills Pilot Program}

Patrick Albers University of Alberta, Brodie Ritchie University of Alberta, Alexander Miles University of Alberta, Eleanor Crawford University of Alberta

Background/Purpose: An identifiable area for improvement in Canadian medical school curriculum is the decline in safe learning opportunities for students to perform clinical skills. As a result, anxiety around performing clinical procedures is increasing, while confidence and self-perceived knowledge of clinical skills is decreasing. Providing low-risk learning opportunities for pre-clerkship medical students to practice essential clinical skills would help to address this learning gap.

Summary of the Innovation: A program modelled on a pilot study by Battaglia et al. was implemented at the University of Alberta. Six second year students were selected as peer teachers and trained in how to teach four high-yield clinical skills by residents. These students then taught 18 second year medical students using a near-peer teaching model to reduce anxiety and expectations regarding performance. Self-perceived knowledge and mental status were examined before and after the four sessions. There was a significant ( $\alpha=0.05 ; p=0.0008$ ) difference in mean state anxiety scores pre and post program using a State-Trait Anxiety Inventory. All four sessions resulted in significant increase in self-perceived knowledge, including suturing, airway management, IV starts, and local anaesthetic $(\alpha=0.05 ; p=0.0007$, $0.0000,0.0001 .0 .0001$, respectively).

Conclusion: Implementing a near-peer pre-clerkship clinical skills program resulted in a decrease in situational anxiety and an increase self-perceived knowledge of clinical skills. This study contributed toward the integration of clinical skills for preclerkship students at the University of Alberta, but further studies are recommended to continue to develop clinical curriculum.

\section{DP 4-6 A 'systems thinking' conceptual framework to explore clinical practice.}

Alan Batt Monash University, Australia, Brett Williams Monash University, Madison Brydges McMaster University, Matthew Leyenaar McMaster University, Walter Tavares University of Toronto

Background/Purpose: The nature of clinical practice is ever-changing, varies according to the attributes of those enacting it, and poses complex questions that must be considered within context. As such, healthcare professionals need the ability to understand and respond to the larger contexts of the healthcare system. Existing approaches to describing clinical practice may be limited in how they conceptualize such context and complexity.

Methods: We sought to evaluate how conceptualization of paramedic practice may be optimized using a systems approach. We examine the role of combining an adaptation of Ecological Systems Theory (EST) and complexity thinking as ways of describing paramedic practice.

Results: EST can be adapted to outline the elements of clinical practice. This obligates a focus on various healthcare system levels which are often missing from our descriptions of practice. Complexity thinking obligates a focus on the non-linear, unpredictable nature of relationships between these elements. When combined, these frameworks allow for identification of elements within the system that may have value in describing clinical practice. We illustrate how aspects of paramedic practice may be ignored until viewed from a merging of these approaches.

Conclusion: Merging EST with complexity thinking provides an opportunity to reflect the complex realities of clinical practice when attempting to describe it. This conceptual framework promotes a focus on factors from macro level to individual patient level, as well as on the relationships within the system. This can be used as a foundation to inform future efforts to represent clinical practice, such as in the development of competency frameworks. 
DP 5-1 Development of a Canadian Program of Continuing Professional Development for Emergency Nurses

Mélanie Marceau Université de Sherbrooke, Denis Bouchard Society of Professionals in Emergency Care (SoPEC), Monique McLaughlin Society of Professionals in Emergency Care (SoPEC), Brian Lee Society of Professionals in Emergency Care (SoPEC), Landon James Society of Professionals in Emergency Care (SoPEC)

Background/Purpose: Continuing professional development (CPD) is an essential component of nurses' practice, especially in Emergency Departments, which are characterized by constantly evolving practices and technologies. Nevertheless, there are no current CPD programs in Emergency care for Canadian nurses. The purpose of the project was to develop a forward-thinking program that would encompass the Emergency Nurses' core competencies using evolving research-based on adult learning principles.

Summary of the Innovation: A team composed of nurses from Québec and British Columbia have led the development of a bilingual education program with the collaboration of clinical experts across Canada. Peer review ensured the quality of the content and translation in both languages (English and French). Emergency Practice, Interventions and Care - Canada (EPICC) program includes four courses lasting one or two days: 1) emergency care, 2) trauma care, 3) pediatric care and 4) educator. All courses are based on active learning and promote feedback to drive learning. The EPICC program focuses on 1) the ability to assess a situation, 2) interpret it, 3) communicate, and 4) react and seek help as needed.

Conclusion: Since 2016, 3525 learners have participated in one or more EPICC courses across Canada. EPICC uses innovative ways to promote education for emergency nurses. Challenges include standardization of teaching and implementation in rural areas. Data collected shows a high level of satisfaction from participants. Future research is planned to measure the impact of the program such as the improvement of team communication.
DP 5-2 Evaluation of a Summer Healthcare Improvement Program (SHIP) in Undergraduate Medicine Education

Paul Barber University of Alberta, Jennifer Halasz University of Alberta, Leslie Truong University of Alberta, Kendra Raffael University of Alberta, Christine Phan University of Alberta, Pam Mathura University of Alberta, Narmin Kassam University of Alberta, Tracey Hillier University of Alberta, Jennifer Croden University of Alberta

Background/Purpose: Quality Improvement (QI) science is quickly becoming an effective and acceptable approach for teaching physicians how to inspire and lead positive change in healthcare. Currently, across Canada there is minimal experiential education for undergraduate medical students regarding QI. Students learn little about how they can change the systems that they will work in and are missing out on exposure to the leadership potential of their roles. In a response to this opportunity, the Department of Medicine, Alberta Health Services and Undergraduate Medical Education office developed the Summer Healthcare Improvement Program.

Summary of the Innovation: To provide students with hands-on QI experience, a 15-week program was launched in 2017. Since then, 19 students have completed 22 projects. Students work closely with a Sr. Quality Consultant and a MD to gain on-theground experience running projects. Additionally, students received optional QI readings, and opportunities to attend quality council meetings and regional QI days. All students also received Evidencebased Practice in Quality (EPIQ) certification. To assess the effectiveness of the SHIP program, a pre and post-survey was implemented along with a SWOT analysis.

Conclusion: $90 \%$ of students who responded to the surveys thought their peers could benefit from participating in SHIP and $71 \%$ of the respondents thought their SHIP experiences would be useful in future practice. Difficulties included gaining funding, coordinating with student schedules and ensuring students saw all parts of the QI process. Overall, students expressed a desire to learn more about QI and were engaged when given the opportunity to 
learn more. It was felt that SHIP demonstrated the need for and benefit of $\mathrm{Q}$ l education earlier in medicine.

\section{DP 5-3 A unified assessment evaluation framework for educational quality improvement}

Shujiao Wang McGill, Maryam Wagner McGill, Valérie Dory, Patricia Wade McGill, Beth-Ann Cummings McGill

Background/Purpose: Quality improvement is critical in improving institutions' practices, priorities and performances. In undergraduate medical education (UGME) programs an accreditation requirement, and one of the key quality improvement initiatives, is the review of curricular and assessment. However, there are multiple types of courses with varying objectives, structures, and modes of delivery. This variability introduces numerous challenges to identify a single evaluation approach, particularly in assessment. Whilst some assessment frameworks exist in medical education, they $\bullet$ prioritize only one essential aspect (e.g., validity), but neglect others $\bullet$ lack for flexibility of evaluating different types of assessments $\bullet$ focus on outcomes but neglect the process of evaluation

Summary of the Innovation: • A unified framework is developed to evaluate assessment use and interpretation of outcomes that is applicable across all of UGME courses. This framework is theory-based, research-supported, and practice-oriented. It not only addresses the key criteria of assessment, but also fosters a culture of continuous improvement by aligning with CACMS Accreditation Standards; • Multiple data sources generated from this framework taking into account all relevant stakeholders paint a rich, multifaceted picture of the assessments; • The implementation of this framework at both classroom and clinical-based courses effectively identifies the strengths and challenges. It contributes to professional development by raising awareness and broadening skills for designing assessments for educators.

Conclusion: This innovative framework of assessments evaluation not only integrates key features and accreditation standards of assessment, but also serves as a unified methodological guide of course review in any institution to achieve the quality improvement goals.

\section{DP 5-4 Speaker effectiveness in continuing medical education: a systematic review}

Hannah Smith University of Manitoba, Diana Sanchez-Ramirez University of Manitoba

Background/Purpose: Speakers play a key role in making Continuing Medical Education (CME) programs effective for participants. While evidence has identified distinctive characteristics of an effective lecture, to the best of our knowledge, there is no clear definition of "speaker effectiveness" in CME. Objective: To compile existing evidence related to speaker effectiveness in CME programs.

Methods: Systematic review of the literature studying CME participants' perspectives of speaker effectiveness, filtered by full-text and the English language.

Results: Seventeen articles met the inclusion criteria and were selected for full text review. Results suggested that speakers who incorporated ARS/active learning/interactive learning, case studies/clinical cases and specific (rather than broad) program content, were generally deemed to be more effective. With respect to presentation layout/articulation, speakers who utilized workshops, kept presentations shorter in duration and spoke coherently, were also considered to be more effective. Mixed results were seen regarding speakers' academic rank/credentials and the impact this has on speaker effectiveness. Perceptions of speaker effectiveness were also audience-specific, varying on age, stage in education and specialty.

Conclusion: While the literature provides some characteristics of "speaker effectiveness", further studies are needed to clarify this concept in the context of CME. Results of this study might guide speakers committed to improve the effectiveness of their future CME presentations. 
DP 5-5 Initial evaluation of a new rural mentorship program for preclinical medical students

Jasmine Waslowski University of Toronto, Morag Paton University of Toronto, Mary Freymond University of Toronto, Tristan Brownrigg University of Toronto, Sagar Patel University of Toronto, Shelby Olesovsky University of Toronto, Joyce Nyhof-Young University of Toronto

Background/Purpose: Rural clinical exposure plays a vital role in recruiting and retaining physicians to rural areas, while structured mentorship programs support student development. We describe the structure and evaluation of a new Rural Mentorship Program (RMP), which provides pre-clinical medical students at our large urban medical school with rural exposure through mentorship. We aim to identify benefits and challenges of the RMP, assess how mentorship from rural physicians influences medical student perceptions and intentions towards rural careers, and identify possible facilitators of success.

Summary of the Innovation: The RMP paired 1st and 2nd year medical students with physician mentors in four rural communities. In response to a relative deficit in rural exposure at our institution, this student-developed program was delivered as a 3month pilot. During the program, mentees communicated with mentors remotely and visited their mentor's community for at least one clinical experience. Mentor and mentee questionnaires assessed program satisfaction and attainment of objectives. Mentees rated their perceptions and intentions for rural careers pre- and postparticipation. Focus groups and interviews were used to deepen insights. Though there was no statistically significant impact on career intentions, participants reported overall satisfaction. The early rural clinical exposure was believed to be the most valuable component. Mentees appreciated the authentic relationship with their mentors, and mentors valued the enriching teaching opportunity.

Conclusion: Our results will contribute to literature supporting the role of urban medical schools in advancing rural medical education and promoting recruitment of rural physicians. Further program implementation is required to observe possible longterm impact.

\section{DP 5-6 Experiences from a new Women in Medicine club}

Adrina Zhong Western University, Elise Quint Western University

Background/Purpose: While women account for more than half of graduating medical students, women physicians are outnumbered in leadership roles, more likely to experience mistreatment or harassment, and report higher burnout rates. In an effort to mitigate gender bias in medicine and promote well-being in women medical trainees, a Women in Medicine student club was established to create a safe space to discuss unique issues that affect women in medicine, share resources and provide support.

Summary of the Innovation: Events held by the club included informal discussion groups, several lunchtime talks, and a week of events surrounding International Women's Day. The club worked together to provide recommendations to change student mistreatment policies. An active Facebook group where club members post news and event links contributed to the development of a sense of community. The inaugural year of the club culminated in a mentorship event which brought together female physicians and medical students in a speed networking format. Anonymous qualitative feedback was solicited at the end of the event.

Conclusion: The mentorship event was met with excellent feedback from both physicians and students. Physicians enjoyed meeting students and providing career advice. Some physicians reflected that they wished there was a similar initiative in place when they were in medical school. Students appreciated insights from a variety of medical specialties, especially as it pertains to career exploration. Students and physicians alike praised the relaxed nature of the mentorship event held at a local coffee shop without the demand of clinical duties. Next steps for this new club include continuing the mentorship event in upcoming years as well as continuing to develop community and support among women medical students. 
DP 6-1 Sink-or-Swim No More: Development and Implementation of a Shadow Call During Orientation for Incoming Pediatric PGY1 Residents

Tobey Audcent University of Ottawa, Jimin Lee University of Ottawa

Background/Purpose: There is a marked increase in clinical responsibilities on call for pediatric residents during Transition to Discipline. Following a survey of the outgoing pediatric PGY1 residents, we identified a need to provide a more structured and experiential orientation for the incoming PGY1 residents prior to their first night on call. We created a "shadow call" to orient the incoming PGY1 residents, increase their level of comfort on call, and optimize patient safety.

Summary of the Innovation: In July 2019, pediatric PGY1 residents were paired with PGY2 residents for a "shadow call". Objectives, structured checklist, observation notes and discussion prompts were developed through a needs assessment survey of the outgoing PGY1 residents. The PGY1 residents followed a PGY2 resident during handover, an admission, assessment of ward patients and documentation. Discussions were encouraged. Participant satisfaction was evaluated by a voluntary online survey.

Conclusion: Twelve out of twelve incoming PGY1 resident participants felt that the shadow call was a valuable component of the orientation, and identified one-on-one discussion with a PGY2 resident to be the most useful aspect. They reported an understanding of the expectations on call, comfort with their first night on call, and confidence in their ability to care for patients safely. All five PGY2 residents reported that having a shadow buddy did not add to their workload on call, and noted the benefit of building a meaningful relationship with the PGY1 residents. While the shadow call was designed to be observational, some PGY1 residents identified areas where greater participation under direct supervision of a senior resident would have further enhanced the experience.
DP 6-2 Food as medicine: How can we teach it?

Andrea Guerin Queen's University, Victoria Lee-Kim Queen's University, Karen Grewal Queen's University, Shannon Willmott University of Toronto, Theresa Nowlan-Suart Queen's University

Background/Purpose: Many physicians lack confidence in their knowledge of nutrition stemming from inadequate instruction in training. At Queen's medical school, nutrition is assigned to the first year Pediatrics course, tied to childhood growth and development. Recognizing its position early in the curriculum provides a unique opportunity, we asked: Can nutrition concepts be constructed and delivered in first year medical school in a way that allows for retained knowledge and confidence in clerkship?

Summary of the Innovation: A backward design model was applied to create a cohesive nutrition unit. A survey was developed to provide evidence as to the efficacy of the program, capturing knowledge and comfort with the material. Varied learning events with a clear progression of complexity were designed. The survey was administered to the students both before and after the unit and compared to their peers in clerkship. The survey was then re-administered to the students who participated in the unit in clerkship to address retention of knowledge and confidence. There was no difference between the first-year students and clerks prior to implementation. However, first-year students performed significantly higher and felt more confident after the unit $(p<.05)$. Follow-up of the first-year cohort in clerkship revealed a significantly higher average score $(p=0.019)$ than the original clerkship group.

Conclusion: Creation of a cohesive nutrition unit in the first year Pediatrics course resulted in better retained knowledge and confidence for students in clerkship. Further study to address extension of this material is needed. 


\section{DP 6-3 A situational analysis of pediatric ophthalmology training needs in Ethiopia}

Sylvie Bowden University of Toronto, Helen Dimaras The Hospital for Sick Children, Asim Ali The Hospital for Sick Children, Sadik Taju The Hospital for Sick Children, Stephanie Kletke The Hospital for Sick Children

Background/Purpose: Postgraduate medical training at Addis Ababa University (AAU) has been enhanced through support from the University of Toronto ( $U$ of T) through the Toronto Addis Ababa Academic Collaboration (TAAAC). There is present need for improved training to address childhood blindness in Ethiopia. Our aim was to assess the feasibility of implementing a pediatric ophthalmology fellowship at AAU, supported by $U$ of $T$, modeled on the TAAAC.

Methods: A situational analysis was conducted at Menelik II, the teaching hospital providing ophthalmology services in Addis Ababa. Staff expertise and clinical, patient, and educational resources were reviewed and compared to International Council of Ophthalmology fellowship guidelines. Local training needs were evaluated. A strategic working meeting facilitated program specification.

Results: A two-year fellowship incorporating research, faculty feedback, case log review, and formal examination as quality indicators was proposed. Anterior segment, retinoblastoma, strabismus, and retinopathy of prematurity were identified as priority areas requiring support. Learning outcomes were deemed feasible given the high volume of complex cases, qualified staff supervision, and sufficient equipment. Telemedicine, development of a larger eye hospital, and partnerships to support equipment acquisition and maintenance were identified as strategies to manage barriers.

Conclusion: The on-site needs assessment demonstrated feasibility in implementing a pediatric ophthalmology fellowship, the first of its kind in Eastern Africa, and provided a way forward for its development. However, strategic partnerships may be crucial to ensure resource sustainability. Academic partnership between Canada and Ethiopia is an exciting avenue for initiatives to strengthen cultural competencies in both nations.

\section{DP 6-4 Collaborative initiation of the first} paediatric ophthalmology fellowship in

\section{Eastern Africa}

Sylvie Bowden University of Toronto, Helen Dimaras University of Toronto, Max Solish University of Toronto

Background/Purpose: Postgraduate medical training at Addis Ababa University (AAU) has been enhanced through support from the University of Toronto and the Toronto Addis Ababa Academic Collaboration (TAAAC). There was a clear need for improved training to address the high burden of visual impairments among children in Ethiopia. A situational analysis conducted at Menelik II, AAU's teaching hospital, demonstrated feasibility in implementing a pediatric ophthalmology fellowship, the first in Eastern Africa. Our aim was to develop curriculum following International Council of Ophthalmology's guidelines, modelled on the TAAAC.

Summary of the Innovation: A two-year fellowship incorporating research, faculty feedback, and formal examination as quality indicators was proposed. Anterior segment, retinoblastoma, strabismus, and retinopathy of prematurity were identified as priority areas requiring support. Modules were designed to have in-person training by SickKids Ophthalmologists, supplemented by online e-tutorials. A secure platform for online content was created to house module content, journal libraries, and equipment tutorials. A strong tele-education link between Menelik II and SickKids was developed. Additionally, pediatric-specific ophthalmology equipment was purchased.

Conclusion: Tele-education through successful transmission of lectures for fellows at SickKids to Ethiopia allowed real-time teaching. Lecture recordings were then put online for review as additional resources. The online component allowed trainees to personalize learning needs. Longevity in practice is supported by the online format, in-person training to build capacity, and provision of equipment difficult to source locally, with education in its care and maintenance. Continuous feedback from all 
stakeholders was incorporated throughout program development.

\section{DP 6-5 A multimedia approach to enrich} learning of developmental milestones in 2nd year medical students

Alanna Roberts Memorial University of Newfoundland, Samantha Stasiuk University of British Columbia, Alasdair Nazerali Maitland University of British Columbia

Background/Purpose: Given the numerous developmental milestones tracking the growth of infants and children, it is a daunting task for learners new to the topic. We designed a new multimedia resource to supplement this learning for 2 nd year medical students within the clinical skills curriculum.

Summary of the Innovation: The framework for the content was designed using key components of cognitive learning theory and principles of multimedia instruction. Based on the dual channel theory, it was decided to create multimedia content, optimizing both visual and verbal information processing. Given the extensive details within this topic and knowledge of cognitive load, attention was drawn to an appropriate level of detail while avoiding redundancy. We minimized extraneous processing by utilizing basic black and white drawings with accompanying text and key headings to highlight content. To manage essential processing, learners were encouraged to review a corresponding resource to optimize the learning before and during engagement with the video. We strived to foster generative cognitive processing using a recorded conversational style narrative. The multimedia content itself was created with VideoScribe (Sparkol, Bristol UK) using hand drawn illustrations that highlighted the slight nuances in child development.

Conclusion: This resource was developed in a 4 week research block by a 4 th year medical student in order to integrate educational theory and curriculum delivery. It was well received by reviewers, faculty and students and has become part of our core curricular content at UBC. We believe the novelty and feasibility of this innovation highlights the capacity we have to develop resources for learners.

\section{DP 6-6 Barriers to Breastfeeding in Physician and Trainee Moms}

Alex Frolkis University of Calgary, Allison Michaud University of Calgary, Shannon Ruzycki University of Calgary

Background/Purpose: The benefits of breastfeeding for both the mother and baby are well known. In North America, the proportion of female trainees and physicians in practice is rising. Medical students and residents train during the peak of their reproductive years, but breastfeeding rates tend to be lower in female medical professionals compared to the general population. The purpose of this study was to explore barriers to breastfeeding among female trainees and physicians.

Methods: We conducted a scoping review using Medline and Embase. Abstracts were considered for further review if they included information on breastfeeding in physician and medical student mothers. After the initial screen reference lists of included studies were reviewed for further study identification. Two independent reviewers read and extracted demographic information, barriers, and breastfeeding rates from included studies.

Results: A total of 2128 abstracts were screened and 55 studies fully reviewed. The most commonly identified barriers to maintaining breastfeeding were short length of maternity leave, heavy workplace demands without established breaks, perceived or actual lack of colleague support, and lack of appropriate pumping location or equipment. Women in hospitals that included dedicated pumping spaces with computer, telephone, and refrigerator access reported longer duration and better quality of continued breastfeeding experience.

Conclusion: Female trainees and physicians have many barriers to maintaining breastfeeding upon return to work. Departmental policies that include established breaks and conveniently located pumping locations with electronic medical record access and telephone might improve rates and achievement of breastfeeding goals. 
DP 6-7 Impact of stress on the performance of neonatal endotracheal intubation: a randomized controlled trial on manikins.

Ahmed Moussa Université de Montréal, Evelyne Wassef Université de Montréal, Thomas Pennaforte Université de Montréal, Michael-Andrew Assaad Université de Montréal

Background/Purpose: Although a required skill in pediatric residency programs, success rate of neonatal endotracheal intubation (NETI) among trainees is low. NETI is taught using simulation with limited short-term retention of skill. In the clinical setting, NETI is associated with stress, which effect on success is poorly studied. The objective is to assess the impact of stress on the success of simulated NETI.

Methods: Crossover randomized controlled trial. Pediatric residents randomized to perform NETI in low (LS) or high stress (HS) environment, and then crossover. Stress was created using audio alarms, third party supervisors and simulated manikin instability. Stress of participants was assessed at rest, before and after each scenario. Stress assessment included: salivary cortisol, heart rate and the State Anxiety Inventory (STAI) questionnaire. Primary and secondary outcomes include: success rate, time to successful intubation and stress levels. Success rate was analyzed using chi2 and continuous variables were analyzed using paired t-test and repeated measures ANOVA.

Results: 49 residents (97 NETI) have been recruited and have completed the study. Success rate in the HS group was $60 \%$ vs $51 \%$ in LS $(p=0.41)$. There was no significant difference with regards to duration of $\mathrm{NETI}$, duration of scenario or salivary cortisol. Heart rate was significantly different between $\mathrm{HS}$ and LS groups $(p<0.001)$. The STAI result was also significantly different between HS and LS groups $(p=0.01)$.

Conclusion: TAmong stress markers, the heart rate and STAI increase significantly more in the HS setting. The stable cortisol level suggest that stress lived by the resident does not create a sense of loss of control or distress. These findings suggest that the HS setting is an appropriate training tool for stress preparedness for neonatal ETI.
DP 6-8 A call to action: Development and Implementation of an Undergraduate Social Paediatric Curriculum

Andrea Ens Western University, Jacqueline Ogilvie Western University, Breanna Chen Western University, Erin Peebles Western University, Tara Mullowney Western University

Background/Purpose: Despite data supporting its staggering importance in health outcomes, medical curricula has not traditionally focused on social determinants of health (SDH). Commonly, curricula have positioned $\mathrm{SDH}$ as a list of risk factors to memorize rather than focusing on the upstream, broad societal factors that perpetuate disparity. Children are particularly vulnerable as adverse childhood experiences (ACEs) alter lifelong health trajectories. A 2019 scoping review reported the need for formal programming addressing SDH. Subsequently an expert consensus panel has put forward a call to action.

Summary of the Innovation: The objective was to create and implement a deliberate Social Paediatric curriculum prompting students to evaluate their own beliefs and bias, and equipping students with skills to identify actionable change. A review of the current undergraduate curriculum revealed a paucity of dedicated opportunity for addressing $\mathrm{SDH}$ in Paediatrics. This was particularly clear to see within the clerkship program, where concepts were integrated but not emphasized. Using consensus panel recommendations a longitudinal program extending from pre-clinical to clerkship years was developed. The curriculum involves: 1) interactive introductory online learning modules; 2) didactic teaching session in clerkship; 3) written reflection on a clinical case; 4) small group, inter-disciplinary casebased learning; 5) practice OSCE station.

Results: With early childhood development being particularly susceptible to the impact of SDH and health inequity, Paediatricians and Paediatric training has an obligation to rethink what is taught and how we can shift from knowledge and lists to action and integration. Next steps will include evaluation and revision of the program. 
DP 7-1 What's the Problem? Collaborating Across Specialties to Develop a Novel PGY1 Medical Psychiatry Case-Based Curriculum

Natasha Snelgrove McMaster University, Sandra Westcott McMaster University, Sheila Harms McMaster University, Kaif Pardhan McMaster University, Alim Pardhan McMaster University, Michael Brown McMaster University

Background/Purpose: Until 2019, PGY-1 psychiatry residents at McMaster University participated in academic learning sessions that were held with numerous other specialties, leaving psychiatry residents feeling that these sessions lacked applicability to their own learning. Due to changes at the postgraduate level, our program developed an independent PGY-1 medical psychiatry curriculum to address resident needs and improve the learning gap between psychiatry and medicine.

Summary of the Innovation: A scoping assessment was completed by collating focus group feedback and rotation assessments from the previous curriculum. This data grounded the planning and development of a case-based PGY-1 psychiatry curriculum with a medical focus. This innovation relies on a group of interdisciplinary educators from Psychiatry, Emergency Medicine, Neurology and Internal Medicine who actively participated in curriculum development. 15 case-based sessions, including simulation sessions, were created focusing on core topics including cardiac emergencies, seizures, neurological issues, and chronic health issues, situated within psychiatric settings. Significant depth of medical information is provided including labs and images, enhancing learning across both medical and psychiatric objectives. Triple-jump style cases allow for learning and application of knowledge within sessions and aim to improve knowledge retention and application. Each session concludes with formal learning reflections, designed to consolidate knowledge and promote lifelong learning.

Conclusion: This session will discuss the crossdisciplinary collaborative process involved in curriculum development, provide examples of cases developed, and discuss how attendees can use this method to develop robust, cross-disciplinary curricula relevant to learners within their own settings.

\section{DP 7-2 Examining Diagnostic Radiology} Residency Case Volumes from a Canadian Perspective: A Marker of Resident

\section{Knowledge}

Nicholas Cofie Queen's University, Benjamin Kwan Queen's University, Benedetto Mussari Queen's University, Pam Moore Queen's University, Lynne Meilleur Queen's University, Omar Islam Queen's University, Alexandre Menard Queen's University, Don Soboleski Queen's University, Nancy Dalgarno Queen's University

Background/Purpose: New guidelines from the Accreditation Council for Graduate Medical Education (ACGME) have proposed minimum case volumes to be obtained during residency but there are currently no minimum case volumes standards for radiology residency training in Canada. Using data from a pilot study, we examine radiology resident case volumes among recently graduated cohorts of residents and determines if there is a link between case volumes and measures of resident success.

Methods: Resident case volumes for three cohorts of graduated residents (2016-2018) were extracted from the institutional database. Achievement of minimum case volumes based on the ACGME guidelines was performed for each resident. Pearson correlation analysis ( $n=9)$ was performed to examine the relationships between resident case volumes and markers of resident success including residents' relative knowledge ranking and their American College of Radiology (ACR) in-training exam scores.

Results: A statistically significant, positive and strong correlation was observed between residents' case volume and their relative knowledge ranking $(r=$ $0.682, p<0.05)$. Residents' relative knowledge ranking was also strongly and positively correlated with their ACR in-training percentile score $(r=0.715$, $p<0.05)$.

Conclusion: This study suggests that residents who interpret more cases are more likely to demonstrate higher knowledge. This highlights the utility of case volumes as a prognostic marker of resident success. 
The results also underscore the potential use of ACGME minimum case volumes as a prognostic marker. These findings can inform future curriculum planning and development in radiology residency training programs.

\section{DP 7-3 Strengths and challenges of the} learning environment across postgraduate medical education programs

Loni Desanghere University of Saskatchewan, Anurag Saxena University of Saskatchewan, Scott J. Adams University of Saskatchewan

Background/Purpose: The learning environment is recognized as a key aspect of effective postgraduate medical education programs. Less is known regarding how resident perceptions of the learning environment vary across program disciplines. This study sought to explore current strengths and challenges of the learning environment at a Canadian medical school, and compare and contrast how they vary across programs.

Methods: A survey was distributed to all residents at our institution for two consecutive years, with openended questions to elicit strengths and challenges of the learning environment. Survey data was coded using NVivo, and analysis proceeded using a grounded theory approach.

Results: 504 residents participated (58\% response rate). Three themes emerged from analysis, each manifesting as a strength and challenge of the learning environment: interpersonal relationships, program structure and organization, and learning opportunities. Across all programs, residents described approachable attending physicians as a strength of the learning environment. Call structure, including frequency and lack of post-call days, particularly among surgical residents, negatively impacted work-life balance; however, similar challenges were expressed by residents in other disciplines in the absence of frequent call. Internal medicine and surgical residents felt that clinical service demands and "scut work" hindered learning opportunities to a greater extent than family medicine residents.

Conclusion: Dichotomies in how the three themes of interpersonal relationships, program structure and organization, and learning opportunities manifest as a strength or challenge of the learning environment demonstrate many similarities and subtle differences among programs, providing opportunities for institution-wide and program-targeted interventions to improve the learning environment.

\section{DP 7-4 A theoretical model to determine} how CaRMS variables impact medical student matching rates.

Andrew Volk University of Alberta, Hollis Lai University of Alberta, Tracey Hillier University of Alberta

Background/Purpose: Medical students must match and complete a residency before becoming licensed physicians in Canada. Matching is facilitated by rankorder lists submitted concurrently by students and residency programs. Lessons from the Monty Hall problem suggest a possible improvement to the match. We proposed the Monty Hall adjustment to the existing match, with one key difference: student's rank order lists are edited by removing programs that did not rank them prior to running the CaRMS algorithm.

Methods: A Monte Carlo simulation was created to investigate the difference in match rate between the two models. Rank order lists (ROL) for students and programs were generated from distributions emulating known populations. The matching Markets package from $\mathrm{R}$ facilitated the match. Five factors were varied with 100 replications to systematically identify the best scenarios between the two models: standard deviation of student file review scores, number of residency positions per student, number of students interviewed per position, percentage of interviewed students that were ranked, and number of direct entry programs.

Results: Initial results suggest an average of $1.7 \%$ match rate gain using the Monty Hall adjustment across scenarios. Moreover, increasing the number of interviews per student to a ratio of 1.2 or decreasing the number of CaRMS entry programs yielded significant gains.

Conclusion: From the statistical simulation, we recommend further investigation of the Monty Hall model with pre-collected CaRMS data, increasing the 
number of interviews per residency spot, increasing the ratio of residency positions to students to 1.2 , and combining suitable programs into fewer common entry programs.

\section{DP 7-5 Assessment of human trafficking knowledge in medical residents prior to and following completion of an online education module}

Keon Ma University of Alberta, Jahaan Ali University of Alberta, Julianna Deutscher University of Alberta, Jason Silverman University of Alberta, Chris Novak University of Alberta, Sandy Dong University of Alberta, John Chmelicek University of Alberta, Erica Dance University of Alberta, Helly Goez University of Alberta

Background/Purpose: Human trafficking (HT) is prevalent in Canada and victims are predisposed to numerous health concerns. Many encounter healthcare practitioners during captivity, but the level of awareness and knowledge among front line physicians is low. Limited data exists on attempts to address this within residency training programs. Formal curriculum time in residency is limited, so online modules may be a useful alternate modality.

Methods: Family medicine, emergency medicine and general pediatrics residents at the University of Alberta were invited to participate. They completed short surveys including knowledge assessment questions before and after completing an online learning module on HT. Baseline and postintervention changes in both self-reported and tested knowledge were assessed.

Results: 32 residents completed the pre-survey: $6 \%$ self-identified as somewhat knowledgeable on HT and $16 \%$ knew red flags to identify victims. $81 \%$ wanted this topic incorporated into residency training, but only $6 \%$ had previous education in residency, and $25 \%$ in medical school. $13 \%$ were comfortable reporting victims, and $6 \%$ reported knowing how to report. 20 residents completed the post-survey, with improvements in both self-reported $(p<0.001)$.

Conclusion: Baseline HT knowledge in residents providing first contact care appears limited. An online learning intervention significantly enhanced knowledge and confidence in management. Residency programs should consider more HT education to improve competency in care, and an online module was shown to be a feasible approach.

DP 7-6 Witnessed and Experienced forms of Racism in Post-graduate Medical Education at a Canadian Academic Centre

Lara Cooke University of Calgary, Janet de Groot University of Calgary, Lynden Crowshoe University of Calgary, Parichita Choudhury University of Calgary

Background/Purpose: Systemic racism has been noted to be pervasive in our society and found to be a social determinant of health for patients. Racism experienced by healthcare staff impacts wellbeing of patients and healthcare providers. The objective of this study was to estimate the prevalence of witnessed or experienced racial discrimination among post-graduate medical trainees and their response to these encounters at one large academic health centre.

Methods: Post-graduate Medical trainees $(n=840)$ at the University of Calgary were surveyed using a tenquestion questionnaire. The survey was distributed by the Post-graduate Medical Education office. Participation in the study was entirely voluntary and all data was collected anonymously. Data was collected through the online survey Platform REDCap. This study was approved by the research ethics board at the University of Calgary.

Results: The response rate for this survey was $12 \%$. $52 \% \quad(n=52)$ of responders indicated they had witnessed "everyday" or overt racism during their post-graduate medical training. Witnessed microaggressions $(n=34)$ were most commonly reported by trainees followed by experienced microaggression $(n=17)$, witnessed overt racism $(n=16)$, and experienced overt racism $(n=7)$. Most respondents ( $n=38$ witnessed and $n=15$ experienced) did not take action in response.

Conclusion: Our study shows that systemic racism is common and prevalent in post-graduate medical training in Canadian healthcare. This is directed towards patients, trainees and other members of the healthcare team and constitutes "everyday racism" 
most frequently, consistent with studies in the United States. Trainees frequently did not take action in these situations given the hierarchical nature of medicine and fear of repercussions.

\section{DP 7-7 Strategies to Increase Patient Safety} Event Reporting By Resident Physicians: A Narrative Review

Ulemu Luhanga Emory University School of Medicine, Maria Aaron Emory University School of Medicine, Adam Webb Emory University School of Medicine

Background/Purpose: Resident physicians are often on the front lines of patient care and are likely to witness adverse events first hand. The goal of this narrative review was to examine the current literature with respect to strategies to increase patient safety event reporting by residents.

Methods: We used CINAHL, EMBASE, PsycINFO, and PubMED. The search was limited to English-language articles published in peer-reviewed journals through April 2018. Participants included "residents, trainees, fellows" and key terms used to identify interventions were: "event reporting, patient safety reporting, incident reporting, adverse event, medical error". To guide study appraisal and synthesis, we adapted Peter's Five Strategies for Encouraging Self-Protective Behavior framework.

Results: We identified 59 articles that described strategies used to increase event reporting. The most common strategy, surveys and interviews, were instrumental in assessing current barriers to reporting and/or the trainees' understanding of patient safety and for increasing awareness of event reporting. Behavior modeling and training was also essential for educational purposes, addressing deficits in knowledge and/or specific hospital systems used for event reporting. Lastly, frequent messaging was needed to reinforce behaviors that are often not part of routine patient care. One limitation of our review was that we did not find interventions which occurred after the submission of a safety event.

Conclusion: The ideal, most successful sustainable interventions will be those that combine strategies that minimize time for busy physicians, incorporate constant reminders in already existing medical records, and become part of normal workflow in patient care.

DP 7-8 Variables qui influencent la rétroaction aux résidents: une recension systématique des écrits

Manon Denis-LeBlanc University of Ottawa, Lyne Pitre University of Ottawa, Maud Mediell University of Ottawa, Adjo David University of Ottawa, Salomon Fotsing University of Ottawa, Eric Dionne University of Ottawa

Background/Purpose: Les précepteurs jouent un rôle déterminant dans l'apprentissage et développement des compétences essentielles pour être un médecin qualifié. Dans ce contexte, la rétroaction formative constitue un élément clé dans le développement des compétences cliniques chez un futur médecin. On se demande donc ce qu'est une rétroaction efficace. Ce projet visait à identifier les principales variables susceptibles d'influencer la rétroaction.

Methods: Nous avons effectué une recension systématique des écrits en anglais et en français à travers des bases de données scientifiques suivantes : ERIC, CINHAL, PsyInfo, PubMed, Ovid, ainsi que dans la littérature grise. L'ensemble des articles sélectionnés a permis de classer les informations en dix (10) catégories.

Results: Sur les 340 articles identifiés, seuls 104 articles ont été retenus pour l'analyse. Les variables qui semblent le plus affecter la rétroaction sont: le milieu de formation, les objectifs de la rétroaction, le type de rétroaction, le niveau de formation des précepteurs, la fréquence de la rétroaction, le contexte de pratique, la nature des outils, l'utilisation de la technologie, l'étudiant, les outils utilisés.

Conclusion: Nos analyses révèlent 10 variables qui sont les plus importantes dans l'efficacité de la rétroaction. 


\section{DP 8-1 The Impact of Practising Empathy on a Physician's Individual Personhood}

Laura Tan Yong Loo Lin School of Medicine, National University of Singapore, Laura Tan Yong Loo Lin School of Medicine, National University of Singapore, Lorraine Tan Yong Loo Lin School of Medicine, National University of Singapore, Yihan Khoo Yong Loo Lin School of Medicine, National University of Singapore, Ying Pin Toh National University Hospital, Singapore, Annelissa Chin Yong Loo Lin School of Medicine, National University of Singapore, Lalit Krishna National Cancer Center Singapore; Yong Loo Lin School of Medicine, National University of Singapore; Center for Biomedical Ethics, National University of Singapore; Duke-NUS Graduate Medical School

Background/Purpose: Practicing empathy is an imperative in the practice of humanistic medicine. However, the central role of empathy has been thrown into question in part due its associations with physician burnout and compassion fatigue. This scoping review aims to provide a clear framework to consolidate and understand the effects of empathy on a physician's individual personhood, as understood through Krishna et al.'s Ring Theory of Personhood.

Methods: A search of 7 databases (Pubmed, Cochrane, ERIC, Embase, PsycINFO, CINHAL and Google Scholar) was conducted till 17 November 2018. Papers were screened using a set of inclusion and exclusion criteria, and corresponding data was extracted and analyzed.

Results: 7663 titles and abstracts were retrieved, of which 108 articles were analysed. Our research revealed that when a physician practices empathy, it impacts 3 broad aspects of his individual personhood. Firstly, it changes his personality, values, ethics and morals. Secondly, it impacts his clinical competency (in terms of clinical acumen and time management) and reputation. Lastly, it affects his physical and emotional well-being, with implications for burnout, compassion fatigue and compassion satisfaction. These results demonstrate that practicing empathy has significant impact on a physician's individual personhood, providing evidence that empathy should be encouraged and nurtured in medical education as it facilitates the acquisition of values imperative to a physician's personal growth and clinical practice.

Conclusion: The practice of empathy should be regarded as a key competency to be attained in medical education as it has significant effects on a physician's individual personhood and eventual medical practice.

\section{DP 8-2 The Impact of Practising Empathy on} a Physician's Relational and Societal Personhood

Laura Tan Yong Loo Lin School of Medicine, National University of Singapore, Lorraine Tan Yong Loo Lin School of Medicine, National University of Singapore, Yihan Khoo Yong Loo Lin School of Medicine, National University of Singapore, Ying Pin Toh National University Hospital, Singapore, Annelissa Chin Yong Loo Lin School of Medicine, National University of Singapore, Lalit Krishna National Cancer Centre Singapore; Yong Loo Lin School of Medicine, National University of Singapore; Centre for Biomedical Ethics, National University of Singapore; Duke-NUS Graduate Medical School

Background/Purpose: While empathy is clearly essential to the humanistic practice of medicine, it is less intuitive how empathy influences a physician's relational and societal personhood. Our scoping review explores how practicing empathy interacts with and affects elements in a physician's relational and societal world by utilising concepts of Krishna et al.'s Ring Theory of Personhood.

Methods: 7 databases (Pubmed, Cochrane, ERIC, Embase, PsycINFO, CINHAL, Google Scholar) were searched till 17 Nov 2018. Papers were screened using the determined inclusion and exclusion criteria. The corresponding data was extracted and analyzed using a coding framework.

Results: 7663 titles and abstracts were retrieved, of which 108 articles were analysed. Thematic analysis revealed that practicing empathy had implications on 6 aspects of a physician's relational and societal world: 1) relationship with family, 2) relationship with colleagues, 3) teaching role, 4) physician-patient relationship, 5) professional obligations and 6) guardian of societal resources. Our results demonstrate that practicing empathy has significant 
impact on a physician's interactions with the world around him, providing evidence for the notion that empathy should promoted in medical education as it is a key driver and foundation of humanistic care and effective relationships.

Conclusion: Practicing empathy impacts a physician's relational and societal personhood. Medical education should prioritise nurturing empathy as a key competency, as it has significant effects on a physician's relational and societal personhood and can drive humanistic goals of healthcare.

DP 8-3 Récits de soi récits de soins: Nouvelles perspectives pédagogique et éthique

Annie Descôteaux Université de Montréal, Alexandre Berkesse Centre d'excellence sur le partenariat avec les patients et le public, Marie-Pierre Codsi Université de Montréal, Antoine Payot Université de Montréal, Philippe Karazivan Université de Montréal, Vincent Dumez Université de Montréal, Amélie Du PontThibodeau Université de Montréal, Nathalie OrrGaucher Université de Montréal, Melissa Taguemout Université de Montréal, Anne-Sophie Eymard Université de Montréal, Mathieu Jackson Université de Montréal, Clara Dallaire Université de Montréal, Louise Nicaise Université de Montréal

Background/Purpose: L'enseignement de la communication et des enjeux éthiques dispensé aux étudiants reste un défi en éducation médicale. Depuis 2014, les étudiants de I'Université de Montréal produisent, dans le cadre d'atelier d'éthique clinique, des vignettes portant sur des situations humaines complexes vécues pendant leur externat. Plus de 1500 vignettes écrites par les étudiants, ainsi qu'une trentaine de vignettes écrites par les patients partenaires coformateurs ont été le tremplin vers une initiative pédagogique, sur une base pleinement volontaire.

Summary of the Innovation: Certaines vignettes marquantes ont retenu l'attention des coanimateurs et suscité intérêt et discussions. Les membres de la Direction collaboration et partenariat patient (DCPP), le Bureau de l'éthique clinique (BEC) de la faculté de médecine de I'Université de Montréal ainsi que des comédiens se sont réunis pour coconstruire avec les étudiants et les patients une soirée théatre de mise en lecture de ces vignettes. L'importance des thèmes, l'impact émotif du contenu et la puissance pédagogique portés par ces vignettes ont mené l'équipe à vouloir mettre en lumière les éléments éthiques. Cette soirée a été présentée dans le cadre d'un colloque en pédagogie médicale (SIFEM) en mai 2019 à Montréal.

Conclusions: Fermement ancrée dans l'approche pédagogique narrative, cette activité de mise en scène des vignettes offre de nouvelles perspectives pédagogiques et éthiques pour les étudiants, les enseignants et le public. Cette activité pédagogique sera reprise à l'Université de Montréal pour l'année 2020.

DP 8-4 "Learning to Play Again": Medical Student Clowning Elective and Lollipop Moments Day with Molly Penny the Therapeutic Clown at the Children's Hospital of Eastern Ontario (CHEO)

Yipeng Ge University of Ottawa, Ruth Cull Children's Hospital of Eastern Ontario (CHEO)

Background/Purpose: As a medical student, I watched in awe and curiosity in the interactions that Molly Penny, the therapeutic clown, fostered with those around her. Therapeutic clowning has been shown to be effective in providing psychosocial support to children, youth, and their families. Learning from Molly Penny as a clown-in-training resulted in one of the most impactful, informative, and transformative experiences for me as a medical trainee and as a person. The "lollipop moment" is a concept that small acts of kindness, humanity, and leadership can make profound positive impacts on others, shared by Drew Dudley, a speaker and author.

Summary of the Innovation: The 10-hour therapeutic clowning elective for pre-clerkship medical students aims to help develop listening, sensitivity, creativity, and communication skills to effectively build rapport. As well as, encourage reflecting on the role of laughter, play, and humour in healing and reconciling aspects of psychosocial and physical determinants of health. A Lollipop Moments Day, the sharing of stories and laughter through lollipops, was brought to 
fruition with the help of hospital staff and children, youth, and their families.

Conclusion: We can help facilitate the sharing of stories that shed more light on the experiences that make us human and connected through something as simple as sharing a lollipop or a sticker, to enhance and make more bearable the moments in hospital. We hope to help catalyze a movement and acknowledgement that small gestures, words, and actions can have a powerful cumulative effect on society and our culture of how we interact and treat one another, starting in the hospital.

\section{DP 8-5 Developing medical students' leadership and collaboration competence through project management in the community}

Guylaine Séguin Université de Sherbrooke, Paul Bessette Université de Sherbrooke, Eve-Reine Gagné Université de Sherbrooke, Sylvie Houde Université de Sherbrooke, Sylvie Mathieu Université de Sherbrooke, Sébastien Roulier Université de Sherbrooke, Geneviève Petit Université de Sherbrooke, Linda Pinsonneault Université de Sherbrooke, Ghislaine Houde Université de Sherbrooke

Background/Purpose: Dans le cadre de la refonte du programme de médecine fondée sur un parcours de professionnalisation, nous avons construit une activité pédagogique dédiée à l'acquisition des compétences en gestion de projet et à la mise en œuvre de projets dans la communauté.

Summary of the Innovation: L'activité se déroule sur une année de formation et comprend 4 phases au projet : l'identification, la planification, la réalisation et la clôture du projet. Chaque projet est réalisé par une équipe de 5 à 8 étudiants supervisés par un professeur et en partenariat avec un organisme communautaire. Les méthodes pédagogiques incluent des ateliers pratiques et réflexifs, des rencontres avec les superviseurs et les partenaires. Les idées de projets sont sélectionnées en fonction des objectifs, des besoins de la communauté, de l'impact et de la faisabilité. La planification comprend l'échéancier, l'analyse des parties prenantes, les prévisions budgétaires, l'analyse des risques et les indicateurs de performance. La réalisation comprend le plan de communication, la gestion des problèmes et des risques ainsi que le suivi de l'échéancier, du budget et de la qualité des livrables. Le projet se termine par la production d'un rapport de clôture et des présentations en classe et auprès des partenaires. L'évaluation multi sources s'appuie sur la rétroaction par les pairs et les partenaires, les observations directes des superviseurs et les travaux livrés à chaque phase du projet.

Conclusion: La participation aux différents ateliers de même que la mise en oeuvre des projets permettent le développement de meilleures pratiques de gestion, $\mathrm{du}$ leadership, du travail collaboratif et de l'engagement communautaire. L'activité permet aussi de créer des liens entre la clientèle étudiante, la communauté et la Faculté.

DP 8-6 Developing the roles of manager and leader for undergraduate medical students in a competency-based education program

Guylaine Séguin Université de Sherbrooke, Paul Bessette Université de Sherbrooke, Gabrielle Trépanier Université de Sherbrooke, Eve-Reine Gagné Université de Sherbrooke, Sylvie Mathieu Université de Sherbrooke, Sylvie Houde Université de Sherbrooke, Damien Bélisle Université de Sherbrooke, Ghislaine Houde Université de Sherbrooke

Background/Purpose: Le rôle de gestionnaire fait partie intégrante d'une pratique médicale durable. II gagne à être explicité et développé durant tout le cursus de formation et réfère aux rôles CanMeds de leader, de collaborateur et de professionnel. Dans le cadre de la refonte du programme médecine, nous avons construit des activités pédagogiques intégrées et longitudinales soutenant le développement du rôle de gestionnaire.

Summary of the Innovation: Les activités pédagogiques préparent les étudiants à gérer leur pratique médicale et à exercer leur leadership au sein du système de santé. Elles s'appuient sur les fonctions de gestion : planifier, développer (mise en œuvre), contrôler et autoréguler et supportent la pratique réflexive et le développement de l'agir compétent en situation réelle. La lère étape adresse la gestion 
individuelle des études et de la vie personnelle incluant les styles de leadership, les meilleures pratiques de gestion du temps, des finances, du stress et des stratégies d'études. La 2e étape est consacrée à la gestion d'un projet que les étudiants réalisent en équipe dans la communauté. La 3e étape aborde les règles, le fonctionnement et l'organisation des milieux de soins afin d'amorcer la gestion clinique et l'amélioration de la qualité des soins. L'évaluation est fondée sur des examens, des travaux écrits, des livrables, des analyses réflexives ainsi que sur des évaluations multi-sources.

Conclusion: Le développement du rôle de gestionnaire et de leader s'est réalisé dans des activités adaptées à chaque niveau de formation. Les professeurs soulignent leur pertinence pour la pratique et le mieux-être des futurs médecins. Les étudiants apprécient la mise en application des meilleures pratiques de gestion de même que les occasions de développer leur leadership et le travail en équipe.

\section{DP 8-7 Advocacy in community-based service learning: perspectives of} community organizations

Judy Truong University of Toronto, Vanessa Sheng University of Toronto, Priya Sandhu University of Toronto, Yasamin Sadeghi University of Toronto

Background/Purpose: Community-based service learning (CBSL) placements could be designed to train medical students as "Health Advocates". Although the primary intended beneficiary of service-learning is both the recipient of the services and the provider, little is known about the experience of community partners. Purpose is to understand how partner community organizations (PCOs) perceive their role in service-learning, the benefits of participating in service learning with medical students, barriers and facilitators to this partnership, and whether CBSL is effectively teaching medical students about their role in advocacy.

Methods: This is a qualitative program evaluation of a service-learning course in the University of Toronto medical school. In-depth interviews were performed with 5-10 community partners identified through purposive sampling strategies. Data were recorded, transcribed and coded using deductive and inductive coding by two independent coders. Codes will be analyzed for themes, supported by group discussion and memos.

Results: Themes included advocacy, service learning and course improvement feedback. A few PCOs described CBSL as a mutually beneficial activity but felt their goal was to help students be "better" doctors. Many PCOs described themselves as "medically-adjacent" and tended to give students medically-related tasks. The PCOs that participated as longitudinal co-tutors for delivery the $\mathrm{HC}$ curriculum felt empowered and several requested more information on the medical students' in-class curriculum to improve their role in educating students on advocacy.

Conclusion: In identifying the experiences of community partners, service learning curricula can be refined to address the needs of community agencies and improve advocacy training for medical students.

\section{DP 9-1 Understanding resident perspectives} of critical events during training

Kevin Venus University of Toronto, Nadine Abdullah University of Toronto, Lindsay Melvin University of Toronto

Background/Purpose: As residents progress through their training, they experience certain events that have a lasting impact. These 'critical events' may either be associated with positive or negative emotions. These events stand out due to their uniqueness, emotional context, clinical situation or educational weight and are consolidated after a period of active reflection. This project seeks to understand 1) the types of critical events residents experience during their training, 2) if these events are recognized by their supervisors and if so, how this modulates residents' reflection on them, and 3 ) how these events relate to residents' development of professional identity.

Methods: Participants were recruited via email advertisements and purposeful sampling. Data was collected via guided interviews of senior internal medicine residents. Participants described a 'critical event' in their training and asked a series of standardized and follow-up questions by a near-peer 
researcher. Twelve interviews were analyzed utilizing constructivist grounded theory after being conducted to thematic saturation.

Results: Analysis of the narratives showed that $7 / 12$ (58\%) of the critical events were positive experiences. Positive events are characterized by responsibility, transition points and are grounded in the patient relationship. Negative events are characterized by isolation and perceived lack of supervisor support. All events are not explicitly recognized by supervisors.

Conclusion: Senior internal medicine residents easily identified critical events from training. Positive and negative events are experienced and reflected upon differently. Residents desire supervisors to play an active role in fostering reflective practice, which may mitigate future negative effects of these experiences. These experiences may be better understood utilizing transformative learning theory.

DP 9-2 Building from the Ground Up: Designing Educational Material Based on Perceived and Unperceived Needs in Malignant Hematology Training in

\section{Canadian Hematology Residents}

Wilson Lam University of Toronto, Hassan Sibai University of Toronto

Background/Purpose: Malignant Hematology is in a new era of exciting novel treatment regimens and modalities, including CAR-T (Chimeric Antigen Receptor T-cells) and BiTE (Bi-specific T-cell Engaging) antibodies. Current trainees require an everincreasing knowledge and skillset to deliver high quality care to more complex patients. There is limited evidence on the educational needs of hematology residents with these emerging complexities. Moreover, these educational needs themselves are poorly-defined. As a first step, we sought to perform a detailed needs assessment to identify knowledge gaps in our learners. It is our intention to use this information to aid in developing an educational tool incorporating these novel elements.

Methods: Every year Hematology residents in Canada attend the National Hematology Retreat in Toronto, Ontario for a weekend of educational activities, which also serves as preparation for the Royal College of Physicians and Surgeons Hematology certification exam. Residents were invited to participate in a questionnaire to identify perceived and unperceived needs. They were asked to select topics of perceived needs from a pre-selected list. This was followed by a knowledge assessment using case-based questions in leukemia, myeloma, Iymphoma, and Blood and Marrow Transplantation [BMT]. The study is approved by the University of Toronto Research Ethics Board. Data were analyzed descriptively as needed. Mean total scores from the case-based questions were compared between training years using one way ANOVA. All statistical calculations were performed using SPSS version 26.

Results: Over two years we surveyed from 85 out of approximately 150 Canadian Hematology residents attending the retreat. Among the respondents, 2 were PGY-3 or lower, 24 were PGY-4, 31 were PGY-5, and 28 were PGY- 6 and above. 3 respondents had done the survey previously. Of our pre-selected topics list, residents perceived the most common knowledge gaps existed in management of BMT complications, followed by molecular testing (especially genomics), and novel immune and cellular therapies. The top choices differed in the PGY-4 year (BMT complications, novel immune and cellular therapies and emergency AML complications, Figure 1).

Conclusion: Needs assessments are a useful tool in assessing background knowledge, identifying perceived and unperceived needs of trainees, and can be used towards creating a resource that accounts for learning priorities. Our needs assessment of hematology residents across Canada demonstrated that: 1) Knowledge gaps exist among residents at different levels of training, particularly in BMT, compared to other areas of Malignant Hematology. Moreover, this was perceived by residents themselves. 2) Learning priorities of residents may change over the course of their training. Our next step is to assess what specific gaps residents have in molecular testing and CAR-T cells and potentially create an electronic based learning tool. 
DP 9-3 Resident opinions and educational experience of a Mixed Night-Float System for general surgery resident call

Robin Ralph-Edwards Western University, Michael Ott Western University, Julie Ann VanKoughnett Western University

Background/Purpose: There is ongoing debate regarding the optimal approach to overnight call, given the increased interest in duty hour restrictions among surgical programs in Canada. Thus far General Surgery programs have avoided a night-float approach. The purpose of this study is to evaluate a mixed short-segment night-float (MSSNF) call schedule compared to traditional call in subjective educational experience among junior residents.

Methods: PGY1 residents on general surgery were surveyed at completion of block on their satisfaction with educational experience, assessment of workload and ability to attend teaching after either a block of traditional 24-hour call schedule or the MSSNF schedule (5 consecutive $5 \mathrm{pm}-7 \mathrm{am}$ in-house shifts with daytime alloted to post-call recovery, and two non-consecutive 24-hour weekend shifts per month).

Results: Of the 44 surveyed, $41 \%$ were surgical residents. Residents ranked overnight consults as the greatest source of learning on GS. More residents in MSSNF felt overnight workload was less compared to other rotations ( $41 \%$ vs. $5 \%$ ), and $36 \%$ more felt call was less with MSSNF. More found overnight work a useful educational experience with MSSNF (91\% vs. $41 \%$ ), and $91 \%$ were able to attend teaching (vs. $86 \%$ traditional).

Conclusion: MSSNF residents felt a subjective decreased burden of overnight workload, increased subjective educational experience and felt they were doing less call overall compared to traditional schedules.

Summary: MSSNF is a feasible and well-received method of resident call scheduling which may improve subjective opinions of the generally strenuous general surgery call.
DP 9-4 Observation Directe à l'urgence : un milieu favorable?

William Bédard Michel Université de Montréal, Véronique Castonguay Université de Montréal, Pierre Desaulniers Université de Montréal, Éric Piette Université de Montréal, Massimiliano Iseppon Université de Montréal

Background/Purpose: L'observation directe (OD) est un élément clé dans les stratégies d'enseignement et d'évaluation utilisées en approche par compétences. Les bénéfices de I'OD ont déjà été rapportés dans la littérature. Toutefois, à l'urgence, les résidents sont seulement observés $3,6 \%$ du temps. Une des principales barrières étant l'achalandage et la crainte d'une diminution du débit. Par contre, cela n'a jamais été formellement étudié dans le passé. L'objectif primaire de l'étude est d'évaluer l'impact de I'OD sur le débit à l'urgence. L'objectif secondaire est l'évaluation de l'impact de I'OD sur l'exposition des résidents.

Methods: Il s'agit d'un projet de cohorte prospective unicentrique impliquant des résidents $\mathrm{R} 2-\mathrm{R} 4$ en médecine d'urgence spécialisée. Ceux-ci ont accompli deux types de quarts de travails. Ceux usuels (U), sans modification par rapport à la pratique établie, et ceux d'OD où le quart entier était effectué en OD. Pour l'analyse statistique, des tests de Mann-Whitney ont été utilisés.

Results: $18 \mathrm{U}$ et 26 OD furent effectués. Une différence significative fut démontrée en défaveur de I'OD en regard du débit total $(U=15 ; O D=12, p=$ $0,02)$. Par contre, l'exposition des résidents est significativement plus élevée en faveur de I'OD ( $U$ = 7 ; OD $=9 p=<0,001)$.

Conclusion: Notre étude confirme l'hypothèse que I'OD est associée à une diminution du débit tout en augmentant significativement l'exposition des résidents. Cela s'ajoute aux avantages de I'OD déjà reconnus. Une réflexion devra être effectuée dans les milieux universitaires pour déterminer comment maximiser I'OD sans nuire au flot global de l'urgence. 
DP 9-5 Transitioning towards best practices in residency application and selection, a work in progress: Snapshot of a Canadian university's residency programs admission processes

Patrice Chrétien Raymer Université de Montréal, Jean-Michel Leduc Université de Montréal

Background/Purpose: Best practices in applications \& selection (BPAS) for postgraduate medical education (PGME) are now promoted by the AFMC and CaRMS. In this study, we aimed to identify areas for improvement (AFI) in the PGME selection processes at Université de Montréal (UdeM).

Methods: An online questionnaire was sent to 73 PD at UdeM to assess selection procedures related to BPAS.

Results: 54 PD (74\% participation) completed the online survey. Most programs had well-defined selection tools, file review steps and desired candidate characteristics (94\%, $80 \%$ and $61 \%$, respectively), but fewer programs had clear "do not rank" (DNR) criteria, procedures to disclose conflict of interests (COI) or methods to manage data obtained outside the formal selection process (32\%, $11 \%$ and $2 \%$, respectively). Only $55 \%$ of programs openly disclosed to candidates how they generated the final ranking list. Less than $2 / 3$ of programs formally trained their admission committee members for file review and interview processes $(57 \%$ and $41 \%$, respectively). Only $8 \%$ of interviews were performed completely independently, without access to the rest of the applicant's file. A minority of programs (10\%) performed psychometric analysis of their selection tools' data.

Conclusion: This study identified many AFI and challenges in our PGME selection processes that will help to guide BPAS implementation in our institution, including improving transparency for some elements (DNR criteria, COI disclosure and final ranking method), increasing admission committee training and obtaining more resources for psychometric analysis of their selection tools.

\section{DP 9-6 Pots Maison - une initiative étudiante}

Ariane Bergeron Université de Montréal, Judith Lefebvre Université de Montréal, Marie-Ève Villeneuve Université de Montréal

Background/Purpose: Le nouveau guide alimentaire canadien publié en janvier 2019 apporte un changement de paradigme en santé nécessaire. Dû à un rythme de vie effréné, l'intégration de saines habitudes alimentaires est plus difficile à concilier au quotidien. Le désir d'efficacité de la population canadienne influence leur prise de décision, de l'épicerie à l'assiette, en priorisant les produits transformés pouvant être préparés et consommés rapidement. À l'aide de 3 sphères d'intervention, soit l'érudition, le partage des connaissances et l'implication sociale, Pots Maison répond à une lacune entourant les connaissances en nutrition de la communauté médicale et du grand public dans le but de démocratiser l'accès à l'information entourant la saine alimentation.

Summary of the Innovation: L'élément rassembleur des 3 sphères d'intervention est le e-book, contenant 10 recettes et 7 capsules de professionnels de la santé. Grâce à la promotion du livre à l'école, sur les médias sociaux et à la radio locale, plus de $3000 \$$ de dons ont été remis au centre de pédiatrie sociale de Trois-Rivières. Pour inciter les étudiants et les jeunes de la pédiatrie sociale à cuisiner avec plaisir, 2 ateliers de cuisine ont été organisés. Pour démystifier le guide alimentaire, une nutritionniste a donné une conférence à ce sujet. En 2019, il y a eu 50 participants aux activités et plus d'une centaine de personnes qui ont accédé au livre.

Conclusion: Par une réponse interdisciplinaire adaptée, ce projet propose une solution à la complexité de l'information sur la nutrition. Les participants montrent un sincère intérêt à la saine alimentation et la préparation collective de repas. L'encadrement offert permet de développer une meilleure confiance en cuisine. Le projet sera renouvelé pour l'année à venir avec l'ajout d'enjeux alimentaires d'actualité. 
DP 9-7 Learning to interpret the information gathered through palpation: a meta-analysis

Phillip Surmanowicz University of Alberta, Liam Rourke University of Alberta, Ron Damaant University of Alberta

Background/Purpose: Educators have effective methods for imparting the knowledge and procedures necessary for effective palpation. They have not settled on methods for cultivating tactile expertise - the ability to appreciate subtle distinctions among the perceptual features that distinguish normal from abnormal tissue. We conducted a metaanalysis to determine the scope and effectiveness of educational activities used to develop tactile expertise.

Methods: We searched MEDLINE comprehensively using terms cognate with education and palpation and selected those that compared participants' abilities to interpret the diagnostic information gathered through palpation before and after training. We excluded studies that measured only changes in knowledge, skills, or attitudes. We extracted information about the educational objective, the learning activities, and their effect.

Results: Twenty-three studies involving 1,466 participants met our inclusion criteria. They focused predominantly on three educational objectives: the ability to detect masses in breast tissue, evaluate prostate tissue, and locate anatomical structures. Three educational activities, implemented in tandem, were used to impart these abilities: lectures, demonstrations, and guided practice. The effect of these activities, pooled across studies that compared the abilities of trained to untrained participants was large (SMD $=0.93([95 \% \mathrm{Cl}, 0.80,1.05])$.

Conclusion: Brief interventions of have a large effect on the abilities of novices to interpret the information they gather with their fingers and hands, particularly when the abilities are measured immediately after training. The abilities are inadequate for clinical decision-making, but they could be improved by recognizing the importance of tactile expertise in palpation and systematically designing instruction for this outcome.
DP 9-8 The Role Model Moms PostSecondary Academy: An MD ProgramUniversity-Community Partnership Promoting Higher Education for Mothers Completing Their High School Equivalency

Casey Goldstein University of Limerick, Roxanne Wright University of Toronto, Tianyue Wang McMaster University, Danielle Thibodeau No current affiliation, Joyce Nyhof-Young University of Toronto

Background/Purpose: The University of Toronto's MD Program collaborates with local community agencies, organizations and municipal governments to work on the issues they face to enhance community health and institutional connectivity, while identifying experiential learning opportunities to engage students in the communities within which they learn and live. The Role Model Moms (RMM) program supports low-income mothers completing their General Equivalency Diplomas. RMM and the University of Toronto's MD program collaborated to create a program providing tailored information on post-secondary education (PSE) for RMM participants.

Summary of the Innovation: A collaborative, community-engagement approach was utilized to develop the RMM Post-Secondary Academy event, which sought to bridge gaps in existing PSE orientation resources targeting this marginalized group. The half-day event has run for 3 years involving 42,45 , and 38 women, respectively, and numerous undergraduate and MD student volunteers. Events were evaluated via facilitator debriefing sessions, and participant post-event surveys and interviews, with results analyzed using basic statistics and descriptive thematic analysis. Participants gained a positive understanding and outlook on PSE. Their concerns regarding PSE included family, financial, and academic barriers. Event feedback informed program development.

Conclusion: Collaboration enabled minimal strain on existing PS resources, individual facilitators and student volunteers, while community-engagement ensured content was relevant to the target audience. This pipeline program provides a replicable model for responsive community programming and may serve 
as a framework for future programs to increase access, equality and diversity in PS for underrepresented groups.

\section{DP $\quad 9-9$ Key Conceptualizations of}

\section{Professional Identity Formation: A Scoping}

\section{Review}

Kimberley MacNeil University of British Columbia, Maria Hubinette University of British Columbia, Sandra Jarvis-Selinger University of British Columbia

Background/Purpose: A recent explosion in calls for supporting developing physicians' professional identity formation (PIF) in the medical education literature has been followed by an abundance of educational innovations purporting to foster medical students' PIF. However, between the flurry of interest in supporting PIF and related innovations there exists a gap in our understanding of the ways in which theory building and empiric research are converging. We present the results of a scoping review which examines the landscape of PIF research in medical education so as to orient medical educators and researchers to needed next steps.

Methods: This study followed a step-wise scoping review methodology which first involved the research team identifying the research question. We wanted to understand how the conversation around professional identity formation had evolved in the medical education literature over the past ten years, how the current conversations are framed, and what gaps are apparent in the literature. In keeping with scoping review methodology, a wide approach was developed in order to ensure breadth. The process of delimiting the search parameters was iterative; continued conversations between the researchers determined general scope as well as implications of various stances. Inclusion and exclusion criteria were applied to the complete list of 256 studies resulting in the extraction of 166 number of articles. A total of 90 articles were included and Copies of full articles were read by all researchers as we engaged in a more indepth analysis.

Results: Qualitative data were extracted from the publications, analyzed and sorted according to identified themes. The information collected about empiric research papers were - Study "demographics" (title, author(s), publication year, journal, setting) . Purpose of study - Study population - Intervention or phenomenon under study . Methodology and methods - Outcomes . Relevant results The information collected from non-empirical studies included: . Study "demographics" (author(s), publication year, journal, setting) · Purpose/objective of article - Context - Type of article (commentary, intervention/innovation description etc) . Framework/ontology/epistemology/model Implications

Conclusion: Overall this scoping review found a paucity published papers specifically outlining why it is important to study PIF. Most published studies left this for the reader to make major assumptions. As well conceptual frameworks were lacking in the reviewed papers. There were also little articulation of what 'makes a good physician" in other words, what is the goal of professional identity formation for those training to become physicians in the future. Related to this there is little discussion of how medical education is supporting the development of these traits. Finally, outcome studies of PIF curriculum and interventions are needed.

\section{DP 10-1 Medical Student Internship Experience: Using Knowledge Translation to Disseminate Lessons Learned}

Sara Trincao-Batra University of Ottawa, Kyung Joon Mun University of Toronto, Tracy Pham University of Alberta, Tunde Gondocz Department of Practice Improvement, Canadian Medical Protective Association, Allan McDougall Department of Medical Care Analytics, Canadian Medical Protective Association

Background/Purpose: Finding research opportunities often occurs as a necessary expectation in medical school. Many would consider these placements as part of the hidden curriculum; although not a part of most official undergraduate curricula, they are an expectation for staying competitive when applying to residency programs. However, little guidance exists for medical students, undergraduate educators, or clinical researchers interested in creating valuable non-traditional research experiences. 
Summary of the Innovation: The aim of this project is to use knowledge translation (KT) science to describe the content knowledge and practical skills acquired during the Canadian Medical Protective Association's (CMPA) medical student internship; a unique summer program designed to provide undergraduate medical students with an non-traditional training experience in a non-profit, medico-legal setting. Our project utilizes the KT Planning Template and KT Game from the Sick Kids Learning Institute as a framework to provide a rich description of the CMPA's 2019 summer program as well as the cumulative reflections of three undergraduate students.

Conclusion: This project demonstrates that students gained valuable content knowledge and practical skills aligned with CanMEDS roles during this summer internship. It also provides a helpful tool for medical educators interested in a design framework for future undergraduate medical student opportunities. A preliminary internal evaluation demonstrated that this KT framework was effective in disseminating information and increasing awareness of the knowledge and skills acquired during the medical student experience.

\section{DP 10-2 Decrypting Clerkship: A Novel Resident-Led Program to Facilitate the Transition to Clinical Clerkship}

Leah Kosyakovsky University of Toronto, Michael Ruiz University of Toronto, Jonah Himelfarb University of Toronto, Healey Shulman University of Toronto, Mark Bonta University of Toronto

Background/Purpose: The entrance to clerkship represents a challenging transition between the didactic preclinical curriculum and the unfamiliar practical skill-set of patient care. A pilot resident-led initiative was created to stimulate process learning of these under-represented day-to-day skills, including creating clinical assessments, navigating documentation, and medical communication.

Summary of the Innovation: The pilot initiative was created as a longitudinal opt-in program for secondyear students over the academic year. Four smallgroup resident-led sessions were run, each focused on developing separate practical clinical skills through interactive cases. Given the success of this program, it was subsequently incorporated as a mandatory workshop in the University of Toronto's formal Transition to Clerkship undergraduate curriculum. The goals of the workshop were to orient new clerks to their upcoming clinical responsibilities and develop a rotation-specific approach to a new patient. Using a novel framework for patient assessment (abbreviated EARWORM), students were guided through two interactive cases. Students created their own plans and received feedback on their oral presentations. Students were then surveyed on their confidence with the relevant skills.

Conclusion: After the workshop, student respondents felt more comfortable using an organized approach to a new patient (90.3\%), creating a differential diagnosis (77.4\%), formulating a plan (74.2\%), and presenting a succinct case summary (90.3\%). $91 \%$ stated they found practical resident feedback useful and $84 \%$ felt more comfortable entering clerkship. Overall, our program has strong potential to strengthen the transition to clinical practice, highlighting the value of simulated case practice and personalized near-peer mentorship in enhancing clinical competence.

\section{DP 10-3 Finding Flow: A Systematic Review on Cognitive Flow in Healthcare}

Stephanie Jiang Queen's University, Sydney McQueen University of Toronto, Aidan McParland University of Toronto, Melanie Hammond Mobilio University of Toronto, Carol-Anne Moulton University of Toronto

Background/Purpose: Cognitive flow is a state in which individuals experience heightened awareness, performance, and reward. Although this state has been deeply explored and applied in elite sport and psychology, less is known about flow states in healthcare settings. This systematic review sought to summarize the information currently available on the phenomenon of flow in healthcare and to identify gaps in knowledge on this concept.

Methods: A systematic search using keywords related to cognitive flow, positive psychology, the zone, healthcare, and clinical practice was conducted. Two independent reviewers screened all articles and extracted data pertaining to study characteristics, 
study design, and psychometric properties. Data pertaining to discipline and salient results were noted.

Results: 4834 articles were initially identified; after duplicate removal, 4824 remained. 207 articles were included in full-text review after title and abstract screening. Overall, we found a paucity of literature on flow in healthcare settings. Many articles focused on workplace engagement and enjoyment. Common themes included the potential impacts of engaged and disengaged workers in healthcare, prevalence of burnout and stress in healthcare, the importance of work environment on work engagement, and the benefits of self-awareness and mindfulness in both work and learning.

Conclusion: Understanding flow states in healthcare may lead to new avenues for combating burnout, enhancing career satisfaction, and promoting wellness among providers. Future studies are needed to more deeply understand how flow is experienced in clinical settings, and how we might tailor training to help students achieve flow.

\section{DP 10-4 Spiralling Family Medicine Clerkship Curriculum to Enhance Learning using Both Asynchronous and Synchronous Modalities}

Keyna Bracken McMaster University, Parth Sharma McMaster University, Emily Allison McMaster University

Background/Purpose: The COMPASS curriculum was initiated at the McMaster DeGroote School of Medicine in 2006 with the tenets: concept-oriented, multidisciplinary, problem-based, practice for transfer, simulations in clerkship, and streaming. The practice to transfer component concerns the ability to transfer previous knowledge, applying it in a different context which is central in this innovative curricular project. The Family Medicine clerkship program developed a new E-learning platform by revisiting pre-clerkship Problem-based learning cases, adding layers of complexity to foster clinical knowledge translation in clerkship during which the cases are completed asynchronously and in synchronous tutorials.
Summary of the Innovation: Building on the foundation of curriculum development strategies and influence of adult learning theory, a Family Medicine integrated curriculum was designed by modifying preclerkship cases to have a practice to transfer focus. Students prepare for tutorial using the new Elearning modular platform. The modules integrate preclerkship and clerkship curriculum, including knowledge-check questions to promote selfassessment. The repetition of formative assessment aims to promote learner self-assessment and preparedness for the clinical decision making focused exit examination. The "super-tutorials" were designed to enhance interaction between clerks and a maestro (content expert), and the E-learning platform to enhance student engagement using polling questions and interactive features.

Conclusion: The development of asynchronous learning modules and synchronous learning opportunities through maestro-led "super-tutorials" allows for students to recall previous knowledge concepts and apply them in more complex situations thereby facilitating knowledge transfer. The maestro challenges this knowledge by guiding students through novel scenarios, which further develops knowledge transfer, and prepares students for diverse clinical scenarios.

DP 10-5 Internal Medicine Enrichment \& Development (IMED): early exposure to medicine subspecialties and its influence on students' perceptions of a career as an internist

Kaitlin Endres University of Ottawa, Katina Zheng University of Ottawa, Sarah Elias University of Ottawa, Mimi Deng University of Ottawa, Alexandra Kobza McMaster University, Aimee Li University of Ottawa

Background/Purpose: There are limited preresidency opportunities for students to experience the breadth of internal medicine (IM). IMED is a 2week program involving observerships, career talks and hands-on workshops in nine medicine subspecialties. Our objectives were to investigate whether the current IMED structure appropriately altered documented biases regarding a career in IM 
and which aspect of IMED students found most helpful in exploring these misconceptions.

Methods: Surveys were administered to three groups ( $n=16)$ : IMED participants who completed pre- and post-program surveys; "General control" participants who did not apply to IMED and "applied Control" participants who unsuccessfully applied. Scores were compared using Wilcoxon signed-rank and rank-sum tests for paired and unpaired data, respectively.

Results: $81 \%$ of participants reported a change in perception about the hours, work-life balance and job prospects as an internist, while $63 \%$ of participants reported a change in perception regarding procedural skill required. $81 \%$ of IMED participants reported that they were more likely to pursue IM following IMED. When students reported a change in their bias towards a career as an internist, $79 \%$ of the time this change was attributed to career talks. However, despite these self-reported results, there were no significant differences in misconception scores between groups.

Conclusion: IMED successfully improved preclerkship students' self-perceived understanding of a career as an internist. Career talks were the most valuable resource for students in altering misconceptions. In the future, our questionnaire needs adjusting. Participants felt that they had a change in their perception; however, due to the way questions were posed and scored, our objective data was not consistent with participants' opinions.

\section{DP 10-6 Redesigned conceptual mapping to} support self-regulated learning in problembased curriculum: from engineering to medical education

Veronique Foley Université de Sherbrooke, David Foley Université de Sherbrooke

Background/Purpose: Problem-based learning (PBL) has been recognized and used for decades in medical education to promote self-learning and clinical reasoning. Studying in a PBL curriculum requires selfregulated learning (SRL). This skill is not easily integrated, and students are lacking effective strategies to direct their own learning. Concepts mapping (CM) is a recognized strategy to support student SRL but several challenges exist. For students, $\mathrm{CM}$ may be a lengthy and strenuous process with difficulties combining and navigating thousands of concept maps. For educators, providing timely feedback can be a struggle. To address these issues, a software platform with strong cognitive foundations has been developed to support learning, collaboration and problem solving. The project stems from 8 years of research and developments in our engineering faculty and is being adapted to medical PBL program with promising results.

Summary of the Innovation: Relate! allows learners to organise knowledge, documents and references from all courses in one central place through innovative "extended concept maps". It is a collaborative platform that gives spaces for teams to build shared understanding, and it has a mechanism for teachers to evaluate and interact with students. Fifteen engineering students participated in qualitative interviews. They identified that it is a valuable tool to organise and support learning, and that it improves overview of the work compared to traditional $\mathrm{CM}$ and note books.

Conclusion: Preliminary results suggest that this app offer innovative and concrete means of supporting external feedback and scaffolding of knowledge. Application to medical education may be interesting as it is aligned with PBL, self-regulated learning and the need to integrate a growing body of knowledge.

\section{DP 11-1 "Get real"; promoting 'unstandardizable' real patients in OSCEs}

Grainne Kearney Queen's University, Belfast, Jenny Johnston Queen's University, Belfast, Nigel Hart Queen's University, Belfast, Gerry Gormley Queen's University, Belfast

Background/Purpose: Objective Structured Clinical Examinations (OSCES), dominant in health professions education (HPE) over the last forty years, seek to standardise assessment of and for practitioners. Critical research has expressed concern about the unintended but undesirable consequences of OSCEs. From 2023 the General Medical Council (GMC), the regulatory body for doctors in the United Kingdom (UK), will increase regulation in graduating assessments, to include OSCEs, bringing the UK in line 
with North America. This increased emphasis on objective, standardized and therefore accountable assessments creates difficulties for faculty keen to involve real patients.

Methods: This PhD used the method/theory of Institutional Ethnography, exploring increasing regulation into graduating OSCEs in a UK medical school. Data collection involved ethnographic observations, formal interviews, informal "chats" and the texts these people used. The aim was to explicate the work in OSCEs on the ground and in tandem, explore how and where this work was organized from.

Results: The need to standardize was a dominant thematic. I will present composite accounts of how students interacted with simulated patients in contrast to interactions between students and real patients in graduating OSCEs. These accounts allow differing emphases to be highlighted and depict what is lost in scripted interactions within regulationmandated accountability.

Conclusion: Real patients offer disruption to extreme standardization imposed through regulation; recalibration of thinking and commitment is required to promote unstandardized and unstandardizable person to person interactions. These 'non-insiders' push educators to question abstraction and obscurity in assessment and to focus on real clinical practice these students are about to embark on.

\section{DP 11-2 Minimal Criteria for Lung Ultrasonography}

Irene Ma University of Calgary, Janeve Desy University of Calgary, Vicki Noble Cleveland Medical Center, Andrew Liteplo Massachusetts General Hospital, Paul Olszynski University of Saskatchewan, Elaine Dumoulin University of Calgary, Shane Arishenkoff University of British Columbia, Renee Dversdal Oregon Health and Science University, Gigi Liu Johns Hopkins University, Brian Buchanan University of Alberta

Background/Purpose: Point of care ultrasonography (POCUS) is increasingly being incorporated into residency education. Despite this, there is no clear scanning standards, resulting in variable education being delivered across centres. This consensus study seeks to define the minimal criteria for all intended uses of lung POCUS for Canadian internal medicine training programs. Achieving consensus for minimal criteria will support standardization and enhance the overall quality of POCUS education.

Methods: Two independent investigators drafted a list of minimal criteria for lung POCUS, based upon a review of the literature. Minimal criteria refers to standards that a trainee must successfully demonstrate or achieve in order to be considered minimally acceptable. This draft criteria was then piloted as a survey on 14 internal medicine and family medicine physicians with at least 1 month of POCUS training. Suggestions were used to create a preliminary final criteria that was then surveyed to 10 international POCUS experts. Inclusion criteria for POCUS experts included the following: $\geq 1$ year of POCUS fellowship training or $\geq 3$ year experience in teaching and assessing POCUS and $\geq 3$ peer reviewed POCUS-related publications. Experts then completed 3 rounds of the 100-item online survey; consensus was defined as agreement by $70 \%$ or more.

Results: The response rate for the survey was $100 \%$ in all 3 rounds. In total 74 items met consensus for inclusion (51 in round 1, 15 in round 2 and 8 in round 3), 24 met consensus for exclusion (17 in round 1, 5 in round 2 and 2 in round 3 ), and 2 did not meet consensus.

Conclusion: In this study, we used consensus methods to achieve a 74-item minimal criteria standard for lung ultrasound in Canadian internal medicine training programs. This set of criteria will help standardize teaching, performance, and assessments, and ultimately enhance the quality of education in this evolving technique.

\section{DP 11-3 A Quality Improvement Initiative to} Improve Timeliness of Feedback

Sean Borle University of Alberta, Tracey Hillier University of Alberta, Susan Andrew University of Alberta, Cody Surgin University of Alberta, Hollis Lai University of Alberta

Background/Purpose: Medical schools must ensure the timeliness of clinical assessment feedback for students. This is problematic when programs are delivered across multiple sites with a large pool of 
instructors. A five year quality improvement program has been ongoing to address the timeliness of clinical assessments. The purpose of this study is to evaluate the effectiveness of this initiative.

Summary of the Innovation: This educational quality improvement initiative consisted of three parts. First, a data dashboard was developed to monitor the submission of assessment forms. Submissions were tracked to monitor the timeliness of feedback, relative to the midpoint and end point of each clerkship. Second, this dashboard was reviewed monthly by MD Program committees, such that all clerkships could see each other's progress. Third, a reminder process triggered follow-up with appropriate faculty when feedback from a rotation was late, potentially leading to non-compliance with program expectations. Over the past five years, 9878 assessment forms were tracked. A total of $0.8 \%$ of summative and $2.1 \%$ of formative assessment forms were found to be non-compliant with program expectations for timely submission.

Conclusion: Our project demonstrates the effectiveness of an electronic dashboard, combined with stakeholder buy-in and continuous monitoring, in facilitating compliance with the provision of timely feedback to students. Further research in this area will examine other methods of compliance encouragement which could build on the success of this project.

\section{DP 11-4 The Development and Validation of}

\section{a Simulated Competency Assessment in} Diabetic Wound Management

Omar Selim University of Toronto, Andrew Dueck University of Toronto, Catharine Walsh University of Toronto, Ryan Brydges University of Toronto, Mahan Kulasegaram University of Toronto, Allan Okrainec University of Toronto

Background/Purpose: Diabetic foot wounds comprise a third of diabetes-related healthcare expenditures, and are the primary cause of amputation in Canada. Few studies focus on how to teach and assess wound management. Given the importance of 'assessment for learning' in Competence by Design, we aimed to develop, and examine specific sources of validity evidence for, an assessment tool of wound management competencies.

Methods: We organized our tool development and validation process using Kane's framework. Using a nominal group process involving 9 Canadian experts in diabetic wound management, we developed the tool items, and two 10-minute simulation-based testing scenarios. We then assessed 74 participants' (61 physicians, 13 non-physicians) performance during the two scenarios: 44 novices (500). Two assessors independently rated participants using our tool. Reliability was evaluated using Generalizability Theory. Test-retest reliability was measured with intraclass correlation coefficient (ICC) comparing raters' scores across scenarios. We also compared performance scores across the three levels of experience

Results: We organized our tool development and validation process using Kane's framework. Using a nominal group process involving 9 Canadian experts in diabetic wound management, we developed the tool items, and two 10-minute simulation-based testing scenarios. We then assessed 74 participants' (61 physicians, 13 non-physicians) performance during the two scenarios: 44 novices (500). Two assessors independently rated participants using our tool. Reliability was evaluated using Generalizability Theory. Test-retest reliability was measured with intraclass correlation coefficient (ICC) comparing raters' scores across scenarios. We also compared performance scores across the three levels of experience

Conclusion: The accumulated validity evidence suggests our tool can be used to assess novice clinicians' competence in diabetic wound management during simulated cases. We plan to continue establishing validity evidence for use in other settings. 
DP $\quad 11-5 \quad$ Using Workplace-Based Assessments to Drive Post-Call Feedback: Can It Work?

Amy Lu University of Toronto, Adelle Atkinson University of Toronto, Julie Johnstone University of Toronto

Background/Purpose: Workplace-based assessment (WBA) is a critical component of competency-based medical education (CBME). Literature on WBA of performance during overnight call (e.g. post-call), however, is limited. We sought to evaluate a new post-call assessment tool for subspecialty overnight call within a paediatric residency.

Methods: Web-based surveys were sent to residents before tool implementation and monthly afterwards. Surveys explored frequency of actionable feedback provided and Likert-scaled opinions of tool usefulness in facilitating feedback (in CanMEDS Medical Expert [ME] and Manager/Leader [M/L] roles), feasibility within clinical workflows, preference for a longer (milestones specified) or shorter (assessment of entrustment, comments alone) tool, and qualitative comments. Quantitative data was summarized using descriptive statistics. Qualitative data was coded to identify themes.

Results: Response rates averaged 41\% (average monthly $n=12$ ). Median shifts with ME feedback increased 2 months post-tool (3/4 shifts worked, vs 1 pre-tool), but returned near baseline at 4 months. Shifts with $M / L$ feedback $(0-1.5 / 4$ vs 1$)$ was unchanged. Residents were divided between agreement (6/16) and disagreement (5/16) on the tool's usefulness facilitating ME feedback, and disagreed on its facilitating $M / L$ feedback (8/16) and feasibility within clinical workflows (10/16). Qualitative analysis revealed themes around nonactionable feedback; barriers to tool feasibility including handover structure, fellow availability and knowledge, interpersonal burden, and platform issues; and preference for shorter tools.

Conclusion: These findings identify key gaps in using WBA to meet CBME goals of increasing competencydeveloping feedback. Addressing gaps within feedback quality and factors influencing tool feasibility and logistics is essential for successful CBD implementation.

\section{DP 11-6 Using Kane's Validity Framework to establish evidence for locally Developed MMIs: Implications \& Interpretations}

Fern Juster McMaster University, Kelly Dore
McMaster University

Background/Purpose: The Multiple Mini Interview (MMI) has demonstrated validity evidence for personal competencies as indicated by the original publications' reliability values of 0.65 to 0.78 . More medical schools are developing their own local MMIs using specific competencies based on mission, values, population served, and applicant pool. It is essential that schools gather validity evidence when using high stakes assessments.

Methods: In a review of $10 \mathrm{MMI}$ papers, five cited reliability, ranging from 0.47 to 0.89 and none described undertaking a validation process. Kane's validity framework was applied to a locally developed $\mathrm{MMI}$ in the US to assess its evidence. The four inferences or phases, scoring, generalizability, extrapolation, and implication were used to identify validity threats and possible solutions.

Results: The threats to validity consisted of systematic error from rater variability and construct under-representation identified in the scoring and generalizability phases. Rater variability was managed with rater training and feedback. CUR was addressed by blueprinting scenarios and removing scenarios that were too difficult or too easy. The reliability of the MMI was 0.65 , indicating that the majority of the variance was attributable to the applicant. As real-world data is not available, the extrapolation inference was supported by content validity (matched to AAMC's recommendation) and convergent validity ( $r=0.40$ between $\mathrm{MMI}$ and CASPer).

Conclusion: Using a consistent validity framework will help developers/designers of a local MMI support admission decisions. It will also allow us to compare and contrast $\mathrm{MMI}$ variation to understand the critical components in $\mathrm{MMI}$ implementation in different contexts. 


\section{DP 11-7 Perceptions of Competency; does} gender influence self-assessment in UGME?

Helen Mawdsley University of Manitoba, Christen Rachul University of Manitoba, Keevin Bernstein University of Manitoba, Ira Ripstein University of Manitoba, Joanne Hamilton University of Manitoba

Background/Purpose: In 2010, the University of Manitoba MD program began a process of curriculum renewal. Implemented over the next five years, the new curriculum included substantial changes to both the format and content of the curricula. The Office of Educational and Faculty Development is conducting a multi-method evaluation of the curriculum. One question in the study is: Does an integrated, spiral curriculum change student's self-assessed learning? This presentation will explore the influence of gender on perceived competency of students at the end of clerkship, both before and after implementation of the new curriculum.

Methods: Students were asked to self-assess their performance on MD program curricular objectives at various points during their studies. Students rated their perceived competence on each of the MD program's learning objectives, using a paper survey and a five-point Likert scale. Data were analyzed with the Kruskall-Wallis test using SPSSv25.

Results: Statistically significant differences were found for a number of program objectives, with female students rating objectives related to the Medical Expert, Communicator, Collaborator, Health Advocate and Professional CanMEDs Roles higher, and males rating objectives related to the Scholar, and Manager higher.

Conclusion: There may be gender related differences in self-assessed competence for CanMEDs domains. These may have implications for exploring concepts such as imposter syndrome, clinical confidence, and gender-based differences in assessment of learning. Further study is needed to determine the influence of other factors, including student outcomes and admission variables, on self-assessed competence.

\section{DP 11-8 Un soutien innovateur pour les étudiants qui préparent leur EACMC-1}

Véronique Phan Université de Montréal, Stéphane Ouellet Université de Montréal, Dania Ramirez Université de Montréal, Josée Sabourin Université de Montréal, Isabelle Perreault Université de Montréal, Nicolas Fernandez Université de Montréal

Background/Purpose: L'Examen d'Aptitude du Conseil Médical Canadien (EACMC-1) requiert une préparation échelonnée sur plusieurs mois, années. Conscients des enjeux de cet examen, les étudiants éprouvent de l'anxiété durant la préparation ainsi qu'au moment de l'examen. Le Vice-Décanat de Premier Cycle de l'Université de Montréal cherche à réduire cette anxiété en offrant du soutien et des outils dans la préparation du EACMC.

Methods: En partenariat avec le Centre étudiant de soutien à la réussite, nous avons mené des groupes de discussion avec des résidents de première année ayant bien réussis le EACMC. Les discussions se sont concentrées sur les stratégies utilisées durant la préparation à l'examen et durant l'examen. Un groupe de discussion ainsi qu'un questionnaire a été envoyé à l'ensemble des externes qui s'apprêtent à faire l'examen, afin de connaître leur besoins lors de cette préparation.

Results: Durant la préparation à l'examen, les stratégies utilisées chez les résidents ayant réussis l'examen sont celles d'une gestion du temps efficace et d'une bonne utilisation des ressources disponibles. Le développement d'une fine connaissance de leur profil d'apprentissage en adaptant leur calendrier, la matière à réviser et les ressources utilisées mène au succès. Ils s'assurent de créer les conditions idéales pour étudier (seul et/ou en groupe, etc.), d'avoir les outils nécessaires (livres, et banque de questions de pratique) et de conserver une vie équilibrée (sommeil, alimentation et loisir).

Conclusion: La mise en évidence de stratégies efficaces et le profil d'apprentissage de l'étudiant pourrait contribuer à la diminution de l'anxiété lors de la préparation du EACMC. 
DP 11-9 Performance Report for a 10-YearOld MD/PhD Program: a Survey of Trainees at the University of Ottawa

Adam Pietrobon University of Ottawa, Lucia Chehadé University of Ottawa, Alexandra Beaudry-Richard University of Ottawa, Brian Keller University of Ottawa, Michael Schlossmacher University of Ottawa

Background/Purpose: MD/PhD programs are relatively new in Canada and are a key route to training the next generation of clinician-scientists. However, MD/PhD programs vary substantially by structure, funding and mentorship opportunities, and there exists a paucity of data on the overall student successes and challenges. The goal of this study is to assess objective and subjective metrics of the $\mathrm{MD} / \mathrm{PhD}$ program at the University of Ottawa.

Methods: Students in all cohorts were invited to complete a 58-question survey, and the resulting data were subsequently analyzed by descriptive statistics.

Results: On recruitment: Tuition support was considered an essential element in accepting the admission offer, as $47.8 \%$ of students would have declined admission without full UGME tuition coverage. On graduate training: The vast majority of respondents (78\%) expressed interest in taking graduate courses designed specifically for clinicianscientist trainees, which are currently absent. Regarding scientific activities: MD/PhD students were heavily engaged in scientific activities, with an average of 8.3 presentations/publications per respondent. On general challenges: Respondents indicated low satisfaction with formal career planning advice (28.6\% satisfied/very satisfied) and program transition guidance (22.2\%), whereas when delivered informally by peers both were experienced as more satisfying (65.2\% and $63.6 \%$ respectively).

Conclusion: This survey contributes to the growing body of knowledge surrounding the successes and challenges of $\mathrm{MD} / \mathrm{PhD}$ programs in Canada. The survey template employed is being shared to guide assessments at other institutions. This work is currently in submission at CMAJ Open.
DP 11-10 Field Notes: Effectiveness and Impact on Family Medicine Residents' Learning in Manitoba

Nicole Zaki University of Manitoba, Teresa Cavett University of Manitoba, Gayle Halas University of Manitoba

Background/Purpose: Field notes (FNs) are used in Family Medicine (FM) residency programs to foster reflective learning and facilitate feedback. Residents document dimensions of one clinical encounter, assess their strengths and weaknesses, and develop action plans to effect a change in practice. This study explored the use of FNs in the University of Manitoba's FM residency program.

Methods: This multi-method study examined 520 FNs from 16 recent graduates. Quantitative analysis (frequencies and means) enabled descriptions and comparisons between residency training sites; four themes emerged from inductive content analysis.

Results: Residents displayed cyclical variation in the generation of FNs, with peaks coinciding with the start of core FM rotations. The most frequently described Priority Topics were Skin Disorders, Infections, Depression, and pain management. All but 8 of the 99 Priority Topics (addressing complex psychosocial issues) were explored in this data set. Few FNs addressed the domains of Care of First Nations, Inuit, and Metis, Care of the Vulnerable and Underserved, and Behavioural Medicine and the CanMEDS-FM roles of FM - Procedural Skill, Leader/Manager, and Professional. Four themes (Patient-Centered Care, Patient Safety, Achieving Balance, and Confidence) were identified from narrative notes.

Conclusion: Vygotsky's Sociocultural Theory of Cognitive Development was proposed as a lens through which to examine factors influencing resident learning. Residents' discomfort with certain topics may be reflected in their avoidance of reflecting upon certain competencies in FNs thus impacting skill acquisition. Further research should explore factors influencing residents' selection of FN topics and how to best assist residents in becoming competent, confident practitioners. 
DP 12-1 Discordance Between CompetencyBased Assessment Using a Global Versus

Reductionist Approach for Medical

\section{Students}

Holly Caretta-Weyer Stanford University School of Medicine

Background/Purpose: The advent of competencybased education has led to concerns regarding reductionism in the assessment of clinical competence. This apprehension stems from a frequently utilized, fractionated approach using the assessment of isolated competencies to build a complete picture of clinical competence. In contrast, others argue that the EPA framework complements the construct of competencies, as EPAs describe a unit of work and require a global, holistic approach to the assessment of the various activities of a physician.

Methods: We designed a simulation-based workshop during our medical school's Transitions to Residency Course to discern whether the assessment of separate competencies to build a picture of clinical competence is equivalent to the global assessment of EPAs. Each student was assessed using individual competencies mapped to the Core EPAs, a modified supervision scale, and a global statement regarding entrustment and readiness for residency. These assessments were then compared to aggregate workplace-based assessment data on the various individual competencies from the core clerkships as rated by trained assessors and subsequently mapped to each EPA.

Results: Assessment data obtained during the Transitions to Residency course using the individual competencies from the EPA toolkits were highly correlated with the assessment of individual competencies obtained from ratings by qualified assessors in the workplace as part of the core clerkships. However, these individual competencybased assessments did not correlate with holistic, EPA-based global supervision scale ratings, entrustment decisions, or perceived readiness for residency.

Conclusion: The global assessment of EPAs and the judgement of entrustment appears to be a separate process from aggregating the assessment of individual competencies for raters. This may reflect variations in the approach to global assessment when compared to the assessment of individual competencies as well as the need to consider the construct of trustworthiness in addition to the learner's ability to perform the activities of a physician essential to succeeding in residency during the assessment process.

\section{DP 12-2 Perceptions of and Barriers to} Competency-Based Education

Lindsay Crawford Queen's University, Sean Taylor Queen's University, Nicholas Cofie Queen's University, Damon Dagnone Queen's University, Laura McEwen Queen's University

Background/Purpose: Competency-based education (CBE) has been implemented across Canadian postgraduate medical training programs through Competence By Design (CBD). We describe our initial experiences, highlighting perceptions and barriers to facilitate implementation at other centers.

Methods: An anonymous online survey was administered to faculty and residents transitioning to CBE (138 respondents) including 1) Queen's Residents (QR)[n=102], 2) Queen's Faculty (Program Directors and CBME Leads) [ $n=27$ ]and 3) Canadian Neurology Program Directors (NPD) $[n=9]$ and analyzed the data using descriptive and inferential statistical techniques.

Results: Perceptions were favorable $(\bar{x}=3.55, S D=$ 0.71 ) and $81.58 \%$ perceived CBE enhanced training; however, perceptions were more favorable in faculty. QF indicated that CBE did not improve their ability to provide negative feedback. NPDs did not perceive their institution had adequately prepared them. QR did not perceive improved quality of feedback. There was variability in barriers perceived across groups. NPDs were concerned about access to information technology. QF were concerned about resident initiative. QR felt assessment selection and faculty responsiveness to feedback were barriers.

Conclusion: Our results indicate Faculty were concerned about reluctance of residents to actively participate in CBE. Residents were hesitant to assume such a role due to lack of familiarity and perceived benefit. This discrepancy indicates attention should 
be devoted to: 1) institutional administrative/educational supports, 2) faculty development around feedback/assessment and 3) resident engagement to foster ownership of their learning and familiarity with CBE.

\section{DP 12-3 Development Of a Competency -} Based course for Pre-Cerlkship

Matt Kushneriuk University of Saskatchewan, Deirdre Andres University of Saskatchewan, Andrea Symon University of Saskatchewan, Di Naidu Naidu University of Saskatchewan, Joshua LLoyd University of Saskatchewan, Andrea Symon University of Saskatchewan

Background/Purpose: Competency based medical education (CBME) is becoming the norm in postgraduate and clerkship education. To support this transition in clerkship, we identified an opportunity in our pre-clerkship Clinical Integration courses for initial introduction of a competencybased educational model.

Summary of the Innovation: Clinical Integration is a set of courses over the first two years of medical school. The focus within these courses is on clinical reasoning, integration of clinical information, ethiclegal reasoning and information literacy. These skills lend themselves well to a competency-based teaching, learning and assessment model. In this report, we describe the processes used to develop this targeted curriculum including competency identification, competency levels/definitions, assessment, and evaluation strategies. Student focus groups, faculty consultation and review of existing literature were all employed in this iterative process. Implementation of $\mathrm{CBME}$ has required close collaboration between the course development team and information technology, assessment, and administrative faculty/staff.

Conclusion: This multistep iterative process has been successful in the development of a pre-clerkship competency-based curriculum. Students, faculty and staff have shown enthusiasm for implementing these changes. Both expected and unexpected challenges required attention during the development stages and inaugural implementation.
DP 12-4 Perceptions of academic day teaching and barriers to emergency medicine senior residents' attendance

Lisa Thurgur University of Ottawa, Miguel CortelLeBlanc University of Ottawa, Jeffrey Landreville University of Ottawa

Background/Purpose: Royal College Emergency Medicine (EM) trainees at the University of Ottawa participate in weekly Academic Full Days (AFD) that consist of didactic activities, simulation-based learning, and core content sessions referred to as Core Rounds (CR). Despite CR being intentioned for all EM trainees, an attendance attrition has been noted as trainees progress towards their senior (SR) years (PGY3-5). The objectives of this study were to (1) identify barriers to SR trainee CR attendance and (2) identify areas for CR improvement .

Methods: An on-line survey was administered to SR EM trainees (PGY3-5, $n=28$ ) and recent graduates from our program (practice year $1-2, n=20$ ) to explore perceptions of the value of AFDs, CR attendance barriers, and areas for CR improvement. The survey consisted of 5-point Likert scales and freetext responses. Quantitative responses were analyzed using Microsoft Excel. Free-text responses were analyzed qualitatively using thematic analysis. Each free-text response was reviewed independently by two investigators (JML, MCL) and underwent lineby-line coding. Through joint discussions, the codes from each response were synthesized and themes were identified.

Results: Of the 48 trainees and attendings surveyed, 32 responded (response rate 67\%). Most respondents (90\%) stated they benefited from SR trainee attendance when they were at a junior (JR) level. The majority perceived they benefited less from $C R$ as a SR trainee compared to when they were a JR trainee (85\%). Further, $87 \%$ responded that CR were not tailored to a SR level, and that they would attend more frequently if sessions were geared to their level (81\%). From our thematic analysis, three themes emerged relating to SR trainee absenteeism: 1) CR quality, 2) External Factors (eg. trainee fatigue) and 3) Misalignment with trainees' own education plan. We also identified three themes relating to areas for $C R$ 
improvement: 1) CR content, 2) CR format and 3) SR trainee involvement.

Conclusion: Respondents indicated a benefit to having SR trainee presence at CR. This study identified barriers to SR resident attendance at CR and areas for improvement. With the transition to competency based medical education it is critical that trainees engage in effective educational experiences, especially as the RCPSC does not mandate AFDs for EM training in this new curriculum. A culture-change initiative and $C R$ reformat is now underway at our institution with planned post-implementation analysis.

\section{DP 12-5 How do healthcare professions develop competency frameworks?}

Alan Batt Monash University, Australia, Walter Tavares University of Toronto, Brett Williams Monash University

Background/Purpose: Competency frameworks serve many roles including outlining characteristics of a competent workforce, facilitating professional mobility, and structuring curriculum and assessment. Given their significance in health professions, we sought to understand the strategies used in the development of existing competency frameworks.

Methods: We conducted a scoping review using the Arksey and O'Malley framework. Six electronic databases and three grey literature sources were searched using keywords related to competency frameworks. Studies of all types were included that described the development of a competency framework in a healthcare profession. Studies were screened for inclusion independently by two reviewers. Data synthesis was both quantitative and qualitative.

Results: 190 citations were selected for analysis. The majority of studies were conducted in medicine and nursing. We found a significant degree of diversity in methodological strategies, and inconsistent adherence to existing (albeit limited) guidance on framework development. Based on the variation we observed in choice, sequence, application and reporting of methods and strategies, there is no apparent standardised approach to competency framework development
Conclusion: Significant variation exists in the processes used to develop competency frameworks. Adherence to existing guidelines is inconsistent. This raises questions about which processes are fit for purpose and whether resulting competency frameworks capture the complexities associated with clinical practice, the value or completed frameworks to the corresponding profession, and whether existing processes lead to unintended but legitimized artificial outcomes. Guidance in competency development processes may need to be revisited

\section{DP 12-6 Development of Collaborative} Student \& UME Course Evaluation

Wenxuan Wang Western University, Michele Weir Western University, Kyle Massey Western University, Gary Tithecott Western University

Background/Purpose: Formal program evaluation is a critical tool for quality improvement (QI) in Canadian medical school curricula and CACMS accreditation requirements. Our school recently underwent curriculum renewal to a competencybased medical education (CBME) model in our undergraduate medical education (UME) program, which created the need for updated program evaluation that accommodates student feedback with UME central evaluation. We identified challenges in the redundancy and volume of evaluation surveys which resulted in student survey fatigue, lower response rates and less meaningful data. Although there is literature on curricular evaluation methods, there was sparse guidance on how to balance student feedback with central evaluation. Our initiative may be of interest to those implementing an evaluation approach which is collaborative between students \& UME.

Summary of the Innovation: Our former course evaluation consisted of two parallel processes: formal evaluations through UME, and independent studentrun evaluations which were not validated for central course evaluations. We merged the parallel streams into a central process with UME and student academic leads collaborating on evaluation instrument development, data analysis, and sharing of data with course leads and UME committees. 
Conclusion: As part of our commitment to innovative education and the new CBME program, we designed a collaborative program evaluation process to meet the demands of UME central program evaluation while incorporating student involvement. This resulted in fewer evaluation forms for distribution, streamlined the evaluation process, and allowed for collaboration between central UME \& students.

\section{DP 12-7 The effect of "active retrieval" on the learning of procedural skills}

Cyrus Hamadi Western University, Silvio Ndoja Western University, Charles Dion Western University, Alexander McCarton Western University, Brynn Charron Western University, Marie-Eve LeBel Western University

Background/Purpose: "Retrieval" allows to recover/access stored knowledge: it improves the learning. "Active retrieval" allows to retrieve knowledge through activities like answering questions, writing/talking about the new knowledge, trying to recall through discussions, etc. This method has not been studied in the surgical education setting. The purpose of this study was to evaluate if "active retrieval" through writing can improve procedural learning for simple fracture fixation versus "passive learning" only from reading. We believe active retrieval can improve long term retention of surgical skills.

Methods: In this pilot study, we randomized 50 preclerkship students to two groups: passive learning group or active retrieval group. All participants watched a video of an open reduction internal fixation with lag screw and plate/screws on Sawbones $^{\mathrm{TM}}$. Participants then performed the procedure under guided supervision. Next, they had 15 minutes to either read the steps (passive learning), or write down the steps from memory (active retrieval group). After a 5-minute washout period, participants performed again the procedure without any guidance (immediate assessment). Participants were brought a week later to perform the procedure without guidance (delayed assessment). Each performance was video-recorded for data analysis purposes.
Results: Both groups were similar in participants' age or gender. Participants in the passive learning group performed better at the immediate assessment phase. Participants in the active retrieval group performed better in the delayed assessment. There was a decrease in retention between the immediate assessment and delayed assessment for both groups.

Conclusion: Active retrieval appears to enhance long term retention of procedural learning.

\section{DP 12-8 Utility and feasibility of video-} based assessment for ureteroscopy and laser lithotripsy

Yuding Wang McMaster University, Kelly dore McMaster University, Edward Matsumoto McMaster University, Dana Russle McMaster University, Nathan Wong MSKCC, Jen Hoogenes McMaster University, Bobby Shayegan McMaster University

Background/Purpose: Assessment plays a central role in CBME. The operating room (OR) is a demanding environment with competing priorities placed on the primary surgeon, including the assessment of resident's performance. Previous studies show that assessment reliability decreases with increased task complexity. The objective of this study was to explore the utility of video-based assessment of resident performance in the OR.

Methods: 5 surgery cases performed by residents under staff surgeon guidance were captured on video using a 3-camera setup: the surgeon's view; the endoscopic view; and a 360-degree view of the OR. Following the case, the in-room staff surgeon assessed the resident using the 11-item entrustmentbased O-SCORE, and evaluated his or her own cognitive load during the case using the Surgical Task Load Index (SURG-TLX). Two independent staff surgeons later independently rated the videos to compare to the case surgeon. A post-assessment survey and interview were conducted with the case surgeon, resident \& video raters.

Results: The interrater reliability between video assessors was high $(\mathrm{k}=0.82)$ and mean O-Scores ratings between intraoperative and video assessors O-Score agreement were also high ( $r=0.7)$. O-SCORE domains for intraoperative performance had high agreement between the intraoperative assessment 
and video-based assessment ( $r=0.68)$. All assessors expressed low cognitive load during viewing or while scrubbed into the case with mean SURG-TLX scores of 5 (SD \pm 1.8 ). Video-based reviewers frequently rewound the video during critical steps of each case to assess resident performance.

Conclusion: This study showed that video-based assessment of resident performance during surgery using the O-SCORE is useful and feasible, with high interrater reliability among the case surgeon and independent video reviewers. Use of the SURG-TLX also provided new insight into the cognitive load of both case surgeons and video reviewers.

\section{DP 13-1 The Effects of Duty Hours on} Wellness Among Resident Physicians in

\section{Canada - A Systematic Review}

Miranda Wan University of Calgary, Stewart Spence University of Ottawa, Melissa Wan University of British Columbia

Background/Purpose: Resident duty hours (RDH) have emerged as a controversial topic in medicine, particularly given the introduction of night-float (NF) systems. However, limited evidence exists investigating the impact of RDH on well-being. We conducted a systematic review to better understand the effects of RDH on wellness among resident physicians in Canada.

Methods: A systematic review of duty hours on resident physician wellness in Canada was conducted using the PubMed, MEDLINE, and CENTRAL databases from 1989 to 2019. Literature screening and data extraction were independently conducted by two reviewers. Sub-analyses of the effects of RDH on wellness based on specialty and NF models were then performed. An explorative analysis was subsequently completed to identify themes related to resident perceptions of wellness based on duty hours.

Results: After screening 261 articles, a total of 14 articles met inclusion criteria. Nine articles (64.3\%) found a positive association between fewer RDH and improved wellness, while $3(21.4 \%)$ found no change, and $2(14.3 \%)$ found a decrease in wellness. Subgroup analysis of NF systems $(n=9)$ demonstrated a positive impact on wellness in 6 articles (66.7\%), no difference in 2 (22.2\%), and a negative impact in 1
(11.1\%). Sub-group analysis of specialty demonstrated a positive impact of fewer RDH on wellness among surgical residents in $71.4 \%$ of studies. Several themes emerged impacting residents perceptions of RDH on wellness, including: 1) flexibility in scheduling; 2) maximizing educational opportunities; and 3) changes in team dynamics.

Conclusion: Overall, RDH reforms improved resident well-being, however this relationship is complex. Further research is necessary to characterize the effects of factors such as specialty and system design on resident wellness.

\section{DP 13-2 Reflection on Professional Identity: A Novel Way to Support Resident Wellness}

Carly Schenker University of Toronto, Lindsay Herzog University of Toronto, Erin Bearss University of Toronto, Milena Forte University of Toronto, Michael Roberts University of Toronto, Ian Waters University of Toronto, Diana Toubassi University of Toronto

Background/Purpose: The prevalence of burnout, depression, and anxiety amongst medical trainees is worryingly high, with consequences including reduced learner productivity, poorer quality of care, increased medical errors, and decreased empathy. Interventions that have been shown to mitigate these effects have often included some form of reflective practice, although none have emphasized the role of professional identity formation, a process which is increasingly recognized as critical to the maintenance of wellness. To address this gap, a novel curriculum was designed to support Family Medicine resident wellness through reflection on professional identity.

Summary of the Innovation: All Family Medicine residents (total 50) at two academic Family Medicine Teaching Units at the University of Toronto participated. Four faculty members were recruited to facilitate. The curriculum included eight 2-hour reflection sessions occurring periodically through the two-year residency. Residents were divided into small groups of approximately 12 , based on residency year and training site. Sessions were facilitated by faculty members from the alternate site. Qualitative data was collected through post-session feedback forms (administered after each session), as well as through resident and faculty focus groups (which probed for 
more general impressions at the end of the academic year).

Conclusion: Thematic analysis demonstrated that a longitudinal curriculum encouraging reflection on professional identity is highly valued. Additional findings regarding the importance of "protecting" the discussions, facilitator skillset, and the role of individual personality will be used to guide the curriculum's future iterations.

DP 13-3 Teaching mindfulness-based stress management techniques to medical students: Pilot results from the Simulated Training for Resilience in Various Environments (STRIVE) program

Stephanie Smith University of Calgary, Lauren Griggs University of Calgary, Franco Rizzuti University of Calgary, Joan Horton University of Calgary, Aliya Kassam University of Calgary, Allison Brown University of Calgary

Background/Purpose: The prevalence of burnout raises many concerns with regards to organizational culture and patient safety. Difficult clinical events during medical school (e.g., patient death, moral distress) may negatively impact resiliency and wellbeing among medical students. Previous studies suggest that resiliency training surrounding stressful clinical events during medical school may alleviate the potential impact of these encounters (Houpy et al, 2017)

Methods: To prepare current and future health professionals in mindfulness-based stress management tactics, the Simulated Training for Resilience in Various Environments (STRIVE) program was developed to train medical learners about the Mental Health Continuum, the Big Four + , and to provide a safe, non-judgemental learning environment to practice these skills in realistic, simulated clinical scenarios. A longitudinal design examined the impact of STRIVE tactics on medical students following the initial pilot in Summer 2018.

Results: Increases in participant's confidence to identify early signs of burnout, support peers, find resources, and teach peers about resiliency techniques increased post-STRIVE $(16.9+/-1.71)$ and were sustained 6-months following the program $(14.87+/-2.07)[F(2,97)=23.964, p=0.000]$. Mean increases on the CD-RISC following STRIVE (15.9 +/2.09) as well as 6-months later $(15.8+/-2.19)$ were not statistically significant $[F(2,97)=1.082, p=0.343]$.

Conclusion: Mindfulness-based stress management tactics may be beneficial for medical students to learn to promote student confidence and skills in identifying signs of burnout, support peers, find resources, and teach others. Future research on how to sustain improvements in self-reported resiliency in medical education is warranted.

\section{DP 13-4 Harnessing the power of positive language to promote physician well-being}

Marie Leung McMaster University, Olivia Geen McMaster University, Samantha Woodrow Mullett Memorial University of Newfoundland, Meirui Li Western University, Naomi Mudachi University of Toronto, Hely Shah University of Alberta

Background/Purpose: Physician burnout has been acknowledged as an issue of increasing importance. To combat burnout, the promotion of physician wellbeing has previously focused on individual interventions, however, increasing emphasis is being placed on cultural changes in medical education and the health care system. The relationship between language and culture has been well described in literature for other fields of study, including anthropology, philosophy, linguistics and psychology. The purpose of this project was to determine the impact of language in promoting a culture of wellness. To our knowledge, this is the first innovation directed at harnessing positive language at multiple levels of medical training in order to systemically promote physician well-being.

Summary of the Innovation: A pro-wellness language task force was created by Resident Doctors of Canada, with representation from multiple subspecialties including internal medicine, pathology, and psychiatry. A literature search was conducted looking for evidence on the use of language to promote cultural change in medicine and other professions including nursing and business. Through an iterative process, themes were derived on words $\&$ phrases that promoted wellness, or 'pro-wellness' 
language: (1) validation of stressful situations (2) creating a sense of solidarity and (3) supporting peers in their pursuits for wellness. Two infographics were created to disseminate these themes. These infographics will be shared with medical trainees to promote reflection on the impact of language on promoting wellness in daily conversation.

Conclusion: Language has a strong impact on shaping culture in medicine through validation, creating solidarity and support among physicians. Further dissemination of 'pro-wellness' language is important for medical trainees, residents and supervising physicians in order to promote a culture of wellness.

DP $13-5$ The non-medical use of
prescription psychostimulants among
medical students of Quebec

Roxanne St-Pierre-Alain Université de Sherbrooke, Maxime Couture Université Laval, Audrey Désilets Université de Montréal

Background/Purpose: Few data are currently available on the non-medical use of prescription psychostimulants in Quebec among medical students. The FMEQ has therefore examined the situation to better understand this issue that affects many of its members.

Methods: An optional survey was distributed in the fall of 2018 to medical students in Quebec through local student associations and the FMEQ, particularly through e-mails and social networks.

Results: In total, the survey collected 884 responses, representing $21 \%$ of the medical student population in Quebec. $71 \%$ of respondents self identified as women, $28 \%$ as males and $6 \%$ of participants reported having a diagnosis of ADHD. $8 \%$ of respondents reported having used psychostimulants for non-medical purposes. Also, $1 / 3$ respondents have already considered using psychostimulants during their undergraded medical studies without having done so.

Conclusion: The survey results reveal that the proportion of medical students in Quebec who have used psychostimulants for non-medical purposes $(8 \%)$ is above the average of the general student population in Canada (4.5\%), but below the use of
American medical students (10.1\%). The survey also reveals that the majority of students who take nonprescribed stimulants do not do so with a purely competitive aim; curiosity, delay and self-medication being equally prevalent. Awareness campaigns including side effects would be beneficial for this student population to prevent abuses.

\section{DP 13-6 Evaluating and reinforcing medical students' engagement with peer-run and} faculty-led wellness initiatives

Valerie S. Kim University of Toronto, Allison J. Chen University of Toronto, Anna Chen University of Toronto, Lauren A. Beck University of Toronto, Ivona Berger, University of Toronto, Natalie Phung, University of Toronto, Caroline Park, University of Toronto, Lisa Vi, University of Toronto

Background/Purpose: Committed to promoting wellness within University of Toronto's MD program, Student Health Initiatives and Education (SHINE) organizes a range of events for academic development, career exploration, and resilient selfcare. In collaboration with the Office of Health Professions Student Affairs (OHPSA), we aim to design opportunities that can best support mental health throughout medical training. This vision necessitates evaluation of experiences with wellness initiatives and exploration of concerns, barriers, and facilitators for student engagement.

Methods: A survey invited students to share reasons for or against engaging with the events and services offered by SHINE and OHPSA, thoughts on whether and how such programs contribute to their wellbeing, and ideas for alternative supports.

Results: Thematic analysis of survey responses $(n=69)$ revealed a prevailing positive attitude towards student-run wellness initiatives. In particular, the events with the highest attendance and engagement focused on relieving stress with a sense of solidarity and fostering dialogue on the challenges of work-life balance in medicine. Perceived facilitators to greater participation emphasized convenience, namely with regards to location and time. Interestingly, the latter was reiterated as a key concern for OHPSA's programs, with scheduling difficulties considered the predominant barrier to seeking professional services. 
Accordingly, the survey respondents advocated for strengthening SHINE's collaboration with faculty, such that both student- and staff-led supports become more accessible.

Conclusion: Receptivity to wellness initiatives is dependent on their ability to address student concerns. By collecting and incorporating feedback, peer-run and faculty-led groups can develop accessible opportunities tailored to medical students' needs, thereby strengthening their engagement and well-being.

\section{DP 13-7 Are the Wellness Benefits of Mindfulness and Resilience Limited by Hidden Motivational Factors in the Learning Environment?}

Adam Neufeld University of Saskatchewan, Greg Malin University of Saskatchewan

Background/Purpose: The learning environment in medical school is an important consideration for student motivation and wellbeing. The purpose of this work is threefold: a) to explore how student mindfulness and resilience predict changes in their perceived stress and coping; $b$ ) to assess how this relationship might change, accounting for students' perceptions of the supportiveness of their learning environment to their basic needs of autonomy, competence, and relatedness; and c) to assess whether perceived stress and coping vary by gender and year of study.

Methods: The authors conducted an online survey at the University of Saskatchewan, measuring student mindfulness and resilience, perceived stress, coping, and perceived need satisfaction/frustration. Hierarchical regression assessed how gender, year, mindfulness, and resilience predicted changes in perceived stress and coping, accounting last for students' perceived need support.

Results: 197/400 (49\%) students were evaluated: 92 males (47\%), 105 females (53\%) - 71 (36\%) 1st years, $58(29 \%)$ 2nd years, $36(18 \%) 3 r d$ and 4th years. Higher mindfulness, resilience, and perceived need satisfaction predicted lower perceived stress, more adaptive, and less maladaptive coping. When students perceived their learning environment as need-frustrating, it predicted the opposite effect, and neither mindfulness nor resilience suppressed maladaptive coping. Third years reported the highest stress and use of denial. Females reported coping with more instrumental and emotional support and behavioural disengagement.

Conclusion: Mindfulness and resilience may be beneficial to medical student stress and coping; however, their wellness benefits likely depend on motivational factors within the learning environment (i.e. how supported students feel their basic needs are).

\section{DP 13-8 Sentinel Resident Project}

Laurence Labine Université de Montréal, Maude Cigna Université de Montréal, Ariane DeneaultMarchand Université de Montréal, Karina Salvo Labelle Université de Montréal, Gabriel Lavoie Université de Montréal, Sarah-Émilie Racine-Hamel Université de Montréal, Sophie Marcoux Université de Montréal

Background/Purpose: Physician residents' well-being is a persistent yet evolving challenge. Difficult learning environments often result in psychological distress and can lead to mental illness. According to a recent survey conducted by the "Fédération Médicale des Résidents du Québec" (FMRQ) based on the Maslach Burnout Inventory (MBI), 55\% of residents were suffering of burnout. Furthermore, $27 \%$ of residents admitted having suicidal thoughts at least once during their lifetime in a study performed by the Canadian Medical Association (CMA) in October 2018. Despite available resources, most residents remain unaware of their existence or are reluctant to consult them.

Summary of the Innovation: To address this important issue, we created a program called Sentinel Resident. The main objective consists of on site, peerled support through careful listening and referral to adequate resources. During our pilot project, 20 residents among 9 medical and surgical specialities were selected by their peers or volunteered to assume these responsibilities. They are currently distributed in 10 different training sites. Residents received small-group training with Quebec Physician Health Program' instructors. A simulation and 
feedback approach was used to learn how to recognize and help colleagues in distress. A guide was also created to assist sentinel residents. It includes references for different problematics: financial, parental difficulties, harassment, substance addiction etc.

Conclusion: This program aims to rapidly identify and help residents in emotional need by providing on site peer support. The ultimate goal is to improve their well-being by ensuring a supportive, non-judgmental climate and rapid redirection to the right resources in time of need.

DP 13-9 Harassment Reporting Mechanisms for Medical Students, Residents and Physicians in Calgary, Alberta: An Environmental Scan

Kendra Martel University of Calgary, Shannon Ruzycki University of Calgary

Background/Purpose: Harassment has been welldocumented in medical training and the physician workplace; current best practices for reporting are described (1-5). Despite increasing recognition of its burden, little has been reported about the available mechanisms for reporting harassment for physicians and trainees. We performed an environmental scan of harassment reporting mechanisms for medical students, residents, and physicians working in Calgary, Alberta in order to: (1) produce a guide that medical professionals and trainees can utilize if they experience harassment in the workplace, and (2) identify potential limitations or gaps in harassment reporting mechanisms that create barriers to reporting.

Methods: A list of reporting mechanisms was created through a detailed internet search of publicly available mechanisms and snowball sampling. A standardized data collection template was created based on known best practices for harassment reporting (5). Each organization was contacted by email or telephone and interviewed using the standardized data collection template during August 2019.

Results: We gathered valuable insights regarding the complexity and lack of transparency and communication between reporting mechanisms in Calgary. The numerous organizations to which medical professionals and trainees may file harassment reports in Calgary creates a lack of direction for individuals. Second, many reporting mechanisms refer individuals to other agencies, rather than pursuing direct involvement. Lastly, lack of communication between reporting policies raises the possibility of multiple reports against the same individual without identifying repeat offenders.

Conclusion: This research identifies a need for a single regulated domain to which all reports can be filed and reviewed to adequately address physician and learner harassment.

\section{DP 14-1 "BEEP-BEEP SIM": Gamified On-Call} Simulation Curriculum for Undergraduate Medical Education

Anthony Seto University of Calgary, William Kennedy University of Calgary, Mackenzie Margetts University of Calgary, Mitchell Rohatensky University of Calgary, Jian Choo University of Calgary, Sean Crooks University of Calgary, Nicole Ertl University of Calgary, Nazia Sharfuddin University of Calgary, Nathan Zondervan University of Calgary, Lucas Streith University of Calgary

Background/Purpose: "BEEP-BEEP SIM" prepares medical students to be paged for on-call situations. It combines escape game elements and low-fidelity simulation to achieve learning for tailored objectives, drawing on teaching strategies of gamification and repetition, and enhancing motivation through the self-determination theory (through an experience that promotes competence, autonomy, and relatedness).

Summary of the Innovation: 5 teams of 3-5 students $(n=20)$ piloted "BEEP-BEEP SIM". Teams completed 6 "rooms" in any order. Each room contained 4 puzzles regarding on-call clinical topics. If teams exceeded 10minutes for a room, a facilitator assisted. Solving 4 puzzles enabled unlocking of a room's box. Each box contained a summary card of teaching points for that room, which students reviewed. Once 6 rooms were completed, students were debriefed; this included a verbal quiz to promote retrieval of learned content and an instructor-facilitated summary to reiterate 
learning points. The average learner-reported confidence $(n=20)$ in learning objectives for Hyperkalemia (2.24/5.00 to $4.02 / 5.00)$, Chest Pain (3.46/5.00 to 4.46/5.00), Hyperglycemia (2.86/5.00 to $3.98 / 5.00)$, Agitation (2.39/5.00 to $4.34 / 5.00)$, Fall (2.81/5.00 to $4.14 / 5.00)$, and Fever $(2.81 / 5.00$ to $3.96 / 5.00$ ) increased pre- vs post-session (all $p<0.001$, 1-tail repeated-measures t-test). "BEEP-BEEP SIM" was reported as "fun", "interactive", "engaging", "low stakes", and "high-yield". On a scale of "not useful" (0) to "very useful" (2), students $(n=19)$ rated gamification $1.89 / 2.00$ and the 4-phase repetition design 1.92/2.00.

Conclusion: "BEEP-BEEP SIM" improves learner confidence, with advantages of being relatively lowcost and portable. This educational model may be adapted to train learners within any field, and have interdisciplinary potential, as objectives and associated puzzles are easily customizable.

\section{DP 14-2 Application of novel high-fidelity} simulation-based tools for the training of digital rectal examination

Louise Hufton Croydon University Hospital, Alejandro Granados, Fernando Bello Imperial College London, Daniel Wheeler Guy's, King's and St Thomas' School of Medical Education, Kings College London, UK

Background/Purpose: Digital rectal examination (DRE) is an inherently difficult examination to teach due to its unsighted, tactile, and intimate nature. Tutors often have difficultly explaining and providing specific feedback for internal steps. Surveys of medical students reveal a lack of confidence and training in performing DRE. We propose and attempt to validate the use of novel simulation-based tools for teaching DRE.

Summary of the Innovation: 20 medical students were randomly allocated into 3 study arms: Control, See-through (ST) and See-through with Anatomy (STwA). Control arm were taught how to perform DRE with traditional methods, via a traditional benchtop model. For ST and STwA arms, position and pressure sensors on the examining finger allowed temporal, spatial, and pressure mapping on benchtop models in addition to visualisation of subjects' dynamic realtime performance on a computer simulated 'see- through' model. For STwA arm additional overlayed surrounding anatomy was illustrated. Qualitative evaluation of DRE learning experience was measured.

Conclusion: There were statistically significant differences between arms on feedback regarding learning $[F(2,17)=11.64, p=0.001]$. Post hoc test indicated statistically significant higher mean 5-point Likert scores for both ST $(4.29 \pm 0.49)$ and STwA (4.48 \pm 0.26$)$ arms, compared to control (3.22 \pm 0.69$)$. $100 \%$ of ST and STwA arm agreed that 'seeing through' helped them understand DRE better than the standard benchtop model. We propose that these high-fidelity See-through tools are impactful vehicles for the dissemination of modern DRE education for medical students and healthcare professionals internationally, and these concepts should be explored for further application in alternative intimate examinations.

DP 14-3 Multidisciplinary Healthcare and First Aid Provider Training for In-Flight Medical Emergencies: A Crowdsourcing Session followed by an Airplane Simulation

Anthony Seto University of Calgary, Josh Kariath University of Calgary

Background/Purpose: Is there a healthcare provider on board? Healthcare providers may be less confident for in-flight medical emergencies, as these situations are not part of their training curriculum or usual practice contexts (e.g. hospitals). A crowdsourcing session and simulation to mentally rehearse and practice clinical skills in airplane settings may improve their emergency preparedness.

Summary of the Innovation: This innovation involved: i) a session to crowdsource insights from multidisciplinary healthcare and first aid providers, followed by reviewing considerations of a CMAJ 2018 article on airplane emergencies, and ii) 2 airplane simulations (syncope and cardiac arrest). During crowdsourcing, 7 learning objectives of in-flight medical emergencies were explored: i) challenges, ii) solutions, iii) equipment, iv) taking vitals, v) general approach, vi) cardiac arrest approach, and vii) human resources / role-delegation. Knowledge and approaches extracted were then applied in simulations. Participants provided scores out of 7.00 
for: i) satisfaction of crowdsourcing session and simulation and ii) self-rated confidence on learning objectives at baseline, post-crowdsourcing session, and post-simulation. Results were analyzed with repeated measures ANOVA with post-hoc Tukey. Participants rated the crowdsourcing activity (6.70/7.00, $n=11)$ and simulation $(6.50 / 7.00, n=11)$ positively. Self-rated confidence in the 7 topics improved from baseline $(2.49 / 7.00)$ to postcrowdsourcing $(5.23 / 7.00)$ to post-simulation $(5.94 / 7.00)$, with significant differences $(p<0.01)$ between baseline and post-crowdsourcing, and between baseline and post-simulation. There was no significant difference between post-crowdsourcing and post-simulation.

Conclusion: Taken together, self-confidence in topics of in-flight medical emergencies may improve after just one crowdsourcing session, facilitated through group discussions and mental rehearsal. Added simulations may maintain self-confidence on these topics, by promoting memory retention through active learning and repetition.

\section{DP 14-4 Novel-Case Learning Environment for Surgical Education}

Regan Brownbridge University of Saskatchewan, Amit Persad University of Saskatchewan, Julia Radic University of Saskatchewan

Background/Purpose: Technological innovations have facilitated the introduction of case-based learning through virtual patient (VP) simulators into medical education. Current VP simulators usually consist of linear patient cases and multiple-choice questions, which limits the fidelity and educational potential of these simulations. We attempted to address these limitations by integrating natural language interaction and building branching cases with ample opportunity for the trainees to explore various outcomes related to their clinical decisions. Our learning tool provides trainees with a safe pseudo-clinical opportunity to perform in a leadership role. This type of innovation is sparsely mentioned in the literature. As so, our VP simulator will present a novel learning tool for neurosurgical trainees.
Summary of the Innovation: Two multifaceted neurosurgical cases (traumatic subdural hematoma and paediatric cerebral neoplasm) were created, reviewed, and integrated into a case exploration platform. Cases were chosen for their complexity, allowing for multiple key decision points. Each case entails trainees entering real-text commands to gather pertinent information, perform physical exams, order tests, interpret results, and make treatment decisions. Cases have multiple patient outcomes dependent on the trainee's clinical decision making. Educational documents at the end of each case serve to address any trainee knowledge deficits or reasoning errors.

Conclusion: Our case-based VP simulator provides neurosurgical trainees with a safe and structured oral exam style opportunity to practice and improve upon their clinical approach to standard neurosurgical patient presentations. This is a novel modality, not currently available or previously published in neurosurgical literature, that could be expanded for use in other fields.

\section{DP 14-5 Simulating the design and management of a medical clinic}

Astrid Bicamumpaka-Shema Université de Montréal, Sarah Zahabi McGill, Sarah Zahabi McGill, MarieFrédéric Tremblay Université de Montréal, Rosario Rodriguez McGill, Laura Rojas Rozo McGill

Background/Purpose: Under the present conditions of the Quebec healthcare system, the majority of physicians will practice in a community clinic. Furthermore, according to Pearl \& Fogel, "graduating students still lack the fundamental business and leadership training needed to [...] maximize quality and reduce cost in clinical practice," (NEJM, 2016). Considering this, we created a simulation activity for medical students and residents to learn the key stages in the drafting of a business plan for a medical clinic. This is the first simulation that aims at teaching an understanding of the complex nature of establishing a community clinic in Quebec. During the 2019 edition, we used a focus group and validated questionnaire to evaluate whether participation may have an impact on the understanding of administrative aspects of medical careers, on student and resident attitudes towards clinic development, 
and ultimately inspiring strong physician leadership with the goal of maximizing quality of care.

Methods: Participants were provided a guidebook with specific instructions and theoretical notions pertaining to each stage of clinic development (from mission and vision to budgeting). The collected data were integrated into the simulation software to assess many parameters including the number of patients seen in their clinic in a year, the type of appointment, and the clinic's expense/revenue ratio. Throughout the simulation, participants had access to professionals to guide their decision-making. Physicians, lawyers, tax experts, notaries and actuaries were assigned to mentor each team. Participants then prepared a five-minute presentation which was evaluated based on the following criteria: creativity, leadership, management skills and efficiency. At the closing of the activity, participants were invited to fill out a questionnaire and participate in a focus group.

Results: Out of the 72 participants of the third edition of the MCSC, 55 students responded to the survey (76.4\%). Overwhelmingly, respondents perceived (70.9\%) healthcare management and administration knowledge as useful for future doctors. However, only $56,4 \%$ of respondents find it essential for medical students to learn how to work in and operate a clinic. The MCSC helped 52 medical students $(94,5 \%)$ to develop an interest in improving healthcare delivery.

Conclusion: Medical student interest in healthcare administration as well as skill-building can be accomplished through the simulation of the creation of a medical clinic. Future studies can evaluate if the skills acquired are transferable to practice and lead to an increase in physician involvement in healthcare management and the implications of this on quality of care.

\section{DP 14-6 Speed accuracy tradeoffs in learning to tie surgical knots}

Luka Pusic Upper Canada College, Martin Pusic Harvard University, Mark Baxter Upper Canada College

Background/Purpose: We seek to understand if instructions to perform a task faster ("chronometric pressure") establish the conditions of the speedaccuracy trade-off.

Methods: Study design: This is a prospective cohort study of novices performing two-handed knot-tying for simulated vessel ligature. We compare each individual's performance upon verbal instructions to provoke varying chronometric pressure: "no pressure," "speed emphasis," "accuracy emphasis," "maximum speed." We determined their speed (timed in seconds), integrity of knots (yes/no) and the ratio of the two (=accuracy*100/time). Fitts' Law would predict: for no pressure, a high and variable accuracy ratio; for the speed and accuracy emphasis a consistent but lower ratio; and a yet lower ratio for the maximum speed condition. STUDY PROCEDURE: In a 3 hour session, after instruction and four warmup repetitions, 7 participants did 6 ligatures (36 throws) in each of the four conditions using 2-O Prolene. A SawBones knot tester with 10 pounds of pressure determined if knots held.

Results: Time per ligature differed across conditions $(p<0.007)$ as did the accuracy rate $(p<0.009)$. Speedaccuracy ratio stayed consistent and in the pattern predicted by Fitts' Law. Condition N \%Acc SD AvgTime(s) SD Ratio MaxSpeed 420.310 .4729 .57 12.921 .05 No Pressure 420.570 .5035 .2613 .531 .62 Accuracy 420.520 .5134 .6411 .011 .51 Speed 420.45 0.5029 .2115 .581 .55

Conclusion: We have shown that, using an authentic surgical task, the Fitts' Law conceptualization holds for novice learners under varying degrees of chronometric pressure. This instructional framework makes it possible optimize the degree of challenge and to provide quantitative feedback in situations where speed is balanced with accuracy (e.g. Csection; tracheostomy). 
DP 14-7 Use of a phlebotomy serious game for reducing pre-analytical errors made by blood-drawing health care practitioners

Adam Dubrowski Ontario Tech University (formerly UOIT), Tim Weber Ontario Tech University (formerly UOIT), Krystina Clarke Ontario Tech University (formerly UOIT), Zhujiang Wang Ontario Tech University (formerly UOIT), Robert Savaglio Ontario Tech University (formerly UOIT), Ruth Simpson Ontario Tech University (formerly UOIT), Maurice DiGiuseppe Ontario Tech University (formerly UOIT), Bill Kapralos Ontario Tech University (formerly UOIT)

Background/Purpose: Medical lab technologists (MLT) generally collect blood samples, however, in the absence of these human resources, other health care practitioners (HCPs) have to perform these skills. This is known as phlebotomy. Because of infrequent sample collection, HCPs may be less efficient and subject to pre-analytical errors. Since it is a procedure that falls within the scope of multiple health professions, it becomes prudent to develop and validate versatile, experiential training programs. We have developed a phlebotomy serious game (PSG) designed to train HCP's in the venipuncture process from supply and patient preparation through postcollection documentation.

Summary of the Innovation: The current version of the PSG consists of VR modules, hands-on physical models, and XR modules. We compared the PSG's learning objectives against the expected competencies of an entry-level MLT (CSMLS, 2019). Of the 95 expected sub-competencies, the alpha version of the PSG covers 14, and the current beta version can cover up to 93 .

Results: Current learning objectives within the PSG successful map onto $98 \%$ of the sub-competencies as articulated by CSMLS. Additionally, PSG objectives can also be mapped onto the CanMEDS key competencies in the roles of: Communicator, Leader, Health-Advocate, Scholar, Professional, and Medical Expert. Future directions include measuring face, content, and construct validity of the PSG, and longitudinal tests of educational efficacy and effectiveness. We expect the PSG will increase competency in both training and clinical settings, while reducing errors amongst MLTs and other HCPs.
DP 15-1 Sending a Shock through Electroconvulsive Therapy (ECT) Medical Education: Comparing Didactic Seminars-only versus Didactic Seminars Concomitant with High-Fidelity Simulation (HPS) in an Interprofessional Setting Amongst Psychiatry and Anesthesia Trainees

Sarah Frangou University of Saskatchewan, Kristen Edwards University of Saskatchewan, Natalie Garrett University of Saskatchewan, Joshua Frost University of Saskatchewan, Megan Deck University of Saskatchewan, Kiran Rabheru University of Ottawa, Peter Hedlin University of Saskatchewan, Marla Davidson University of Saskatchewan

Background/Purpose: Despite existing guidelines on ECT curriculum for medical residents, ECT training in Canada is not standardized. HPS-based training compared to traditional training has been shown to be superior in improving clinical skills. Thus, this study evaluated if HPS-based teaching will excel in improving knowledge, skills, confidence and interprofessional collaboration in delivering ECT in an interprofessional (IP) setting compared to traditional setting.

Methods: Psychiatry/Anesthesia residents' $(n=36)$ performance in ECT were evaluated in a double-blind randomized control trial. Testing on knowledge, skills, confidence and IP perceptions and collaboration was done utilizing: multiple choice questions, ECT Skills and Knowledge Confidence scale/modified Objective Structured Assessment of Technical Skills (OSATS)checklist and global rating scale and interdisciplinary education perception scale (IEP)/IP Collaborator Assessment Rubric, respectively. A series of pairedand independent sample t-tests examined the efficacy of HPS-based training.

Results: Participants, regardless of intervention type, gained knowledge, confidence and skills after training. Regardless of profession, HPS-exposed groups showed increased level of care of patients during ECT, skin/scalp preparation (OSATs subdomains) and total IEP scores $(p<0.05)$ compared to controls. Furthermore, the residents from both professions exposed to HPS showed improved skills in assessing response to ECT and understanding other profession's values relative to control. 
DP 15-2 Une activité de simulation de table à des fins de formation interprofessionnelle pour enseigner une nouvelle procédure intra-hospitalière de code rose: une étude pilote exploratoire et rétrospective sur la perception des apprenants

Amélie Frégeau Université de Montréal, Amélie Frégeau Université de Montréal, Alexis Cournoyer Université de Montréal, Nathalie Soucy Université de Montréal, David Fortier Université de Montréal, Pierre Desaulniers Université de Montréal, Véronique Castonguay Université de Montréal, Richard Fleet Université Laval

Background/Purpose: La formation interprofessionnnelle (FIP) vise à développer la collaboration interprofessionnelle (CIP) chez les travailleurs de la santé incluant la clarification des rôles. Par contre, le design pédagogique optimal des activités de FIP reste à définir. L'une des initiatives pédagogiques proposées est la simulation de table (TTX). Ainsi, nous avons tenté une FIP innovante avec une TTX ayant pour but d'enseigner un nouveau protocole de code rose intra-hospitalier dans les zones autres que le centre des naissances, la néonatalogie et le bloc opératoire. L'objectif primaire de l'étude est d'évaluer la perception des participants à propos de cette FIP. >

Methods: Cette étude est rétrospective. Un groupe interprofessionnel a été recruté. Immédiatement après la $T T X$, un sondage de satisfaction a été remis aux participants. Des statistiques descriptives ( $n, \%)$ ont été effectuées. Les commentaires recueillis lors du débreffage ont permis de nuancer les résultats.

Results: Treize professionnels ont participé à la TTX. De ces 13 professionnels, 10 ont répondus au sondage $(10 / 13: 77 \%)$. Suite à la TTX, $80 \%(n=8)$ des participants ont eu l'impression de mieux comprendre leur propre rôle et $90 \% \quad(n=9)$ des participants ont eu l'impression de mieux comprendre le rôle des autres professionnels. Tous ont apprécié la TTX et tous ont affirmés qu'il était probable ou très probable qu'ils participent à nouveau à une telle activité s'ils y étaient invités ou qu'ils recommanderaient à un collègue d'y participer.
Conclusion: La TTX a été appréciée par les participants et les apprenants se sentent globalement plus confiants dans leur rôle et dans leur connaissance du rôle des autres professionnels. Le bénéfice de la TTX pour la FIP par rapport à d'autres modalités de simulation reste encore à prouver.

\section{DP 15-3 Evaluating the integration of interprofessional collaboration competencies within simulation training}

Minisha Suri Canadian Memorial Chiropractic College / St. Michael's Hospital, Deborah Kopansky-Giles University of Toronto, Silvano Mior Canadian Memorial Chiropractic College, Lianne Jeffs University of Toronto, Kari White St. Michael's Hospital, Douglas Campbell St. Michael's Hospital

Background/Purpose: At St. Michael's Hospital, an interprofessional collaboration competency framework (IPCCF) was developed to improve collaborative competency and support team-based care. This study evaluated whether formally embedding domains from the IPCCF within team simulation-based medical education (SBME) enhanced practitioner competency compared to usual simulation education.

Methods: A randomized control trial with concurrent mixed methods was used. Participants were randomized to receive either pre-simulation education (Intervention) or usual SBME pre-briefing (Control). Self-perceived collaborative competency was measured using the Health Professional Collaborative Competency Perception Scale (HPCCPS). Qualitative thematic analysis of debriefing audio-video data was used to explore participant perception of IPC.

Results: Intervention and control groups were similar demographically (I: $n=28 ; C: n=25)$. There were no significant differences in mean change on HPCCPS between groups. In debriefing, intervention groups generated increased dialogue around communication, teamwork and role awareness, and initiated more self-reflection on IPC than control participants. Facilitators frequently adopted a teaching role, which may have inhibited dialogue surrounding IPC. 
Conclusion: Results suggest that pre-simulation education on IPC competencies did not affect participants' self-reported collaboration. It did lead to greater discourse about IPC, which may result positively in attitudes toward and ability to collaborate. Results may inform future delivery and assessment of SBME intended to enhance IPC competencies.

\section{DP 15-4 Implementing the 2017 Canadian} Opioid Prescribing Guideline within the Thamesview Family Health Team

Jackson Blonde Western University, Andrew Atkins Thamesview Family Health Team, Susan Munro Western University

Background/Purpose: Canadians are in the midst of an opioid crisis; a crisis driven in part by prescription opioids. Canada has the second highest rate of opioid prescribing per capita in the world. Within Ontario, the Erie St. Clair Local Health Integration Network (LHIN) is among the highest of 14 in terms of opioid prescribing rates. Given that the Thamesview Family Health Team (TFHT) is within this LHIN, as well as the risks and harms associated with prescription opioids, the present study aimed to evaluate the opioid prescribing practices of the TFHT.

Methods: Opioid prescribing practices were evaluated based on the 2017 Canadian Opioid Prescribing Guideline. Using Practice Solutions Suite, we identified all patients within the TFHT who had an active opioid prescription from June 2018-June 2019. We then used the Opioid Toolbar to calculate the morphine equivalent (MEQ) for each patient. Lastly, we interviewed 17 physicians regarding their patients with an MEQ $>=90$, their approach to managing patients with chronic non-cancer pain (CNCP), and their attitudes toward the guideline.

Results: Five percent of rostered patients had an active opioid prescription. Of these patients, $10 \%$ had an MEQ $>=90$. Physician's approaches to managing patients with CNCP and those with an MEQ $>=90$ were multimodal. Most physicians supported the guideline but found it unrealistic in certain situations. Six physicians began implementing the Opioid Toolbar.
Conclusion: Physicians of the TFHT are implementing the 2017 guideline into their practices. Furthermore, the Opioid Toolbar provided an effective way of evaluating opioid prescribing practices.

DP 15-5 Evaluating the impact of studentled interprofessional musculoskeletal (MSK) workshops on knowledge and confidence of medical students regarding the MSK assessment.

Cyril Boulila McGill, Élise Girouard-Chantal McGill, Matthew Lassman McGill, Christophe Gendron McGill, Timothy Dubé Université de Sherbrooke, Elise Girourard-Chantal McGill

Background/Purpose: The system medical (MD) students feel the least confident about when graduating is the musculoskeletal (MSK) system. In comparison, physiotherapy (PT) programs dedicate an extensive amount of time on the MSK exam. This premise led a group of medical students to create a series of interprofessional (IP) MSK hands-on workshops where PT students collaborate with MD students to review anatomy and practice the MSK exam. The objective of this study is to determine whether participation in peer-learning IP workshops increases knowledge and confidence of MD students regarding MSK exam.

Methods: McGill University MD students ( $N=9$ ) attended 3 workshops on a voluntary basis, each covering a different joint. Pre-post workshop knowledge quizzes and confidence questionnaires were used to assess short-term (immediate) and longterm ( 6 months) change in knowledge and confidence level related to the MSK exam. A focus group interview $(\mathrm{N}=4)$ was conducted to gain an in-depth understanding of MD students' perspectives about the workshops.

Results: Results show a significant increase in knowledge quiz scores post-workshops (mean: +2.4 , $p=0,013$ ) with immediate increase of $25 \%$ and longterm increase of $13 \%$. At baseline, $7 / 9$ (78\%) participants identified as not confident in performing an MSK exam, 0 participant identified as such postworkshops. Qualitative reports show MD students highly value this learning environment, find hands-on learning activities lacking in MD curricula and IP 
learning allows for realistic contexts which reinforces knowledge acquisition.

Conclusion: Student-led IP MSK workshops have been effective in advancing knowledge and increasing confidence regarding assessment of the MSK system, representing a promising avenue for medical education curricula.

\section{DP 15-6 The Patient and Family Narratives Seminars at the University of Saskatchewan}

Marcel D'Eon University of Saskatchewan, Get Lombamo University of Saskatchewan, Jelyssa Luc University of Saskatchewan, Natasha Hubbard Murdoch Saskatchewan Polytechnic, Poppy Lowe University of Saskatchewan

Background/Purpose: The patient's experience of their illness is an important consideration in providing effective and compassionate health care. The Patient and Family Narratives (PFN) seminars at the University of Saskatchewan were created to provide a venue for health professions students to experience stories of patient journeys through illness and treatment. Since 2010, these 90-minute PFN sessions have provided up to 500 student experiences each year. During each PFN session, a patient, often accompanied by a family member, takes 30-40 minutes to share parts of their personal illness story and select encounters with health care providers. In small interprofessional groups, students discuss relevant questions.

Methods: We analyzed hundreds of student comments collected over the last few years. We then developed a survey and sent it to the 196 PFN student attendees in 2018-2019. The survey asked participants to rate features of the PFN utilizing a 6point scale from "strongly dissatisfied" to "strongly satisfied". The Behavioral Research Ethics Board of the University of Saskatchewan granted an exemption from ethics review since this study was designated a program evaluation.

Results: Fifty students (response rate of $25.1 \%$ ) completed our survey representing over 150 individual student experiences. Students rated highly the PFN seminars overall and the patient stories alone: means of 4.3 and 4.9 respectively. Students rated the small group discussions at 3.3.
Conclusion: We believe that the PFN seminars have contributed to the education of health professions students by connecting them with patients and their stories. We will offer PFN sessions in 2019-2020 but will improve the structure of the small interprofessional groups.

DP 15-7 Multi-professional catalyst to the design, research and implementation of state-of-the-art simulation in health professions education

Krystina Clarke Ontario Tech University, Timothy Weber Ontario Tech University, Zhujiang Wang Ontario Tech University, Adam Dubrowski Ontario Tech University, Alvaro Quevedo Ontario Tech University, Bill Kapralos Ontario Tech University

Background/Purpose: Simulation is the virtual, physical, or hybrid replication of a task/event for the purpose of training and assessment and must be contextually accurate to be functional. Therefore, a single profession does not suffice when designing simulation experiences, but instead several professions must work together. To address this, we have developed maxSIMhealth (MSH).

Summary of the Innovation: $\mathrm{MSH}$ is a multidisciplinary collaborative of manufacturing, design, and simulation labs at Ontario Tech University combining expertise in Health Sciences, Business and $\mathrm{IT}$, Engineering, and more while building community partnerships to advance simulation training. MHS' vision is to advance knowledge discovery and application that revolutionizes health by offering innovative solutions for simulation training and clinical application. It discovers existing simulation gaps, provides innovative solutions that change systems, and leads to improved healthcare outcomes. Specifically, we utilize disruptive technologies (3D printing, gaming, extended reality) as innovative solutions that deliver cost-effective, portable, and realistic simulation, which is currently lacking. Our work spans a broad spectrum of scholarship from mapping existing gaps, to changing education systems, to improving learning and performance outcomes. To ensure timely and meaningful knowledge translation, we have established an institutional channel within the Cureus Journal for 
Medical Sciences where our work is freely disseminated as peer-reviewed, PubMed-indexed publications.

Conclusion: $\mathrm{MSH}$ is a novel collaborative innovation with aims to develop future cohorts of scholars with strong competencies ranging from technology application, to collaborating in new environments, to communicating professionally and problem-solving. Our work will transform the current health professional education landscape by providing novel, flexible, and inexpensive simulation experiences.

\section{DP 15-8 Introduction to Biosecurity and} Bioterrorism: An Educational Curriculum Response to a Continued Knowledge Threat

Chris Winstead-Derlega Stanford University, Stefanie Sebok-Syer Stanford University, Michelle Bach Stanford University, Elizabeth Trinh Stanford University, Natalia Trounce, Milana Boukhman Stanford University

Background/Purpose: Biological threats, such as B. anthracis in 2001, SARS in 2003, H1N1 in 2009, Ebola in 2014, and Zika in 2016, challenge international health security. In 2003, the Association of American Medical Colleges (AAMC) recommended incorporating a biosecurity curriculum into undergraduate medical education. While previous curricula focused on structured responses, we introduce an established curriculum that includes didactics and problem-based learning to teach students innovative solutions to biothreats and bioterrorism.

Summary of the Innovation: First introduced in 2010, Introduction to Biosecurity and Bioterrorism is a tenweek course taught biweekly within the School of Medicine at Stanford University. Designed to reflect the dynamic and interdisciplinary field of biosecurity, the course constructs a foundation of knowledge with face-to-face didactics from national biosecurity experts. The co-construction of knowledge from students across medicine, engineering and technology development, public health and policy, allow for an interprofessional approach and appreciation of biosecurity threats. Our instructional methods include: core readings, active engagement with experts during didactic lectures, participation in group discussions, and completion of a small group innovation solutions project.

Conclusion: Medical schools have struggled to incorporate biosecurity topics into traditional models of undergraduate medical education. This curriculum highlights an established biosecurity curriculum that integrates didactics and problem-based learning to prepare students for future roles in biosecurity.

DP 15-9 Developing a On-Call Duty Toolkit for Junior Residents at a Cardiology Intensive Care Unit: An Interdisciplinary Collaboration between Nurses and Medical Residents

Camille Pelletier Vernooy Université de Montréal, Clément Cohé Centre hospitalier universitaire de Montréal (CHUM)

Background/Purpose: On-call duty at Cardiology Intensive Care Units (CICU) is challenging for junior residents especially during night shifts, as they manage ill patients, while advice from senior residents is only available via telephone. Nurses with many years of experience play a substantial role in teaching junior residents medical procedures. A review of the literature reports that nursing contributions to medical education during on-call duty are poorly acknowledged and represent an untapped potential.

Summary of the Innovation: The CICU at the Centre hospitalier universitaire de Montréal is a non-surgical twenty beds department staffed by junior residents during night shifts. To date, no formal documentation has been available to support them. A twenty page toolkit was created by second year residents in collaboration with nurses cumulating each more than ten years of experience. The aim was to assess expectations of both junior residents and nurses. The toolkit is a guide for the management of common cardiac pathologies and complications and is not meant to replace official guidelines. One chapter aspires to fulfil gaps in knowledge about hospital protocols and clinical procedures identified by nurses. The toolkit was approved by the CICU Medical Director and is now used by forty junior residents. Satisfaction assessment shows that new junior residents felt more confident after reading it. 
Conclusion: In the era of multidisciplinary health care teams, sharing knowledge across disciplines is of cardinal importance. The toolkit formally recognised the necessary collaboration between on-call duty medical residents and nurses and provides a tangible support for new junior residents.

\section{DP 16-1 Queerying the Foundations: an} evaluation of the LGBTQ health curriculum in undergraduate medical education at the University of Toronto

Kira Abelsohn University of Toronto, James Owen University of Toronto

Background/Purpose: There is growing evidence demonstrating that increased exposure to LGBTQ health content in medical school curriculum increases student knowledge about LGBTQ health, improves the performance of medical learners in the delivery of health care services to LGBTQ patients, reduces stigma from medical learners towards LGBTQ patients, and increases medical learners' willingness to treat LGBTQ patients in their future practice (Kelly et al, 2008; Sanchez, et al, 2006; Strong, 2013). In 2015, the University of Toronto developed and launched a new integrated Foundations Curriculum on LGBTQ health. The purpose of this study was to evaluate this new curriculum and assess its' impact on student's knowledge of LGBTQ health and comfort level working with LGBTQ populations.

Methods: First year medical students who had not yet received the LGBTQ Foundations curriculum and second year medical students who had completed the curriculum were asked to complete an anonymous and confidential survey evaluation via email. This survey included the Lesbian, Gay, Bisexual, and Transgender Development of Clinical Skills Scale (LGBT-DOCSS), a newly validated 15-item-scale designed for interdisciplinary clinical assessment of health providers (Bidell, 2017).

Results: A total of 73 medical students completed the survey. The LGBT-DOCSS scale is analyzed as a total scale, as well as three independent sub-scales: knowledge, clinical preparedness and attitudes. All scores are calculated out of 7. The LGBT-DOCSS total score among first year medical students was 4.9 , vs 5.7 in second year students, a difference of $11.4 \%$ $(p<0.0001$. Our analysis demonstrates an increase in LGBTQ-DOCSS total score, as well as all sub-scores including LGBTQ clinical preparedness, LGBTQ health knowledge and attitudes towards LGBTQ population, in the group receiving the new curriculum.

Conclusion: To our knowledge, University of Toronto undergraduate medical curriculum is the first in Canada to fully integrate LGBTQ objectives into undergraduate medical education. The evaluation data will be used to continuously improve our curriculum to meet the evolving needs of undergraduate learners, and contribute to building a shared understanding of LGBTQ curricular development across Canadian medical schools.

\section{DP 16-2 Decolonizing Evaluation: A Case Study}

Cathy Fournier Wilson Centre, David Rojas University of Toronto, Lisa Richardson University of Toronto, Cynthia Whitehead Wilson Centre, Sadie King Women's College Hospital

Background/Purpose: In response to the Truth and Reconciliation Commission Report and its Calls to Action, Women's College Hospital (WCH) is currently working towards developing and implementing strategies to help transform the institution to become a safe and more inclusive space for Indigenous Peoples. One key step towards this goal is the development of an Indigenized evaluation model/framework to better understand institutional transformation to ensure that the initiatives/programs implemented best meet the needs of Indigenous Peoples.

Summary of the Innovation: In an effort to address the limits of using Western forms of evaluation we are engaging in a process of working to decolonize the evaluation practices of indigenous services at $\mathrm{WCH}$. To do so, we are using the 'Wise Practices" guidelines to ensure that Indigenous knowledges and perspectives are acknowledged, captured and included when evaluating the institutional transformation that WCH is going through to create safer and more inclusive health care for Indigenous Peoples. Decolonizing evaluation is a complex, multilayered and ongoing process. The Wise Practice approach highlights that there is no one size fits all 
and also challenges the 'best' practices model by commanding a critical awareness of who and how it is decided which practices are best as well and who they are best for.

Conclusion: Working towards decolonizing evaluation requires a critical look at Western colonial forms of evaluation and evaluative markers while simultaneously trying to decolonize one's own perspective and which knowledges/perspectives are valued over others

\section{DP 16-3 Citizen Forums on Access to} Indigenous Health Education: An Innovative Initiative of the Faculté de médecine de l'Université de Montréal

Ahmed Maherzi Université de Montréal, Samuel Blain Université de Montréal, Claudie Paul Regroupement des centres d'amitié autochtones du Québec, Laurianne Petiquay Centre d'amitié autochtone de La Tuque, Claudia Petiquay Centre d'amitié autochtone de Trois-Rivières

Background/Purpose: The Association of Faculties of Medicine of Canada (AFMC) has a long-term commitment to promoting social accountability (SA) and indigenous health. The Faculté de médecine de I'Université de Montréal intends to contribute to this commitment by implementing a major partnership project that targets and involves the indigenous populations it is mandated to serve.

Summary of the Innovation: The project is the result of a collaborative partnership between the Faculty's SA office and the Regroupement des centres d'amitié autochtones du Québec (RCAAQ): it aims to better understand the specific health training needs of indigenous populations, particularly in identifying issues related to its accessibility and relevance, coconstructing a concerted action plan and monitoring its implementation. Our second goal is to educate our students and our colleagues teaching in health sciences about Indigenous realities and their culture. The priority populations targeted in this project are urban Indigenous communities of La Tuque and TroisRivières. Three citizen forums were organized between March and May 2019: Two local forums and a regional forum attended by 62 participants including 40 indigenous made it possible to identify issues and courses of action. Five areas of work have been identified to structure our action plan.

Conclusion: This public consultation process has established a relationship of trust with the Indigenous community and co-constructed this academic project together. This approach will serve as a model for future planned projects with other vulnerable populations served by our faculty.

DP 16-4 What makes medical students care? Predictors of interest in Indigenous health learning and clinical practice

Sharon Yeung Queen's University, Amy Bombay Dalhousie University, Chad Walker Queen's University, Jeff Denis McMaster University, Debbie Martin Dalhousie University, Paul Sylvestre Queen's University, Heather Castleden Queen's University

Background/Purpose: Including curricula on Indigenous health in medical school is an acknowledged prerequisite for reducing Indigenous health disparities in Canada. However, little is known about medical students' knowledge and interest in Indigenous health at entry to medical school, and it is unclear whether current Indigenous health curricula successfully impacts their beliefs and behaviours.

Methods: Students $(n=129)$ at one Canadian medical school completed a survey that evaluated their sociopolitical attitudes towards Indigenous people, knowledge of colonization and its links to Indigenous health inequities, knowledge of Indigenous health inequities, and self-rated educational preparedness to work with Indigenous patients. Students' perceived importance of learning about Indigenous peoples, and interest in working in an Indigenous community, were assessed as outcomes. Survey items were grouped into five independent factors using principal component analysis and outcomes were modelled using multivariate regression analysis.

Results: Students reported strong interest in Indigenous health but did not believe themselves adequately educated or prepared to work in Indigenous communities. When controlling for age and gender, a significant predictor of both (1) perceived importance of learning about Indigenous health and (2) interest in working in an Indigenous community was positive sociopolitical attitudes about 
Indigenous peoples. Knowledge about Indigenous health inequities was negatively associated with interest in working in an Indigenous community.

Conclusion: In addition to teaching knowledge-based concepts, medical educators must consider the importance of attitude change in designing Indigenous health curricula to shape students' behaviours and ultimately improve physician relationships with Indigenous patients.

\section{DP 16-5 Applying Appreciative Inquiry to Promote Medical Student Diversity}

Ashton Cox University of Alberta, Hollis Lai University of Alberta, Tracey Hillier University of Alberta, Joanne Rodger University of Alberta, Thomas Jeffery University of Alberta

Background/Purpose: With an increasing focus on attracting diverse students into a career in medicine, more outreach approaches are needed to showcase the different paths and lived experiences of students admitted to medical school. The purpose of this study is to explore how appreciative inquiry, a popular approach in change management, can be applied to promote student diversity in medical education.

Summary of the Innovation: This study is conducted in three phases. First, an interview protocol was developed through an iterative process from two interviewees. A set of 15 questions from three domains (path to medicine, reflection, and emotions on medicine) was devised to provide a semistructured interview process in exploring how students recall and reflect on their positive experiences in medical school. Second, a pilot of ten participants were interviewed in 30 minute in-person sessions. Participants included recent graduates and current students. Third, a collection of experience vignettes from each participant was then produced to share the successes, experiences, and challenges each student faced in entering medicine.

Conclusion: The vignettes were storified and shared to promote and encourage students with a diverse set of experiences, training, and skills to consider a career in medicine. Appreciative inquiry is an informative method in collecting powerful memories and experiences in narrative form. Future study may expand on this method to validate and enable a broader participation of students across the program to further demonstrate and celebrate the diversity of students admitted to our medical education program.

DP 16-6 Transgender Health in Emergency Medicine: A Needs Assessment for Development of Online Curricula

Devon Stride McMaster University, Teresa Chan McMaster University

Background/Purpose: Transgender (trans-) people face significant barriers to accessing emergency healthcare, including delay or refusal of care, and harassment. A contributor is a lack of education in medicine curricula dedicated to gender and health. The purpose of this needs assessment was to identify current gaps in trans-health knowledge in emergency medicine.

Methods: We conducted a mixed methods, multiphase needs assessment, with the intention of discovering topics relevant to clinical clerks in emergency medicine. Data was collected from medical students attending the Canadian Association of Emergency Physicians Conference. Students participated in a written survey and/or focus group. Data was hand coded by the primary researcher and checked by a second collaborator. A simple thematic analysis was reported and used as the basis of curriculum design. We received an ethics exemption from our institutional review board.

Results: In total, 44 students participated in the survey $(n=44)$ and focus group $(n=12)$. Respondents represented nine Canadian medical schools. LGBTQ+ health teaching ranged from none to three sessions. Approximately half of respondents reported poor knowledge of trans-related terms. Other themes included lack of approach in sensitive histories or physical exams, and perceived inability to advocate for trans-patients with staff physicians.

Conclusion: The results of this needs assessment demonstrated a significant gap of trans-healthcare knowledge. Respondents identified key gaps, including gender-related terms, sensitive encounters, and advocating for trans-patients. The results of this needs assessments are pertinent, as they will guide the development of modules for a Canadian EM website (CanadiEM.org). 
DP 16-7 Addressing the need for ethnically diverse stem cell donors in registries globally

Valerie Hladky Université de Montréal, Amelia Lamontagne Université de Montréal, Valerie Hladky Université de Montréal, Amelia Lamontagne Université de Montréal, Qi Li Université de Montréal

Background/Purpose: Physicians have a social responsibility to advocate for patients and ensure equity in healthcare for all. However, there exist inherent disparities amongst patients of different ethnicities in access to stem cell transplants. This is mostly attributed to the lack of non-Caucasian donors. As Canada's population grows more diverse, it is imperative to address this issue by educating young adults of the status quo, and ways in which it could be called into question.

Summary of the Innovation: As such, we implemented "Donnons la Chance au Don" (DCD). DCD is a 60-minute interactive workshop intended to inform, sensitize, and mobilize high school and CÉGEP students on the issue of a lack of racial/ethnic diversity in stem cell donor registries. In collaboration with IFMSA-Québec and the Swab the World Foundation, students are provided with a brief overview of the science behind stem cell transplants (including the challenges associated with genetic compatibility), along with an up-to-date account of the state of registries worldwide. We explore and suggests avenues in which students can become responsible citizens and better the lives of patients awaiting stem cell transplants.

Conclusion: Following this inaugural pilot year, DCD has been well received by students and teachers alike. 19 workshops were held in 4 anglophone and francophone Montreal schools by medical school students from Université de Montréal and Université Laval. A participant satisfaction survey measured outcomes related to the workshop. Out of approximately 700 students reached, $99 \%$ reported having a better understanding of stem cell donation, 95\% are more conscious of current inequities in access to transplants, and $65 \%$ are more likely join the stem cell donor registry at the age of 18 .
DP 16-8 Get Cultured: A Directory of Inclusive Supports for Black \& Indigenous Medical Students at Ontario Medical Schools

Chantal Phillips University of Toronto, Anita Balakrishna University of Toronto, Lisa Robinson University of Toronto

Background/Purpose: Cultural safety and inclusion have become relevant themes in Canadian undergraduate medical education to reduce unsafe medical practices affecting Black and Indigenous patients. Less is known about culturally safe and inclusion-based initiatives supporting the social and learning environments of matriculating medical students from these populations. Culturally unsafe environments have been shown to increase burnout and depression within student cohorts. In effect, this knowledge gap of available resources hinders Black and Indigenous medical students and administrations aiming to maintain representation of these students.

Methods: The objective of this study was to identify all staff, programs, and policies implemented to foster the cultural safety and inclusion of Black and Indigenous medical students. A grey literature search of the six Ontario medical school websites was conducted between July and August 2019. Relevant sources were organized according to leadership, organizational policies, student supports, and curriculum.

Results: Each school has established staff in the area of Indigenous affairs, with variations in leadership supporting diversity and Black students specifically. Diversity statements also differ in their specificity to Black and Indigenous students. Every school has a cultural competency curriculum, although two specifically refer to cultural safety. Student supports vary broadly, including scholarships specific to Black and Indigenous students, student-run initiatives, conferences, and mentorship programs.

Conclusion: By publicly mapping these initiatives, students are better connected to available resources and can optimize utilization. Administrations can also identify areas of strength and improvement, with the ultimate goal of graduating medical student bodies representative of the Canadian population. 
DP 17-1 Exploring the impact of the Surgical Exploration and Discovery (SEAD) Program on medical students' perceptions of gender biases in surgery: a mixed-method evaluation

Mimi Deng University of Ottawa, Christine Seabrook The Ottawa Hospital, Emily (Tu Nhi) Nham University of Ottawa, James Watterson The Ottawa Hospital, Anahita Malvea University of Ottawa

Background/Purpose: Female representation in surgery is increasing and several factors may contribute to this trend. The objective of the study is to assess the role of the Surgical Exploration and Discovery (SEAD), a 2-week immersive surgical summer program, on medical students' interest in surgery and gender-based perceptions of the field.

Methods: Students' gender biases in surgery were assessed using a modified version of the Gender-Bias in Medical Education Scale (GBMES) administered pre- and post-SEAD. Mean difference for each item pre- and post-SEAD was calculated and differences between SEAD ( $n=22 ; M / F=12 / 10)$ and non-SEAD ( $n=18 ; M / F=7 / 11)$ participants as well as between male and female SEAD participants were analyzed using t-test. Students' free-text responses were qualitatively assessed to further determine experiences and attitudes regarding gender bias in surgery.

Results: Levels of interest in surgery did not significantly change between groups $(P=0.325)$. SEAD participants had significant reductions in the strength of the following beliefs compared to controls: "Surgery is male-dominated", "Medical studies are mainly done in males", "Gender discrimination is more pronounce in surgery than other medical professions", and "Consideration of my gender is an important factor in whether or not to pursue surgery as a career" $(P<0.05)$. Stratified analysis revealed that the significance of these reductions were owing to female participants. Qualitative analysis revealed variable attitudes and experiences regarding gender biases in surgery.

Conclusion: Early surgical exposure through SEAD reduces gender bias in surgery, particularly in female medical students.
DP 17-2 Representation of family medicine in preclerkship clinical cases: Missed opportunities?

Francis Diaz University of Manitoba, Anita Ens University of Manitoba, Sasha Thiem University of Manitoba, Amanda Condon University of Manitoba,

Background/Purpose: The Canadian College of Family Physicians introduced the Patient's Medical Home model in 2011 with a subsequent update in 2019. This evolving model of family medicine emphasizes access, continuity, comprehensiveness and team-based care that is community-centred and responsive. While the Patient's Medical Home is becoming more common, we sought to explore the degree to which preclerkship small group and assigned study clinical cases incorporate these elements of clinical care delivery.

Methoids: All available clinical cases used in Year 1 and Year 2 preclerkship small group and assigned studies at the University of Manitoba were reviewed. Cases were analyzed for: specified care provider, patient age, type of clinical case, location, setting, referral source, resources, learners, technology, interprofessionalism, continuity and patientcentredness. Using stratified random sampling, 50 cases (6\%) were reviewed by four researchers to discuss classification and analysis. Agreement was reached through consensus.

Results: 844 cases were reviewed with 827 cases included in the final analysis. Of these, $47 \%$ of cases were from Year 1 and 53\% from Year 2. 13\% identified a family physician as a provider. $7 \%$ identified continuity and $8 \%$ identified patient-centredness. Only a few cases identified a referral source (6\%), other team members $(8 \%)$, community resources $(1 \%)$, learners $(5 \%)$ or technology $(2 \%)$. A large number of cases had an unspecified care provider $(78 \%)$, case setting $(45 \%)$ or case location (91\%).

Conclusion: The Patient's Medical Home model is minimally represented in preclerkship clinical cases. Excellent opportunities exist to represent modern care delivery and family medicine in preclerkship with minimal case reconstruction. 
DP 17-3 Assessment of medical student experiences and perceptions of practical surgical teaching throughout UK undergraduate medical education

Daniel Wheeler Guy's, King's and St Thomas' School of Medical Education, King's College London, UK, Filippos Papadopoulos Guy's, King's and St Thomas' School of Medical Education, King's College London, UK, Louise Hufton

Background/Purpose: UK medical student exposure to surgical teaching is broadly limited to didactic lecture-based content, passive theatre observation, or often a single suturing session. Given the inherent dexterity required of surgical techniques, we evaluated medical student perspectives and exposure to practical surgical teaching.

Methods: This was a cross-sectional, descriptive study for UK medical students. The study tool was a questionnaire comprising 5-point Likert scales and open-ended qualitative questions, reaching 230 students at 10 medical schools over a one-week enrolment. Qualitative data underwent thematic analysis to quantitively model past experiences as Integrated Curriculum (IC), Supplementary Workshops (SW), or Advanced Simulation Training (AST). We also compared metrics to pre-/post-course responses from an undergraduate microsurgical course that we recently developed.

Results: 119 respondents (51.7\% response rate), mean year of study $3.32 \pm 1.39$ (range 1-6), were categorised as $12.6 \%$ AST, $22.7 \%$ SW, and $64.7 \%$ IC. The mean response to if practical training would influence career aspirations was $4.13 \pm 0.92$, and to considering a career in surgery $3.38 \pm 1.31$, which was similar to pre-microsurgical course responses $3.94 \pm 0.93(p=0.1008)$ but had a statistically significant lower response versus post-course responses $4.63 \pm 0.62(p=0.0003)$. The response to curriculum integration of surgical practical skills was $1.75 \pm 1.01$, and to support implementation $4.38 \pm 0.78$.

Conclusion: Our findings suggest poor current integration of practical surgical skills teaching in the UK undergraduate medical curriculum. We outline a defined demand for future implementation and propose that gaining simulation exposure increases engagement in pursuing surgical careers, vital for future attraction and retention to surgical specialties.

DP 17-4 Addressing Barriers to Learning Geriatric Medicine in Preclerkship

Danielle Portnoy University of Alberta, Martin Moran University of Alberta, Tracey Hillier University of Alberta

Background/Purpose: Learners report both a lack of education and a lack of positive role models as barriers to learning to care for the elderly (Meiboom et al 2015). The elderly population is the fastest growing population group in Canada and most physicians- whatever their discipline- will be caring for this group and most care will be provided in the community. Learning is most effective when it incorporates self-testing and reinforcement (Dunlosky et al. 2013). However, most medical schools in Canada provide teaching through lectures (which traditionally do not incorporate self-testing or reinforcement) and do not provide a compulsory geriatrics clerkship; for those that do, it tends to occur late in the undergraduate curriculum (Parmar et al. 2009). The cumulative effect is limited education.

Summary of the Innovation: At the University of Alberta, a pre-clerkship elective has been developed targeting early exposure to clinical geriatrics in the community with effective learning strategies and leadership through seniors educating learners and a question and answer session with a medical leader in geriatrics. A 12 hour elective comprising an experiential visit to an award-winning seniors centre, clerking for a preceptor providing care in assisted living, case-based learning involving a skill, cognitive screening test, themed around the topical issue of driving assessments for seniors, and a Q\&A session with an inspiring medical leader.

Conclusion: On measures of attitude change, medical students viewed geriatrics more positively and intended to use a cognitive screening test in their clinical practice. 
DP 17-5 From Sea to Sea: Undergraduate Medical Education in Medical Genetics

Shaimaa Helal Queen's University, Andrea Guerin Queen's University, Hanna Faghfoury Queen's University

Background/Purpose: There is little data on genetics teaching in Canadian medical schools. The objectives of this study were to: 1 . Enumerate the hours of teaching 2. Describe the timing of delivery of content 3. Delineate the type of instruction 4. Describe the various professionals that teach 5 . Outline the factors that inform objectives

Methods: Undergraduate genetics leads were identified. A 10-question survey collecting data was sent via email to the leads at the 15 Canadian medical schools.

Results: Survey results were compiled and examined. The survey was completed by $11 / 15$ (73\%) of the leads. Most $(90 \%)$ of content was delivered in preclerkship with $70 \%$ of schools having at least 16 hours. Lecture was utilized by all of schools, followed by small group sessions (72\%). In one school, genetics was integrated into PBL tutorials. Independent learning events and patient contact sessions were also used to lesser degrees. Teaching teams varied. All schools had a MD Geneticist, with $90 \%$ using Genetic counselors, 63\% Laboratory Geneticists, 45.5\% physician trainees and $54.5 \%$ patients. Objectives for the curriculum were not uniform. Most were determined by either the course director/clinician $(60 \%)$ or the university $(40 \%)$ or combination.

Conclusion: Currently, there is no standardization for undergraduate medical education in Medical Genetics. Timing of teaching is mainly in preclerkship, with a variety of teaching modalities and varied team. Understanding the current landscape of Medical Genetics teaching is useful in standardizing teaching, objectives and sharing of resources among the leads at various schools.
DP 17-6 The effect of surgical observerships on the perceptions and career choices of preclinical medical students, a mixed methods study

Maureen Thivierge-Southidara Université de Montréal, Mathieu Courchesne Université de Montréal, Steven Bonneau Université de Montréal, Michel Carrier Université de Montréal, Margaret Henri Université de Montréal

Background/Purpose: Students are increasingly choosing non-surgical specialties. Observership programs can address factors influencing medical students towards surgical careers by allowing preclerkship exposure, mentorship, and changing misconceptions.

Methods: A quasi-experimental convergent mixed methods questionnaire study design was used to determine the influence of a peer-initiated observership program on the career choices of preclinical medical students, and the factors pertaining to a positive experience. A McNemar test was used to evaluate the impact on students' career choices. An inductive thematic analysis was used to analyze students' reasoning.

Results: Of 204 participating students, 85 (54.1\%) answered both questionnaires. Most were interested in a surgical specialty prior to $(84.7 \%, \mathrm{n}=72)$ and after (84.0\%, $n=68)$ the observership. There were no statistically significant differences according to change of interest $(p=1.00)$. However, most students $(80.9 \%, n=68)$ reported being more interested in a surgical career as a result of the observership which allowed them to determine that the type of practice they considered was congruent with a surgical career. Their perceptions of the surgical field became positive, particularly towards its pace, climate and the humanistic patient-doctor relationship it required. The experience was influenced by surgeons' and teams' attitudes towards students, knowledge sharing, and quality of exposure. Students mentioned that their own willingness to participate was crucial for a successful experience.

Results: This student-led observership program allowed an early positive introduction of students to surgery while challenging stereotypes. It provided a 
better understanding of surgery, enabling students to consider this field, and potentially influencing their residency application.

\section{DP 17-7 Review of the Essential Skills}

Program (ESP) Pilot: A Quality

Improvement Project for Developing

\section{Surgical Skills}

Graham Kasper University of Toronto, Saly Halawa University of Toronto, Shirley Xue Jiang University of Toronto, Caterina Masino University of Toronto, Allan Okrainec University of Toronto

Background/Purpose: Acquiring the technical skills necessary to perform surgery is crucial to achieving competency as a surgical resident. However, no formal surgical skills training is offered in preclerkship curricula. The Essential Skills Program (ESP) is a peer-assisted learning (PAL) program developed to increase pre-clerkship exposure to surgical skills.

Summary of the Innovation: We used the Canadian Institutes of Health Research Knowledge-To-Action (CIHR-KTA) cycle for our program evaluation. Thirtythree medical students completed the program over one year. Using a 3:1 student:tutor ratio, learners were taught over two sessions, one week apart. Skills taught included the subcuticular interrupted suture (SIS) and the Fundamentals of Laparoscopic Surgery (FLS) intracorporeal suture (IC). Performance of IC and SIS were recorded. Students' performances were graded at baseline and at program completion using Global Rating Scales (GRS) and time to completion. Suturing scores were assessed via paired T-tests. Post-program feedback was assessed via survey. IC scores increased significantly between sessions one and two, from a mean time to completion of 417 seconds to 291 seconds. Mean SIS GRS scores increased significantly, from $57.8 \%$ to $71.3 \%$, and time to completion improved significantly as well, from 576 seconds to 452 seconds. Survey results revealed that participants were motivated to improve technical skills for clerkship (94\%) and felt more confident in their technical ability (93\%) after completing the program.

Conclusion: ESP is positively received by peer tutors and participants. This pilot program provides a potential framework for expanding surgical skill exposure to pre-clerkship students and implementing it within formal medical education

\section{DP 17-8 A logic model for IMed: An Internal Medicine Summer Exploration Program for Pre-Clerkship Students}

Alexandra Kobza University of Ottawa, Kaitlin Endres University of Ottawa, Katina Zheng University of Ottawa, Sarah Elias University of Ottawa, Mimi Deng University of Ottawa, Aimee Li University of Ottawa

Background/Purpose: Medical students often have a difficulty selecting a Residency training program as the application deadline predates exposure to all departments. At the University of Ottawa, the sixweek internal medicine rotation is entirely on the inpatient general internal medicine ward. As medical students with a particular interest in the specialty, we identified a need to increase exposure to various subspecialties of internal medicine in order to enrich our understanding of the field. This led us to create a two-week summer program that would give preclerkship students such exposure to assist with the Residency match.

Summary of the Innovation: The two-week summer program for pre-clerkship students involved morning observerships, lunchtime career talks, and afternoon workshops in multiple subspecialties of internal medicine. The morning shadowing gave students a sense of common presentations in the subspecialty, the career talk was an opportunity to discuss lifestyle, the job market and opportunities in the field, whereas students practiced hands-on procedures in the afternoon such as ultrasound imaging of joints in Rheumatology. By the end of the program, students had a taste of nine different subspecialties of Internal Medicine. The program was inaugurated in June 2018 with 18 students participating.

Conclusion: Overwhelmingly participants had positive feedback with regards to the program and felt that they were more prepared to make future decisions about Residency. We created a logic model in order to illustrate our program design and intended outcomes. Our hope is that our comprehensive model will facilitate the creation of similar programs at other institutions. 
DP 18-1 The use of video-based material as preparation for Problem-Assisted Learning (PAL) during the Internal Medicine Clerkship Rotation at the University of Ottawa Medical School

Michelle Turcotte University of Ottawa, Chloé Thabet University of Ottawa, Melissa Phuong University of Ottawa, Glara Rhee McMaster University, Phillip Tsang University of Ottawa, Olwyn Foley Université de Sherbrooke, Ryan Gotfrit University of Ottawa, Laura Zuccaro University of Toronto, Vladimir Contreras-Dominguez University of Ottawa, Robert Bell University of Ottawa

Background/Purpose: During the University of Ottawa's Internal Medicine clerkship rotation students attend small-group Problem Assisted Learning (PAL) sessions reviewing various topics, assisted by a faculty expert. However, due to the heavy demands of this rotation, many students have difficulty preparing for the sessions, leading to valuable in-session time spent reviewing the basics rather than exploring the topic in greater depth. The objective of this student-led initiative was to determine whether providing students with video content tailored to PAL objectives would allow students to better prepare for and optimize their learning during PAL sessions.

Summary of the Innovation: Short overview videos covering high-yield topics were made for 4 PAL sessions. Using a flipped classroom approach, students were able to use these to review basic information prior to their sessions. Following each session, students completed a survey assessing the utility of the videos. Self-reported knowledge levels and preparedness for the PAL sessions before and after viewing the videos were also assessed.

Conclusion: Results ( $n=21)$ suggest that preparatory videos are welcomed by students as an efficient way to prepare for sessions. Thematic analysis revealed that respondents found the videos engaging and presented in a concise and logical manner, but would have further benefitted from a greater number of clinically-relevant examples. Comparison of students' self-assessed knowledge of the PAL topic demonstrated a significant increase after viewing the video compared to before viewing the video $(p=$ 0.00048). Next steps involve modifying existing videos based on feedback from students and implementing this innovative study tool into the curriculum for future cohorts.

DP 18-2 To Lecture or Not to Lecture, That is The Question! Modern Medical and Nursing Student Perceptions Regarding Lectures and Lecture Attendance at the University of Ottawa

Safaa El Bialy University of Ottawa, Mohammad Jay University of Ottawa, John Leddy University of Ottawa, Christopher Ramnanan University of Ottawa, Yamilee Hebert University of Ottawa, Dalia limor Karol University of Ottawa, Neraj Manhas University of Ottawa

Background/Purpose: While the lecture has been a core content delivery method in healthcare profession education; lecture attendance has decreased. Our objective was to define medical and nursing student perceptions regarding lectures and lecture attendance

Methods: Second year medical students (in Spring 2018) and second year nursing students (in Fall 2018) were requested to answer a 10-item survey (consisting of Likert style, multiple choice, and short answer questions).

Results: 110 medical students (response rate 35\%) and 85 nursing students (response rate 40\%) participated in the survey. The top reasons why medical and nursing students attended lectures respectively included: "lectures were mandatory" (81.8\% and 69.9\%), "socializing with peers" $(68.2 \%$ and $28.9 \%$ ), and "professor emphasis on important learning objectives" (67.1\% and 91.6\%). The top reasons for students included the perception that the lecture format was not effective (63.5\% and $66.3 \%)$, preference for using lecture recordings (63.3\% and $18.1 \%)$, and that lecture content was perceived to be of low relevance to the exam (37.5\% and $33.7 \%$ ), and the lectures were scheduled early in the morning (36.5\%and $24.1 \%$ ). Overall, $64.6 \%$ of medical students and $63.4 \%$ of nursing students agree that traditional lectures are an effective way of learning. 
Conclusion: The majority of medical and nursing students perceive that attending lectures still has value in terms of their learning, although medical students also state value for learning via lecture recordings. The emphasis on exam-relevant information and on student engagement are perceived by both medical and nursing students to be methods to enhance lecture attendance.

\section{DP 18-3 Fostering Intergenerational} Education: An Experiential Learning Program for Medical Students and Older

\section{Adults}

Rebecca Correia McMaster University, Lindsay Klea McMaster University, Graham Campbell McMaster University, Andrew Costa McMaster University,

Background/Purpose: As the Canadian population ages, there is a need for more geriatric care physicians. "Make a New Old Friend" is an intergenerational, experiential learning opportunity for medical students with objectives to: 1) Increase competencies in caring for older adults; 2) Enhance communication skills; and 3) Explore career pathways in geriatric medicine.

Summary of the Innovation: The Waterloo Regional Campus of the McMaster University DeGroote School of Medicine implemented two iterations of "Make a New Old Friend" in 2018 and 2019, enrolling a total of eight students. Pre-clerkship students were primarily recruited due to their geographic stability. Students are matched with older adults living at a local Long Term Care (LTC) residence and faculty mentors who practice geriatric medicine. Meetings with older adults happen on a monthly basis to facilitate relationship-building. These in-person visits occur at the LTC residence to provide exposure to daily living of older adults. Students meet with faculty mentors to discuss their experiences and may arrange a shadow day. Students complete an attitude and interest survey to facilitate the matching process. Evaluations based on Kolb's Experiential Learning Cycle are completed by students and faculty mentors to consolidate learning outcomes. The volunteer coordinator from the LTC residence also conducts brief evaluations with the older adults.
Conclusion: Students report high levels of satisfaction with this learning experience in follow-up interviews. The research team is investigating strategies to increase participation, and collecting feedback to guide future iterations. Intergenerational, experiential learning opportunities can be facilitated across levels of education and disciplines to support our aging population.

DP 18-4 Assessing performance of Endocrinology \& Metabolism Residents after wearing diabetes technology: A matter of experience

Linda Wang University of Ottawa, Stephanie Dizon University of Ottawa, Amel Arnaout University of Ottawa

Background/Purpose: As medical devices become increasingly important in the management of chronic diseases such as Type 1 diabetes (T1DM), both technological proficiency and understanding the patient experience are crucial for patient-centered care. However, effective methods of delivering a diabetes technology curriculum have not been described.

Summary of the Innovation: We implemented a "Diabetes Bootcamp" in which trainees lived a simulated patient experience by wearing diabetes technology. The purpose was to promote experiential learning and to ascertain how this impacted practical skills in managing insulin pumps and continuous glucose monitors (CGMs). The "bootcamp" consisted of a 7-day period of wearing devices. Residents completed a pre-test attempting important day-today tasks of patients and clinicians, using an insulin pump with predetermined settings. After brief training, they were sent home wearing a saline pump and CGM, and instructed to adhere to guidelinebased practices of a T1DM patient. After 1 week. they completed a post-test, feedback survey, and semistructured group discussion reflecting on their experience, interpreting their reports facilitated by experts.

Conclusion: All six residents reported increased confidence using technologies $(4.50+/-0.55$ vs. 2.67+/-1.03 on a 5-point Likert-like scale, $p=0.006$ ). Significant objective improvements occurred in 
modifying settings ( $92 \%$ vs. $71 \%, \mathrm{P}=0.042$ ) and overall performance ( $92 \%$ vs. $82 \%, \mathrm{P}=0.007$ ) on the post-test. Residents expressed insights into the lifestyle of T1DM, challenges of carbohydrate-counting, common logistical issues, and burnout in diabetes. An experiential learning program with hands-on assessment is an effective way to increase practical skills and empathy for patients with chronic disease.

DP 18-5 Mixed methods analysis of an automated e-mail audit and feedback intervention for fostering (emergency)

\section{physician reflection}

William Kennedy University of Calgary, Daniel Andruchow University of Calgary, Shawn Dowling University of Calgary, Kevin Lonergan Alberta Health Services, Tom Rich Alberta Health Services, Catherine Patocka University of Calgary

Background/Purpose: Physician reflection requires personalized, timely and growth-oriented feedback. Iterative learning from multiple low-pressure events can be personalized to target areas of weakness and show sequential growth. Since emergency physicians typically work individually to deliver episodic care, opportunities for them to obtain iterative feedback on their clinical performance is often limited. Our study sought to evaluate whether physician reflection is facilitated through the $72 \mathrm{hr}$ re-admission alert received by emergency physicians in the Calgary zone.

Methods: This mixed methods realist evaluation consisted of two sequential phases: an initial quantitative phase examining the general features of 72-hr readmission alerts sent over a 1-year period (4024 alerts from May 2017-2018) and a subsequent qualitative phase involving 17 semi-structured interviews to generate "context-mechanismoutcome" (CMO) statements to guide refinement of our program theory.

Results: CMO statements revealed emergency physician stakeholders were concerned that the alert impacted personnel decisions, changed patient return expectations and didn't involve consulting services. Physicians, who didn't believe alerts were involved in personnel decisions, were more likely to pursue balanced reflection/acquisition after each alert when receiving illness related returns. Conversely, physicians, who believed alerts were involved in personnel decisions, were more likely to defensively change their practice. Commonly cited areas of improvement were the ability to personally adjust time criteria for alerts and involving consulting services in feedback.

Conclusion: Under certain conditions the individualized, timely feedback from a 72-hr readmission alert appeared to facilitate physician reflection.

\section{DP 18-6 Conception et évaluation d'une} formation sur les principes de « Choisir avec soin $"$

Lyne Pitre University of Ottawa, Chantal D'aoustBernard University of Ottawa, Anne-Marie Friesen University of Ottawa, David Adjo University of Ottawa

Background/Purpose: Au Canada, les coûts liés à la santé sont élevés. Dans les CanMEDS, la gestion des ressources est une compétence que devrait développer un médecin. Les étudiants en médecine doivent donc être sensibilisés à l'aspect financier des soins de santé. Ce projet a été réalisé dans un contexte d'éducation médicale à l'université d'Ottawa. II visait à concevoir une formation portant sur " les tests inutiles " et vérifier l'effet de cette formation sur l'acquisition de connaissances des étudiants.

Methods: Un MAA sur " les tests inutiles " a été conçu puis révisé par des experts. La formation a été offerte aux étudiants de troisième année suivant un modèle de classe inversé. Les connaissances des étudiants ont été évaluées grâce à un questionnaire auto administrée pré et post test. La comparaison des scores moyens obtenue avant et après la formation a été effectuée grâce au logiciel d'analyse de données quantitatives SPSS.

Results: 48 étudiants donc $73 \%$ de femmes ont participé au projet. $76 \%$ avaient un âge compris entre 22-25 ans. Le score moyen obtenu par les étudiants avant la formation était de 9,7 sur 20 contre 11,7 après la formation. Les différences entre ces scores étaient statistiquement significatives ( $t:-3,45$ et $p$ : 0,0013). 
Conclusion: Un modèle de classe inversée incluant un MAA sur les tests inutiles pourrait contribuer à accroitre les connaissances des étudiants. Un deuxième post test sera réalisé deux mois après la formation afin de vérifier la rétention.

\section{DP 18-7 Reflexi-Vite: A practical tool for} fostering reflexivity in family medicine

Marie-Claude Tremblay Université Laval, Anne Guichard Université Laval, Julien Quinty Université Laval, Christian Rheault Université Laval, Chantal Gravel Université Laval, Marie-Claude Tremblay Université Laval

Background/Purpose: In the last decade, reflexivity has emerged as a key concept in family medicine as evidenced by its increasing integration in competency statements and frameworks in the field. However, the growing variety of terms and definitions related to reflexivity has caused vagueness and hindered the concept's full application in training and professional practice. In 2017, the directors of Université Laval's Family Medicine Residency Program recognized such issues in integrating reflexive activities within their program. They highlighted the need to better equip and support program teachers regarding reflexivity.

Summary of Innovation: Our team co-developed a tool to support reflexive role modelling by Université Laval's Family Medicine Residency Program teachers. "Reflexi-Vite" was developed through a rapid review of literature in family medicine ( $n=64$ articles) and a needs assessment of clinician teachers ( $n=96$ ) affiliated with the program. Reflexi-Vite presents four types of reflexivity (i.e. clinical reflexivity, professional reflexivity, relational reflexivity and socially responsible reflexivity) in a concise way with associated definitions, goals, processes and example question prompts for each reflexivity type. The tool is available in PDF and interactive PDF (optimized for smartphone) formats.

Conclusion: Our assessment shows a high uptake rate of Reflexi-Vite in the residency program, and there is current interest to adopt it within other health sciences programs in Québec including nursing sciences, physiotherapy and other medical specialties. Reflexi-Vite is crucial for fostering professionalism, a sense of social responsibility and continued professional improvement among future health professionals.

DP 18-8 Reflection in healthcare learner: Should it be evaluated and how should it be evaluated?

Cassandra Préfontaine Université de Montréal, Chantal Lemire Université de Sherbrooke, Hélène Corriveau Université de Sherbrooke, Marie-Josée April Université de Sherbrooke, Isabelle Gaboury Université de Sherbrooke

Background/Purpose: Reflection - for learning and improving practice - is a part of the curricula for many health professions. There is an ongoing debate among scholars, educators and trainees about whether or not healthcare trainees should be evaluated on their reflective process. Consequently, few tools are available to educators who choose to evaluate the reflective process. This project aimed to: 1) document the academic debate on evaluating reflection, and 2 ) to review evaluation tools available in the literature.

Methods: First, a meta-interpretation of the arguments in favor and against evaluating the reflective process was performed. Second, a scoping review of the literature was conducted to find evaluation tools.

Results: Four arguments in favor of evaluating the reflective process emerged: 1 ) evaluation emphasizes the importance of reflection; 2) it ensures adequate depth of reflection among trainees; 3 ) it provides trainees with feedback on the reflective process; and, consequently, 4) it supports trainees' personal and professional development. Conversely, the four arguments against evaluation were related to: 1 ) how evaluation limits the learning process; 2 ) the amount of resources required to conduct evaluations, 3 ) the anxiety generated by vague evaluation criteria; and 4) low validity and poor psychometric properties of evaluation tools. In the literature, 31 evaluation tools - based on various theoretical models - were identified. Across the tools, 11 components to evaluate the reflective process were identified. None of the tools encompassed all components, but most included 3 to 5 . Validity and reliability assessments, 
with reasonable scores, were presented for most tools.

Conclusion: Beyond whether or not the reflective process should be evaluated, it is important to consider how the culture of evaluation in higher education affects evaluation of the reflective process. The choice of tools to evaluate reflection should take into consideration the context of use. Further research is needed with respect to the psychometric values of existing tools.

\section{DP 18-9 Factors That Shape Decision}

Making Around Resuscitation Preference: A Scoping Review with Implications for Clinical Training

Kristen Bishop Western University, Hasan Hawilo Western University, Ravi Taneja Western University, Mark Goldszmidt Western University

Background/Purpose: Predicting factors that shape decision making around resuscitation preference for adults has been the subject of extensive study. However, existing reviews summarizing this literature are inadequate for supporting clinical training. In part because the underlying issues that need to be explored during a conversation have not been fully explicated. The purpose of this scoping review is to comprehensively and systematically synthesize existing empirical research on the factors that shape, and the reasoning behind how and why adults make particular decisions about resuscitation preferences for themselves and as substitute decision-makers (SDMs).

Methods: Arksey and O'Malley's (2005) five-stage framework was used to describe the current knowledge base related to how and why adults make particular decisions around resuscitation preference. Of the 4,019 articles retrieved from the search of 4 databases and through citation tracking, 30 articles met the inclusion criteria and were included in this review.

Results: Identified studies could be categorized across 3 categories: explicit exploration of resuscitation preference reasoning; implicit exploration; and correlational exploration. Across the study types, none fully addressed the broader issue of how and why patients or SDMs make particular choices in relation to resuscitation for themselves and others.

Conclusion: To date, there continues to be a paucity of studies exploring the underlying issues that should be explored during a resuscitation preferences conversation with patients and their SDMs. This gap presents an ongoing challenge for training health professionals in how to effectively elicit resuscitation preferences. It also flags important areas for future research.

DP 19-1 Comparison of the English and French versions of the CASPer $^{\circledR}$ Test in a bilingual population

Lemay Genevieve University of Ottawa, Christopher Zou Altus Assessments, John Leddy University of Ottawa, Meghan McConnell University of Ottawa, Patrick Antonacci Altus Assessments

Background/Purpose: The University of Ottawa MD program has two different streams to which candidates may apply: a francophone stream and an anglophone stream. As the admissions office receives applications in both French and English, they are required to ensure that the tools used to assess candidates are psychometrically equivalent across both streams. CASPer is a standardized test they recently adopted to assess the non-cognitive competencies of applicants and is offered in both English and French. The objective of this study is to compare the psychometric properties of the English and French versions of CASPer.

Methods: We collected data from all CASPer testtakers across three cohorts ( $n=12,463$; entry 2016, entry 2017, entry 2018). We first compared the difficulty of the test between the French and English version using proxy indicators (i.e., time to completion, typing speed). We then compared the psychometric properties of the two versions based on their internal-consistency reliability and applicant acceptability.

Results: There were some indications that the French version may be slightly more difficult than the English version of the CASPer test. However, it is unclear whether this difficulty is due to the difficulty of the individual test items or to differences in the 
characteristics of the cohort. Nevertheless, a comparison of the psychometric indicators suggests that both French and English versions of CASPer are psychometrically sound and equivalent.

Results: Although CASPer scores cannot be directly compared between the English and French versions, the psychometric properties of the assessment were retained across the two versions. These results provide preliminary evidence that the psychometric strengths of the English version of CASPer likely extend to the French version of the assessment.

Conclusion: Although CASPer scores cannot be directly compared between the English and French versions, the psychometric properties of the assessment were retained across the two versions. These results provide preliminary evidence that the psychometric strengths of the English version of CASPer likely extend to the French version of the assessment.

\section{DP 19-2 Assessing the reflection process: Medical students' perceived consequences}

Ann Graillon Université de Sherbrooke, Joanie Poirier Université de Sherbrooke, Kathleen Ouellet Université de Sherbrooke, Valérie Désilets Université de Sherbrooke, Marianne Xhignesse Université de Sherbrooke, Christina St-Onge Université de Sherbrooke

Background/Purpose: Helping trainees develop their reflection skills is a challenge faced by many educators. One means to give value to the development of this ability is through credited courses and assessment. Yet, the assessment of reflection is fraught with challenges and potentially negative consequences. Based on an extensive literature review, we created a pass-fail assessment wherein mentors assess trainees' participation in a 4year longitudinal course that aims to promote reflection on their professional identity and practice.

Methods: We conducted a qualitative descriptive study using semi-structured interviews with medical students to document their perceptions, and thus potential consequences, of the assessment for the reflection course. The thematic analysis was informed by Braun \& Clarkes' methodology. One author (JP) coded the interviews, with checking by other team members (KO, CSO). Three team members (CSO, KO, $\mathrm{JP})$ discussed the coding structure extensively until they arrived at an interpretation of the data. Interpretation of findings was presented to the entire team and discussed until a consensus was achieved.

Results: We conducted interviews with 21 1st year medical students and 6 2nd year medical students. We identified three main themes (subsequently declined into subthemes) in our data corpus: 1consequences associated with the assessment of the reflection process (vs content); 2 - consequences due to a pass-fail assessment; and 3- consequences associated with the mentors' dual role.

Conclusion: Assessment of the reflection process, even when content is not assessed, may entail positive and negative consequences. When planning an intervention aimed at developing reflection skills, these potential consequences should be considered.

DP 19-3 The Compatibility Principle: On Philosophies in the Assessment of Clinical

\section{Competence}

Walter Tavares University of Toronto, Ayelet Kuper University of Toronto, Mahan Kulasegaram University of Toronto, Cynthia Whitehead University of Toronto

Background/Purpose: A range of often implicit philosophical positions (e.g., post-positivism, constructivism, pragmatism) now underlie assessments programs in health professions education (HPE). Challenges may arise when assumptions and commitments informing competence, assessment activities and justification/validity are not sufficiently considered in assessment design or are in conflict. This includes a logical incompatibility leading to varied or difficult interpretations of assessment results or perpetuating an "anything goes" approach.

Methods: This narrative study examined assessment literature in HPE to explore the plurality of philosophical positions informing components of assessment including competence, assessment strategies, and justifications/validity, as well as the need to consider coherence between them. We also examined philosophical tensions within health professions assessment research and in other fields 
(e.g., mixed methods research) to derive implications for assessment practice and scholarship.

Results: There is a degree of indeterminacy in the nature of, definition and meaning given to competence, assessment strategies, and activities and validity/validation as a result of the different philosophical positions. These guide assessment differently and translate into different practical choices and interpretations about the quality and appropriateness of the assessment programs. Logical coherence can no longer be assumed. A "compatibility principle" which obligates attention to these issues of assumptions and commitments may help support assessment designs.

Conclusion: Assessment in the health professions is broadening in its use of philosophical positions, which affect the "state of play". This creates the potential for a logical incoherence that can undermine assessment programs but may be overcome with attention to the alignment between underlying commitments and assumptions.

DP 19-4 Defining a set of Palliative Care Entrustable Professional Activities based on Priority Topics and Key Features

Jacqueline Hui University of Calgary, Keith WycliffeJones University of Calgary

Background/Purpose: Twelve validated Palliative Care Entrustable Professional Activities (PC-EPAs) outline what Palliative Care (PC) residents should be entrusted to perform by the end of postgraduate training. For Family Medicine Enhanced Skills Palliative Care Programs (FM-ES PC), defining the specific competencies that comprise PC-EPAs for assessment has been challenging as many frameworks exist, including CanMEDS-FM and the College of Family Physicians of Canada (CFPC) Evaluation Objectives' Priority Topics and Key Features (KFs). We sought to clarify assessment expectations for these PC-EPAs by: 1) Defining PCEPAs based on the CFPC PC Enhanced Skills Priority Topics and KFs by mapping and focusing chosen KFs as core competencies. 2)More accurately describing what competent performance looks like for each EPA.

Summary of the Innovation: Our FM-ES PC Residency Program Committee members were assigned 1-2 PC-
EPAs and asked to choose KFs considered to be essential core competencies for their assigned EPA(s). 12 PC-EPAs were mapped to selected PC KFs for our FM-ES PC Program. The average number of KFs per PC-EPA = 37 (3-107). Each KF was mapped (no "orphaned" KFs). Average number of PC-EPAs mapped per Key Feature = 3.5 (1-7). Mapping a set of chosen KFs as core competencies that define each EPA can validate the PC-EPAs and the CFPC KFs themselves, as appropriate, granular-level descriptors of competent performance by a FM-ES PC resident. Sufficient sampling of performance and enhancement of these selected KFs over time will be used to determine if a resident is entrustable in each EPA by the end of training. A nominal group technique will be used to establish consensus.

Conclusion: This assessment approach may be beneficial to other FM-ES Programs by providing a suggested framework for summative and completion decisions.

DP 19-5 Specialist assessment activities: The interface of learning, change, and discussion

Jocelyn Lockyer University of Calgary, Jocelyn Lockyer University of Calgary, Craig Campbell The Royal College of Physicians and Surgeons, Shanna DiMillo The Royal College of Physicians and Surgeons

Background/Purpose: Fellows and participants in the Royal College of Physicians and Surgeons of Canada Maintenance of Competence (MOC) program are required to participate in assessment activities. This study examined data for five different specialties to identify variation in assessment activities; examine differences in the resultant learning; assess the frequency and type of changes that were planned; and assess the association between learning and change and between planned changes and discussion.

Methods: Data from MAINPORT were categorized and analyzed descriptively. Chi-squared tests examined associations.

Results: 6,063 assessment activities from 2,854 physicians were provided by anatomical pathologists, cardiologists, gastroenterologists, ophthalmologists, and orthopedic surgeons. There were differences in 
the frequency of learning by discipline and assessment type. The most frequent assessment activities across all specialties were self-assessment programs $(n=2122)$, feedback on teaching $(n=1078)$, personal practice assessments which the physician did themselves ( $n=751)$, annual reviews $(n=682)$, and reviews by third parties $(n=661)$. Learning occurred for $93 \%(n=5668)$ of the activities and was associated with change. For 2126 (35\%) activities, changes were planned. A total of 1145 (18.8\%) of activities involved a discussion with a peer or supervisor and these activities were more likely to result in plans for change.

Conclusion: While specialists engaged in many types of assessment activities, there was variability in learning and plans for change. Organizations requiring participation in assessment activities might prioritize those requiring a discussion about data as they are more likely to facilitate planned change.

\section{DP 19-6 Pharmacy students complete} formative and summative entrustment assessments (EPAs) to appraise self and peer ability to perform unsupervised in the self-care community workplace

Debra Sibbald University of Toronto

Background/Purpose: Pharmacists are entrusted to assess, manage and educate patients who choose to self-select treatment for minor ailments. Prospective or retrospective entrustment assessments are customarily rated during training by clinician observers. Undergraduates should develop EPA selfregulation skills for working unsupervised as community pharmacists after licensure.

Methods: Two cohorts of Pharmacy students (2nd year required self-care therapeutics course; 3 rd year elective) performed classroom EPA assessments in their pre-clinical years to foster self-regulation for community practice training and to cultivate confidence. Structured EPAs were created for patient assessment, treatment plans and education using best practice guidelines. EPA rankings were anchored to five levels of supervision. Self and peer formative assessments (pre and post topic) were completed weekly to measure retrospective or estimate prospective entrustment for unsupervised practice.
Pre and post course summative self and peer ratings were also compared. Post elective, students projected the ability to work unsupervised for peers who had not completed this additional optional training course. Perceptions were analyzed from observations, reports, surveys, class discussions and interviews.

Results: Student reflections on pre vs post ratings highlighted feelings of increased confidence in estimated ability to perform in practice. Pre vs post self and peer ratings all showed advancements. Elective course students felt more prepared to work independently having had additional training.

Conclusion: Students valued this opportunity to perform self and peer EPA assessments as training for self-regulated practice. It heightened their commitment to their professional role and stressed the importance of monitoring their entrustment expertise when working unsupervised.

DP 19-7 Are there gender differences on physicians' reflection and actions taken to improve practice gaps?

Diana C Sanchez-Ramirez University of Manitoba, Hannah Smith University of Manitoba, Dori Rainey University of Manitoba

Background/Purpose: Although numerous studies have examined the effectiveness of peer-assisted debriefing of multisource feedback (MSF) reviews on physician improvement, outcome variations between genders, to the best of our knowledge, has not yet been explored. The general objective of this study was to explore whether gender influences physicians reflection on the MSF received regarding their practice and how they choose to respond to this feedback.

Methods: This retrospective cross-sectional study examined MPAR peer-assisted reflective surveys completed by Manitoba physicians between 2011 and 2018. Survey responses were categorized according to the CanMEDS competencies and assessed to determine if there were gender differences in physicians' perceived feedback and changes implemented. 
Results: One hundred and sixty-nine physicians submitted the reflective survey (71\% males). Physicians expressed receiving (unpleasant) unexpected/surprising comments mainly in regards of their roles as an expert (28\%), communicator (26\%), collaborator (25\%) and manager (20\%); and to a lesser extent to their roles as a professional (11\%), scholar (7\%) and health advocate (3\%). A higher percentage of females (88\%) who received a comment related to their role as a professional implemented a change for improvement in that area compared with their male counterpart ( $88 \%$ vs $33 \%$, $\mathrm{p}=0.02$ ). No other gender differences were found in physicians' perceived feedback or changes implemented.

Conclusion: Results suggested that there were no gender differences on physicians' reflection on their practice after receiving MSF. However, female physicians were more inclined to incorporated changes to improve their competence as a professional.

\section{DP 19-8 Effect of Station Format on the} Psychometric Qualities of Multiple MiniInterviews

Jean-Sébastien Renaud Université Laval, Martine Bourget Université Laval, Christina St-Onge Université de Sherbrooke, Kevin Eva University of British Columbia, Walter Tavares University of Toronto, Alexis Salvador Loye Université Laval, JeanMichel Leduc Université de Montréal, Matthew Homer University of Leeds

Background/Purpose: Multiple Mini-Interviews (MMI), a tool assessing non-academic skills, play a major role in the selection of future physicians who will provide health care to the population. Researchers interested in the psychometric qualities of MMIs have demonstrated the effects of factors related to examiners, candidates, and stations. The effect of station format used (interview or role-play) remains overlooked despite differences in the way skills are assessed, and the fact that station format is a factor over which programs have considerable control. This study aimed to compare the psychometric qualities of these two station formats.
Methods: Scores from the 2010-2017 Quebec French Integrated MMI $(n=11,173)$ were used to compare difficulty and discrimination as well as the reliability of the two station formats (276 interview, 246 roleplay). Results from the 2011-2014 MMIs and the average clerkship rotation score of Université Laval's MD students $(n=462)$ were used to compare the predictive validity of the two station formats using mixed linear regression.

Results: Interview stations had a higher mean score than role-play stations, with a small effect size ( $M=$ $21.3(S D=3.1)$ vs. $M=20.7(S D=3.4), p<0,05, d=0,2)$. The two station formats were not statistically different in their discrimination index (0.32 vs. 0.33 ) or reliability coefficient. Both formats were weakly associated with the clerkship average score $(R 2=0.05$, $\mathrm{p}<0.05)$.

Conclusion: Interview and role-play stations have similar psychometric qualities suggesting that programs should select station format based on match to the personal qualities for which they are trying to select rather than statistical characteristics.

DP 19-9 Do high grades prior to MD studies lead to higher academic achievement during the MD curriculum and higher scores on licensing examinations?

Margaret Henri Université de Montréal, Robert Gagnon Université de Montréal, Christian Bourdy Université de Montréal, Jean-Michel Leduc Université de Montréal, Geneviève Grégoire Université de Montréal

Background/Purpose: Until 2018, at our institution, student applying to the MD program were selected for admission based on 2 criteria: a standardized grade point average (GPA) and a multiple mini interview (MMI) score. The GPA accounted for $50 \%$ of the total score for admission. With this study, we wished to answer this question: is the standardized GPA a reliable performance predictor for grades during the MD curriculum, and for the Medical Council of Canada Qualifying Examination Part I (MCCQE I)?

Methods: From 2008 to 2015, data were prospectively collected from all students entering the 
MD program. Data included: GPA from pre-university or university studies, mean grades in years 0 (premed) 1 and 2, grades from project-based learning and courses throughout MD studies (eg: basic anatomy, digestion and nutrition, heart and circulation), endof-curriculum written exam and OSCE scores, and MCCQE I results. Pearson's coefficient was used to analyse the relationship between these scores.

Results: GPA showed weak correlation to year 0 $(r=0.296, n=646, p<0.001)$, year $1(r=0.231, n=1113$, $p<0.001)$, and year $2(r=0.258, n=1098, p<0.001)$ mean grades. GPA presented no correlation to endof-curriculum written examination scores $(r=-0.034$, $\mathrm{n}=579, \mathrm{p}=0.416)$ or OSCE scores $(\mathrm{r}=0.008, \mathrm{n}=838$, $\mathrm{p}=0.813)$. GPA also correlated poorly $(r=0.202$, $n=1805, p<0.001$ ) with results at MCCQE I.

Conclusion: While high grades are still considered an important standard for admission to our MD program, they do not demonstrate a strong correlation to high-stakes, end-of-curriculum and licensing examinations. Further analysis will help to determine if different weighing assigned to GPA and MMI scores, or if additional criteria for admission to our MD program would yield improved results.

\section{DP 20-1 Practical Approaches to Obesity Management: Interprofessional Care of Patients with a Complex Disease}

Boris Zevin Queen's University, David Barber Queen's University, Robyn Houlden Queen's University, Eleftherios Soleas Queen's University, Lynn Roberts Queen's University, Richard van Wylick Queen's University, Katie Evans Queen's University, Nancy Dalgarno Queen's University

Background/Purpose: Obesity and its related diseases are prime public health issues. In response to this need, a group of Queen's health education researchers and healthcare providers partnered with the Queen's Centre for Studies in Primary Care to develop an interprofessional professional development program on management of patients with obesity.

Methods: The research findings were integrated in planning a one day program developed around a case study of a patient struggling with obesity. Obesity management topics were explored by an interprofessional team including a registered dietician, psychiatrist, exercise kinesiologist, endocrinologist, general internist, bariatric surgeon, family physician, and nurses. The resulting program educated 35 primary care providers (PCPs) to proactively manage patients with obesity using an array of available behavioural, psychological, pharmacological, and surgical approaches to improve healthcare outcomes in patients with obesity.

Results: Over $90 \%$ of evaluations had perfect scores for confidence and increase in knowledge with the remainder being overwhelmingly positive. This universally positive reception of the content and its delivery, spurred subsequent CPD offerings with additional focus on interdisciplinary case studies. There were significant differences between participants in their level of confidence to engage in difficult medical conversations related to obesity, and in their knowledge of approaches to management of patients with obesity.

Conclusion: The richness of the learning, the practicality of the topic, and the interprofessional focus of this program offers an exemplary model for other health education institutions interested in offering this program to address current challenges with management of patients with chronic diseases.

DP 20-2 Twelve Tips: Implementing and Sustaining a Successful Interprofessional Simulation Educational Program

Stephen Miller Dalhousie University, Diane MacKenzie Dalhousie University, Gail Creaser Dalhousie University, Kim Sponagle Dalhousie University

Background/Purpose: Interprofessional education (IPE) is fast becoming a mainstay of health professions curricula. Well-designed simulationbased education can enhance IPE, where students and faculty are afforded rich experiential learning opportunities to learn about, from, and with each other.

Summary of the Innovation: Our interprofessional healthcare educational team at Dalhousie University focuses on learnings from a robust interprofessional (IP) simulation-based education (SBE) program that has had multiple iterations. The integrated curriculum 
is facilitated by IP faculty and provides profession specific and interprofessional collaborative (IPC) team training to 400 students from six professions. The Collaborative Stroke Interprofessional curriculum is a two-part educational series comprising four hours of student interaction in IP teams. The first session is a high fidelity table top simulation incorporating best evidence and practice guidelines in stroke care. This session is preceded by flipped classroom modules for student pre-learning. The second session is a simulated stroke clinic with simulated patients and students working through scenarios in IP teams. The program is well-received and has demonstrated changes in knowledge and attitudes. Our IPC team will relate ways to make these IP programs successful in your institution, achieving significant IPE outcomes and administrative efficiency

Conclusion: Our IP curriculum has demonstrated significant longevity and educational outcomes that can be replicated in your institution. Challenges and barriers will be discussed, as well as providing pedagogical suggestions for overcoming these concerns, so that in one's own work sphere attendees may develop a program that is successful and sustaining in its scope.

DP 20-3 Interprofessional Collaboration in the Real World - Patient's Medical Home as a foundation for success

Amanda Condon University of Manitoba, Dana Turcotte University of Manitoba, Jessica Clendenan University of Manitoba, Margaret Rauliuk Athabasca University

Background/Purpose: Clinical care models, including the Patient's Medical Home (PMH), identify interprofessional teams as integral to optimal patient-centered care. These environments can provide authentic clinical learning opportunities for health professional learners. It is recognized that interprofessional education must ensure training in the context of the work environment so learners can develop the collaborative skills necessary to adapt to the clinical setting. Literature in this area is often focused on evaluation of select interventions or activities, with less emphasis on foundational structures and team culture that need to be in place within a clinical setting to optimize authentic learning experiences.

Summary of the Innovation: An interprofessional primary care team in Winnipeg, Manitoba, Canada, provides comprehensive team-based care, in keeping with the PMH model, and regularly includes interprofessional learners. This poster describes the foundational structures and clinical activities within this team that support interprofessional learning and role modeling for health professional learners, as well as highlighting opportunities for ongoing optimization.

Conclusion: Community-based primary care is well situated to provide authentic clinical interprofessional learning experiences for learners across health professions. Existing foundational culture and workflow are key to optimal collaborative experiences in this environment. This foundation provides seamless incorporation of students into the existing team and thus, meaningful and authentic clinical experiences. Using the PMH model to evaluate local adaptation can identify opportunities for authentic IP learning experiences.

\section{DP 20-4 Interprofessional Identity: Are we all on the same plate?}

Wendy A Stewart Dalhousie University

Background/Purpose: Healthcare disciplines each have their own distinct training, clinical practice framework and professional identity, leading to conflict and tribalism in team-based care. Team based research has focused primarily on critical care settings and interprofessional identity has not been well defined. This study sought to contribute to an understanding of power and hierarchy in a variety of healthcare contexts and develop a concept of interprofessional identity.

Methods: Following Research Ethics Board (REB) approval, eight female health professionals participated in the study: 3 medical disciplines, 4 allied health and 1 from nursing. Participants completed an 8-module course on humanism and professionalism. Pre- and post-course qualitative interviews were conducted. Analysis was completed using phenomenology. From these data, an in-depth 
understanding of team functioning was obtained and a concept of interprofessional identity generated.

Results: Leadership behaviour influenced team functioning and how individuals perceived their role. Team members had agency when their input was valued and disempowered when excluded or their input dismissed. Participants saw the patient differently when part of the team; this shift in thinking was transformative. Each discipline had the same new perspective focussed on the patient's goals and wishes rather than individual discipline goals.

Conclusion: Team-based care continues to have issues of hierarchy and conflict yet can transform thinking around the patient. The proposed concept of interprofessional identity shifts the primary focus to the patient's needs and how each discipline helps meet them rather than approaching care from a discipline perspective. This could change our thinking around interprofessional education, team building and leadership.

\section{DP 20-5 The Power of Professionalism}

Award: Fostering Professionalism in an

\section{Academic Institution}

Ellen M. Friedman Baylor College of Medicine, Larry Laufman Baylor College of Medicine, Joan Friedland Baylor College of Medicine, Anne Gill Baylor College of Medicine, Vandana Shah Baylor Collge of Medicine

Background/Purpose: In an effort to emphasize our institutional appreciation of noteworthy professional behavior, The Center for Professionalism created the Power of Professionalism (POP) Award, which rewards exemplary professionalism and recognizes role models who demonstrate noteworthy actions, such as acts of compassion, empathy, selflessness and teamwork. Acknowledging individuals' noteworthy actions reinforces career satisfaction and decreases burnout. There is an institutional impact because the award documents that caring, professional behaviors matter and our organizational commitment to professionalism. Proactivity validating positive behavior may be more effective than remediating unacceptable behavior in the creation of a professional work environment. Individuals from all campus affiliates and all positions are eligible to receive a POP award, which has brought a sense of community throughout Baylor

Summary of the Innovation: The POP Award is a successful, low cost method for recognition. Recipients include clinicians, basic scientists, medical and graduate students, residents, and staff. To date, 70 nominations have been received and 64 have been rewarded. The 32 awardees still at Baylor received an anonymous survey; 18 responded (53\% response rate). A majority agreed receiving the award improved any sense of professional burnout, $89 \%$ felt the award validated their professional efforts and Baylor's commitment to professionalism, and $83 \%$ felt they were motivated to continuing to demonstrate the highest level of professionalism

Conclusion: Will discuss the lessons learned in the creation of POP award and the measureable positive impact of this award. The POP Award has created an expanding culture, emphasizing positive professional behaviors, publicizing positive role modeling and giving personal and peer recognition

DP 20-6 Promoting the Well-Being and Resilience of Health Professional Learners:

Developing and Implementing an

Interprofessional Healthy Learning

\section{Curriculum}

Camila Velez McGill, Deborah Friedman McGill, Nicole-Ann Shery McGill

Background/Purpose: Health professional learners often experience significant distress that can impair their mental health, decision-making, and ability to thrive in their programs. Besides dealing with a demanding academic curriculum, clinical rotations, and constant evaluations, health professional learners also have to respond to their patients in a professional and compassionate manner. It is critical to equip learners with skills to problem solve and develop resilience within a challenging health care system.

Summary of the Innovation: Since 2016, the WELL Office in the Faculty of Medicine at McGill University has been working to promote the well-being of learners from the School of Physical and Occupational Therapy, Ingram School of Nursing, and School of 
Communication Sciences and Disorders. The WELL Office is proactive in reaching learners through the Healthy Learning Curriculum and providing them with tools to becoming problem solving, critical thinking, and resilient health professionals. Concurrently, we are reactive in offering academic and mental health counselling. Curriculum topics include stress and time management, building resilience, finding positive solutions to negative interactions, managing perfectionism, grit, and leadership. The topics are based on feedback from learners in different forums, curriculum evaluations and exit surveys, and input from leadership, faculty, wellness consultants, and Assistant Dean, Student Affairs. Feedback has been positive and the desire for a longitudinal curriculum continues to grow.

Conclusion: This presentation will share the implementation process of the Healthy Learning Curriculum, the nature of the curriculum sessions and feedback from learners, as well as recommendations for the successful development of a curriculum to foster health professional learners' well-being.

\section{DP 20-7 Création d'une formation par} concordance interdisciplinaire: présentation du processus et leçons

\section{apprises}

Rebecca Maftoul Université de Montréal, Vincent Jobin Université de Montréal, Bernard Charlin Université de Montréal, Alexandre Berkesse Centre d'excellence sur le partenariat avec les patients et le public, Annie Descoteaux Université de Montréal, Caroline Lebel Université de Montréal, Marc Rouleau Université de Montréal, Anouck Sénécal Université de Montréal

Background/Purpose: Le développement professionnel continu en collaboration des équipes de soins est un défi au regard de contraintes du champ d'expertise des professionnels, de temps et des ressources disponibles. Une problématique comme la dysphagie est un domaine où la formation et la cohésion de l'équipe de soins (y compris le patient et son entourage) est essentielle. La formation par concordance est une méthode innovante qui, avec un minimum de ressources, pourrait permettre d'offrir une occasion de développement professionnel en intégrant la complexité d'un tel contexte.

Summary of the Innovation: Nous avons créé une formation par concordance sur la dysphagie qui s'adresse aux professionnels des disciplines impliquées. Les buts: - D'être exposé aux perspectives de chaque discipline impliquée (patient, orthophoniste, ergothérapeute, nutritionniste, médecine et infirmière) - De mieux définir les contributions de chaque discipline De réfléchir aux conséquences de cette compréhension pour sa propre pratique. Cette formation amène à percevoir le raisonnement propre à chaque partie (patient et différentes disciplines). Pour ce faire, nous avons généré des vignettes cliniques à partir de problématiques authentiques. Nous avons ensuite fait appel à un panel composé de professionnels impliqués et de patients avec une expérience en dysphagie. Chaque membre du panel a fourni des réponses pour chacune des vignettes en justifiant ses positions. Nous présenterons les étapes du processus et les défis que nous avons dû relever pour y parvenir.

Conclusion: La formation par concordance permet d'offrir une activité de développement professionnel pour améliorer la collaboration en rendant explicite les perspectives distinctes et complémentaires des membres de l'équipe de soins incluant le patient.

\section{DP 21-1 Francisation du curriculum de médecine M.D. de McGill}

Tin Ngo-Minh McGill, Gilles Brousseau McGill, Michel Leblanc McGill, Fabien Vadnais McGill

Background/Purpose: Le programme de formation en médecine M.D. sera entièrement donné en français au nouveau campus médical délocalisé en Outaouais de l'Université McGill en septembre 2020. Le principal défi est le court délai pour la réalisation de la francisation de tout le curriculum qui demande à la fois efficacité et qualité de la traduction. La tâche est particulièrement ardue en raison du jargon et des acronymes employés à travers les très vastes sujets des sciences biomédicales.

Summary of the Innovation: Des bases de données lexicales bilingues, appelées MT (mémoires de traduction), ont dû être créées pour alimenter un logiciel de traduction assistée par ordinateur. Ces MT 
ont entre autres été bâties à partir de traductions officielles des objectifs d'études provenant des organismes régulateurs de la compétence des médecins en formation au Canada soit: le CMC, le CRMCC et le CMFC. Le contenu du curriculum étant majoritairement en format PowerPoint, donc avec peu de contexte, il fut difficile d'assurer la fiabilité de la traduction automatisée. Un processus de révision à plusieurs étapes a dû être appliqué pour y remédier. En plus des traducteurs, ceci impliquait le responsable académique, les responsables de volets/de cours, les futurs professeurs, etc.

Conclusion: Le projet de francisation du curriculum jouit de plusieurs bénéfices: I'uniformisation du lexique de tous les cours; le développement d'un lexique médical anglais-français (québécois); le développement de moyens techniques et d'une expertise en matière de francisation de grands volumes de documents sur des sujets spécialisés; la mise en place de structures informatiques pour faciliter la gestion et la collaboration dans la révision des documents; un engouement des autres facultés de l'Université McGill quant à la francisation éventuelle de leurs programmes.

\section{DP 21-2 Assessing Generalism in Undergraduate Medical Education}

Melissa Nutik University of Toronto, Risa Freeman University of Toronto, Nicole Woods University of Toronto, Azadeh Moaveni University of Toronto, Ruby Alvi University of Toronto, James Owen University of Toronto, Jared Gleberzon University of Toronto

Background/Purpose: Generalism is a widely held fundamental value in medical education. In recent years, medical schools have purported to emphasize generalism in their Undergraduate curricula. How are these schools assessing their level of success in accomplishing this goal? The literature does not provide evidence of a feasible or reliable tool, or a description of a program used to assess the degree to which generalism is included in learning materials. We sought to develop and apply an evidenceinformed program to assess the degree to which generalism principles were present in our Preclerkship Undergraduate curricular learning materials and to provide recommendations to enhance generalist content.

Summary of the Innovation: An environmental scan of the literature and accreditation documents was conducted to identify key elements of generalism and develop an evidence-informed tool. The tool was applied to 61 weeks of case-based learning cases. Feedback from clinician educators was sought and incorporated into the creation of the Toronto Generalism Assessment Tool (T-GAT). Subsequently, a study was conducted to assess the feasibility and reliability of T-GAT.

Conclusion: This is the first report of an evidenceinformed program to assess the degree of generalism reflected in medical education curricular documents. The T-GAT is feasible and reliable and can be used by other medical schools wishing to review their Undergraduate curricula from a generalist lens.

DP 21-3 Evaluation of an on-line transgender health training program

Michael Lee-Poy McMaster University

Background/Purpose: TransEd is an online transgender health education curriculum developed to address the paucity of training in transgender health in healthcare professional schools. A study of six medical schools in Canada found that only two devoted more than eight hours of instruction to transgender related curriculum while the remaining allocated less than 4 hours of instruction. Selfreported comfort level showed that only $7 \%$ of students across Canadian medical schools actually felt "sufficiently knowledgeable" to assist a patient requesting hormonal therapy. TransEd is an innovative online curriculum that was developed through a grant from the Ministry of Training Colleges and Universities in 2015 to address this gap in education in medical training. This project looks at evaluating the effectiveness of an online TransEd program to address the knowledge gap, attitudes and comfort level in providing transgender healthcare.

Methods: This project used the Kirkpatrick's four levels of training framework which is considered a gold standard for evaluating training programs. A mixed-methods approach using surveys and focus groups was implemented. After completion of the 
TransEd modules, Family Medicine residents completed a survey addressing knowledge and attitudes on transgender health. In addition, a focus group with a experienced and trained facilitator conducted a small group guided session exploring TransEd's effectiveness in meeting educational needs, perceived gaps in the modules and effectiveness in increasing comfort level in addressing transgender healthcare needs.

Results: This abstract focuses on the qualitative analysis. A total of 6 Family Medicine residents participated in the focus groups. General themes that emerged including appreciating videos of true narratives that personalized the transgender journey and stories, a logical step wise approach to the modules, the practical approach to management options and the link to resources. Overall participants felt TransEd was a great foundation for transgender health knowledge and helped increase comfort levels. Suggestions for improvement included having more case examples that would model how to implement the guidelines in a practical manner and receiving ongoing training in these areas. Another limitation identified was the ability to transfer this knowledge into real life situations and the need to pair TransEd with clinical exposures and experiences.

Conclusion: TransEd is an online education program that is an effective way to deliver curriculum on transgender health. Medical residents appreciated the practical approach that helped to increase their comfort levels in providing transgender care. However, pairing this with clinical rotations and experiences would further enhance the effectiveness of TransEd. Further research is needed to study the affect of TransEd on behavioural change in medical professionals providing transgender care.

\section{DP 21-4 A concerted approach to}

\section{curriculum mapping by summer students}

Sietske Speerstra University of Alberta, Daniel Livy University of Alberta, Hollis Lai University of Alberta, Tracey Hillier University of Alberta

Background/Purpose: A medical school curriculum consists of thousands of scheduled learning events delivered by a body of instructors that is of the same order of magnitude. A comprehensive review of the curriculum is critical to its efficient and effective delivery. Curriculum overviews can be generated from several perspectives. For example, each session is associated with learning objectives, documenting the curriculum as it is planned. To gain the perspective of how the curriculum is delivered, the MD Program engages 8 to 18 students each summer to assign a set of keywords (nodes) to scheduled learning events and assessments. Together, the curriculum and assessment maps form a searchable database that can be used to analyze the coverage of a topic across the curriculum, discrepancies between planned and delivered curricula, or performance in certain topic areas.

Methods: Over the last 5 years, summer mapping students were provided with a standardized list of 580 nodes, such as LMCC objectives or CanMEDS roles. Each student was then assigned a section of the curriculum to link these nodes to. Depending on the number of mapping students employed, this approach generated 2,000 - 10,000 linkages annually. While a standardized node list ensures consistency between students, the resulting map may not describe our curriculum to its fullest extent. In addition, without processing the data, the map provided little information about the relationship between nodes (e.g. "mutation" relating to "genetics"). To overcome these limitations, we revised our mapping approach to be student-centric. Rather than assigning a series of events to be mapped, we gave them the responsibility for one or more content areas (e.g. "biochemistry" or "ethics") based on their pre-indicated interests. Students then determine a list of nodes relevant to this area and map those to any applicable event in the curriculum.

Results: Democratizing topic selection and engaging students in defining content areas expanded the list of nodes to over 4,300 and doubled the map to 20,000 linkages. In addition, the map now groups nodes together in 27 content areas increasing the connectivity between topics.

Conclusion: A simple redefinition of our approach strengthened students' commitment to the project and generated a more comprehensive map with greater connectivity between topics without increasing any of the invested resources. 


\section{DP 21-5 Pediatric Developmental Screening}

\section{Curriculum: Needs Assessment}

Cara Dosman University of Alberta, Debbi Andrews University of Alberta, Andrea Davila Cervantes University of Alberta, Qi Guo University of Alberta, Sheila Gallagher University of Alberta, Sabrina Eliason University of Alberta, Carol Hodgson University of Alberta

Background/Purpose: Learning normal child development and anticipatory guidance are objectives shared by the Royal College of Physicians and Surgeons of Canada for postgraduate pediatric training, Paediatric Undergraduate Program Directors of Canada, and Canadian Undergraduate Family Medicine Education Directors. Because developmental monitoring with standardized screening instruments is one way to accomplish this objective and there are no Canadian citations in the sparse screening curricula literature, we developed a novel curriculum for General Pediatrics (GenPeds) residents and examined availability of screening curricula nationally in Canada.

Summary of the Innovation: Surveys to all GenPeds Program Directors (PDs) and Undergraduate and Clerkship Program Directors (UDs) for pediatrics and family medicine (response rate 14/17 GenPeds PDs, $6 / 17$ pediatric and $9 / 17$ family medicine UDs) showed most residencies value teaching screening to monitor child development, provided through lectures and hands-on practice in GenPeds clinics and Developmental Pediatrics (DevPeds) rotations. Screen teaching and use are absent in pediatric clerkships. Rarely used in other programs are short answer questions (SAQ), interactive workshops, and PEDS (Parents' Evaluation of Developmental Status)+PEDS:DM (Developmental Milestones). Introduced at our site were screening practice day in GenPeds clinics during DevPeds rotations (mean volume PEDS 11, PEDS:DM 3), interactive workshops, and longitudinal SAQ formative assessments. On evaluation, screen volume was strongly correlated with SAQ scores $(r=0.452, p=0.016)$ and thinking it important to screen $(r=0.385, p=0.032)$.

Conclusion: National screening training, important in pediatric residency, could include high volume screening day with formative assessments.
DP 21-6 Améliorer la préparation à l'externat : études médicales de premier cycle (émpc), uOttawa

Victoria-Marie Cusson University of Ottawa, Gladys Bruyninx University of Ottawa, Stefan de Laplante University of Ottawa

Background/Purpose: Pour les étudiants en médecine, la transition entre le pré-externat et l'externat est une période clé des études médicales de premier cycle. Ainsi, à l'université d'Ottawa (uOttawa), il serait primordial d'effectuer régulièrement une révision du curriculum, plus particulièrement l'unité d'intégration (dernière unité du pré-externat) et le stage préparatoire (première unité de l'externat). Notre projet de recherche visait à identifier les concepts clés qui permettraient d'améliorer la préparation à l'externat.

Methods: Un mapping du curriculum médical de I'uOttawa a été effectué en le comparant à ceux des autres facultés de médecine du Canada. Par la suite, une recension structurée des écrits a été complétée afin de ressortir les concepts théoriques qui pourraient servir de fondement aux modifications du curriculum permettant une meilleure intégration des connaissances.

Results: À l'uOttawa, l'unité d'intégration est divisée en $54 \%$ de cours didactiques, $19 \%$ d'activités pratiques et $21 \%$ d'ateliers. Pour le stage préparatoire, $22 \%$ sont alloués aux cours didactiques, $29 \%$ aux ateliers et $48 \%$ à la pratique en milieu hospitalier. Pour améliorer la préparation à l'externat, des modifications bénéfiques pourraient être apportées, notamment en priorisant l'apprentissage actif ; en mettant l'intégration cognitive en premier plan en salles de classe; en introduisant les sujets les plus complexes à la fin du pré-externat selon une approche de cycle de vie ainsi qu'en associant une séance de développement des aptitudes cliniques à chaque séance d'apprentissage par cas.

Conclusion: Nous avons réussi à suggérer des recommandations pour l'amélioration de la préparation à l'externat. 
DP 21-7 The Impact of PHarmacy Students as Educators (PHASE) and a Simulated Teaching Environment in an Entry-toPractice PharmD Program at UBC

Fong Chan University of British Columbia, Tony Seet University of British Columbia, Janice Yeung University of British Columbia, Sharon Leung University of British Columbia, Kimberley MacNeil University of British Columbia, Reanne Li University of British Columbia, Justine Saran University of British Columbia, Sandra Jarvis-Selinger University of British Columbia

Background/Purpose: During the 2017-2018 academic year, the PHASE Program was piloted with Year 2 (PY2) Entry-to-Practice (E2P) PharmD students at UBC. The program allowed students to see themselves as pharmacist-educators by providing a simulated teaching environment.

Methods: During this pilot year, PY2 PharmD students participated in a didactic session on effective teaching and the following week, students were assigned to small groups where each student delivered a minilesson during an academic-half-day (AHD). Feedback was provided after each session. Quantitative survey data, using a Likert scale, was collected from students before the didactic session and after the AHD to evaluate students' beliefs about their confidence and competence in teaching. Qualitative data in the form of responses to open-ended questions were asked post-AHD to gather students' self-reflections about the simulated teaching session.

Results: Before the didactic session, $39.8 \%$ of respondents agreed or strongly agreed that they are confident in their ability to educate a group. After the AHD, this number increased to $80.7 \%$. After the AHD, the number of respondents who felt they are able to conduct an educational session increased from $42.8 \%$ to $80.3 \%$. Analysis of open-ended responses revealed insight into how PHASE impacted students' beliefs about themselves as pharmacist-educators.

Conclusion: Based on student survey data, the PHASE program had a positive impact on students' selfreport that they are able to educate a group and conduct an educational session. Next steps include implementing and evaluating the second and third years of the PHASE program.

\section{DP 21-8 Medical Council of Canada} Curricular Gaps: The development and utilization of MCC curricular gap reports

Amanda Stalwick University of Saskatchewan, Regina Taylor-Gjevre University of Saskatchewan

Background/Purpose: Striving for excellence in our curriculum to ensure we adequately cover MCC objectives is critical for medical students and universities. At the University of Saskatchewan, we have developed a process to screen for significant curricular MCC gaps in our pre-clerkship and clerkship curriculum.

Summary of the Innovation: Each of our learning objectives in our curricular management tool (One45) is mapped with an MCC objective. MCC gap reports are run for each course and results collated into an excel spread sheet with the MCC category along the top, course name on the side, and a numerical total at the bottom of each MCC category. The numerical total represents the number of individual curricular locations the topic is taught in the four-year program. This visually appealing colour coded table is easily interpretable by educational leads and other faculty members. Results: The collated report is analyzed and MCC categories that indicate fewer than 10 locations are identified and then investigated further. Key word searches and cross comparison are used to determine if the report is accurate or if content is being taught and merely insufficiently mapped. Identified potential gaps are then reviewed by the curriculum committee to determine if curricular change/supplementation is required.

Conclusion: The development and utilization of MCC gap reports has enabled us to properly identify MCC curricular gaps and/or under mapping in our curriculum. Gap reports have allowed us to undertake a review of our curricular mappings to topic areas to ensure the success of our students and verify quality assurance. Additionally, the MCC curricular gap report supports our Curriculum Committee review process to efficiently and quickly focus on areas that are deficient in our curriculum. 


\section{DP 21-9 A Longitudinal Integrative} Resilience Curriculum for Medical Students

Shayna Kulman-Lipsey University of Toronto, Joanne Leo Leo University of Toronto, Samantha Yang University of Toronto, Arshia Javidan University of Toronto, Andrea Levinson University of Toronto, Leslie Nickell University of Toronto

Background/Purpose: It is well-documented that student well-being is challenged in medical school. The Doctor of Medicine (MD) program at the University of Toronto developed a four-year resilience curriculum (RC) to encourage students to reach out for help and equip them with resiliencebuilding strategies.

Summary of the Innovation: In 2016, the first phase of the resilience curriculum was embedded into the core curriculum. The curriculum now spans all four years. The Resilience Curriculum (RC) is composed of online modules, small group workshops, and Monologues in Medicine, narratives of medical trainees sharing experiences of mental health, wellness and resilience. Recognizing that the onus of resilience is not just on the individual, faculty development to support the curriculum, as well as strategies to address systems issues have been incorporated into the curriculum.

Conclusion: Preliminary data suggests students enjoyed learning new strategies and found the skills applicable to their lives and perceived some value in all activities. Students preferred the delivery of workshops over a lecture-based format noting that there was more engagement. Students also made recommendations regarding logistics of delivery such as workshop size, length and scheduled time. Faculty, student affairs staff and students developed and implemented the curriculum. Evaluation based on feedback from implementers and developers of the curriculum has recently been completed. The overall aim of this work is to contribute to a cultural shift in medicine that values and responds to the importance of physician wellness and resilience. This can only be accomplished through a collaboration of students, faculty and the larger medical community.

\section{DP 22-1 Implementation of a Jigsaw Classroom to Teach Pharmacy Students}

Colleen Brady University of British Columbia, Michelle Fischer University of British Columbia, Tony Seet University of British Columbia

Background/Purpose: The Jigsaw teaching technique (https://www.jigsaw.org/) was implemented into Professional Years (PY) 1-3 of a 4-year entry-topractice PharmD program at the UBC. A Jigsaw classroom was used to teach HEENT dosage forms in PY1, respiratory dosage forms and diabetic devices in PY2, and contraceptives in PY3. Although this technique has been used in elementary and high schools, its use in healthcare education is limited.

Summary of the Innovation: Prior to the tutorials, students were divided into groups of 6 students. One student from each group was assigned to be the lead for a particular topic or "segment". The lead was asked to be prepared to teach their segment to their small group. At the beginning of the tutorial, all the leads for a particular segment met to solidify their knowledge and discuss teaching strategies. The student groups then rotated through six stations; during which each lead took turns teaching their segment to their group. The session concluded with a Faculty member reviewing the key points from each segment with the entire class.

Conclusion: Based on PY1 student survey data and Faculty observations, the Jigsaw technique improved the student learning experience by reinforcing their learning by teaching and working collaboratively with their peers. Suggestions for improvements include more comprehensive preparatory materials and accountability for topic leads to be prepared. Next steps include expanding the use of this technique to teach other topics, and measuring its impact on students' retention of knowledge and skills and their teaching ability. 
DP 22-2 Examining self-regulated learning behaviours of incoming PGY1 residents in two Canadian family medicine programs

Shelley Ross University of Alberta, Alexandra Aquilina University of Alberta, Rob Kiddell University of Victoria, Deena Hamza University of Alberta, Theresa van der Goes University of British Columbia, Cheryl Poth University of Alberta, Shirley Schipper University of Alberta

Background/Purpose: Healthcare professionals must engage in lifelong learning to keep up to date with the immense amount of new information generated every year. A goal of competency-based medical education (CBME) is to support learners in developing good lifelong learning skills. Self-regulated learning (SRL) is a theoretical approach to explain how learners proactively self-direct their learning behaviours and cognition in formal or informal learning contexts. Learners who engage in adaptive SRL plan their time, organize their study strategies, and are flexible in their approaches to learning as determined by their goals. In this study we measured the SRL behaviours of incoming PGY1 residents at two Canadian residency programs to determine the level of SRL skills that residents bring into training.

Methods: A survey was developed based on three existing validated instruments to measure residents' self-reported SRL skills and behaviours, including metacognitive self-regulation, critical thinking, cognitive awareness, and achievement goal orientation. Surveys were distributed at two residency programs in the first 6 weeks of the first year of residency. Ethics approval was obtained at both sites.

Results: Surveys were completed by $50 \%$ of residents at Site A and $25 \%$ of residents at Site B. Self-reported SRL skills were similar at both Sites, with the exception of mean scores on Declarative Metacognition $(A=6.17 ; B=5.53)$ and Information Management Strategies $(A=7.78 ; B=8.03)$.

Conclusion: This study provides insight into the SRL skills that learners have upon completing medical school, and gives the subject programs a baseline to allow for determination if CBME can improve SRL skills over time in residency training.
DP 22-3 Med Combat Comics - A graphic novel approach to medical education in the armed forces.

Paige Blumer University of British Columbia, Michael Claydon Royal Dragoon Guards, British Army, Katey Murray Engineer Regiment, British Army, Claudia Krebs University of British Columbia

Background/Purpose: Medical emergencies can happen anytime, anywhere, but some environments are complex and have specific needs and urgency. One of these environments is a medical emergency in the military, in particular in the field. Medics need continuous training and up to date information and we have been looking into an engaging way to convey this critical information with a focus on accuracy in the military context and a story that will stick and be memorable.

Summary of the Innovation: For this purpose we have developed a graphic novel approach to tell relatable stories of military life with a medical emergency that requires an intervention. The story has its main component of the soldier developing an illness or a situation that requires an intervention and in addition to that there is a visual side story of the physiology of the internal organs and systems that are responding to the situation. This dual approach allows us to blend the development, presentation, and treatment of a medical emergency with the underlying physiology.

Conclusion: This approach allows for the presentation of complex scenarios and how to deal with them in a military context in an engaging and comprehensive way.

\section{DP 22-4 A case-based interactive lecture on pathological sonographic findings}

Sabine Schneidewind Hannover Medical School, Philip Bintaro Hannover Medical School

Background/Purpose: Curricular practical ultrasound classes become increasingly common in medical schools. These resource-intensive small group classes rely on healthy subjects performing ultrasound on one another and therefore often do not include any pathological findings. But it is the pathological findings that many young physicians are expected to 
recognize from early on in their residency which indicates the need to address these topics in undergraduate medical education.

Methods: We conceived a lecture format addressing recognition and precise description of pathological sonographic findings. The topics of kidney cysts, liver cirrhosis as well as urinary obstruction were moulded into one case each with patient history and findings of physical examination, laboratory tests as well as abdominal ultrasound. 89 fifth-year undergraduate medical students attended this four-hour interactive case-based lecture session including 1 . live demonstration of finding and measuring liver and kidneys, 2. describing and interpreting pathological findings together as well as 3 . clinical decision making by integrating information from all available sources. Orientation and recognition of pathological findings were assessed via a short-written test before and after the lecture.

Results: After the lecture, students scored significantly higher in correctly identifying 1 . the liver (1 versus .52 out of one point) 2 . the section level in a sagittal transhepatic image of the right kidney (.9 versus .29 out of one point), 3. the sonographic criteria for cysts ( 3.26 versus .86 out of five points) as well as 4 . for liver cirrhosis ( 2.5 versus .55 out of five points) after the lecture.

Conclusion: Our results show that students' orientation and recognition regarding sonographic findings can be increased by an interactive, casebased class resembling every-day physician routines. During evaluation students emphasized that particularly the integration of pathophysiological knowledge into the process of clinical reasoning was helpful. We plan assessing long-term retention of acquired skills after three months. Take-home message: Recognition of pathological findings can be taught effectively in a resource-sparing lecture format. Regularly and repeatedly including the pathophysiological background in clinical cases may contribute relevantly to students' understanding of the underlying disease.

\section{DP 22-5 FAST EVIDENCE: Building a toolkit to optimize patient care}

Hadas Haft University of British Columbia, Sarah Tajani University of British Columbia, Bob Bluman University of British Columbia, Karen MacDonell College of Physician and Surgeons of BC, Brenna Lynn University of British Columbia

Background/Purpose: Within a complex healthcare environment and a rapidly evolving digital world, physicians need to be adept at identifying credible, evidence-based information online. To help meet that need, the College of Physician and Surgeons of $B C$ (CPSBC) in collaboration with UBC Division of Continuing Professional Development (UBC CPD) developed the Finding Medical Evidence course in 2007. The course introduced Point of Care (PoC) online tools to optimize patient care. Recently, the curriculum, format, and branding was redeveloped to integrate best practices in CPD and adult learning principles.

Summary of the Innovation: FAST Evidence is an interactive, small group workshop facilitated by the CPSBC librarians. Using real-life case examples, participants gain hands-on experience in developing effective internet searches to find credible and evidence-based medical information. At the end of the workshop, learners complete a Commitment to Change form to plan for practice improvement. To further support ongoing learning, participants engage in incremental post-program activities including a peer check-in and librarian coaching session. The post-workshop coaching session gives learners the opportunity to receive objective feedback and support from program facilitators. Evaluation data is collected directly onsite and at 12 weeks postworkshop.

Conclusion: The onsite evaluation data indicates a $43 \%$ increase in participants' reported comfort level using PoC tools. Further, quantitative and qualitative data from the 12-week reflective activity demonstrates an increased level in confidence and satisfaction using the tools in practice. Overall, data collected suggests that this innovative program is effective in teaching physicians how to effectively navigate and find credible, evidence-based information at the PoC. 
DP 22-6 BCPoCUS.ca: A Clinically-Oriented Ultrasound Education Website Interface

Kathryn Young University of British Columbia, Bianca Boicu University of British Columbia, Oron Frenkel St. Paul's Hospital

Background/Purpose: Point-of-care ultrasound (PoCUS) is a bedside procedure typically performed in the emergency room to inform practitioners' clinical assessment and decision making. As PoCUS technology becomes increasingly available and integrated into rural practice, it is important that medical education resources grow and evolve with the changing landscape. By developing readily accessible education organized in a clinically-oriented interface, practitioners are better supported to care for patients at the point-of-care.

Summary of the Innovation: Through collaboration with health care providers who seek to safely expand PoCUS practice across $\mathrm{BC}$ and user testing with the target audience, BCPoCUS.ca was designed to serve as a free, open access learning tool and educational database offering carefully curated PoCUS content. Content is grouped into three main thematic categories: (1) presenting problems, (2) organ systems, and (3) procedures, and an interactive home page with an optimized search feature enables visitors to efficiently navigate to their area of interest. Practitioners may consult rapid summaries during time-limited visits, or further their learning through videos and how-to guides outside of the clinical setting.

Conclusion: As an educational tool, BCPoCUS.ca provides resources to simplify the challenging process of integrating PoCUS into clinical practice. Over 2,466 users from 81 countries have consulted the website since its launch on September 26, 2018. Through further user testing and consultation with medical practitioners during future development phases, the BCPoCUS.ca project will continually explore how engagement with a target audience can inform the development of an accessible, efficient, and timely tool that meets learner and user needs.
DP 22-7 Experiential Nursing Rounds: An opportunity for graduate nurses to process challenges, develop coping strategies, and experience social support within the academic curriculum

Camila Velez McGill, Nicole-Ann Shery McGill, Deborah Friedman McGill

Background/Purpose: Nurse trainees experience a variety of stressors in academic and clinical environments, including heavy workloads, challenging patient interactions, and conflict between staff. Occupational stress may lead to burnout, powerlessness, isolation, and attrition from nursing. It is imperative to provide trainees with coping resources to deal with stressors, prevent negative outcomes, and foster emotional well-being. Social support, within the context of support groups, has been found to have a beneficial effect on health outcomes within the nursing population.

Summary of the Innovation: The WELL Office in the Faculty of Medicine at McGill University developed the Experiential Rounds, two confidential support groups for nurse trainees ( $N=20 /$ each), which are offered as part of the academic curriculum. The Experiential Rounds are designed and facilitated by a Wellness Consultant and Registered Psychotherapist, with the goal of helping trainees share their clinical experiences, develop strategies to overcome hardship, build confidence and community. Evaluation results revealed that most students found the sessions useful and helpful to their academic and clinical journeys. Thematic analysis further revealed that students were able to: a) normalize challenges experienced, b) learn practical tools to manage stress, c) alleviate perfectionism, and d) share experiences in a confidential and safe venue. Suggestions included to have more sessions with smaller student groups.

Conclusion: Experiential rounds may be a promising tool to enhance nurse trainees' ability to cope with stress. Embedding experiential rounds within the curriculum can validate the importance of emotional well-being for a healthier nursing workforce. Recommendations and feedback for implementation of sessions will be shared. 
DP 22-8 Assessing mistreatment among medical students at different stages of undergraduate education

Richard Pittini University of Toronto, Yuxin Tu University of Toronto, Lisa Robinson University of Toronto, Glenys Babcock University of Toronto, Mariela Ruetalo University of Toronto

Background/Purpose: Mistreatment (discrimination \& harassment) is a concerning issue in Canadian Medical Schools. This study aims to identify how mistreatment changes when students transition from preclinical into clinical education.

Methods: We surveyed preclinical students in 2018 and all years in 2019. Students were asked if they had personally experienced mistreatment. Data was available for 188 participants. Generalized Estimating Equations were used to examine how mistreatment changed between the two years and how it differed between two cohorts after adjusting for site of training, age, gender and ethnicity.

Results: The proportion of the class of $2 \mathrm{~T} 1$ reporting discrimination during clinical education in 2019 was $52.6 \%$ compared to $38.5 \%$ during preclinical education in 2018. The proportion reporting harassment was $32.1 \%$ vs. $17.9 \%$ (clinical vs. preclinical). The proportion of the class of $2 \mathrm{~T} 2$ reporting mistreatment did not change between two preclinical years. When comparing the results between two years, the models indicate that the change for 2T1 in experiencing discrimination from 2018 to 2019 is greater than that for $2 T 2$ (OR=1.9 vs. 1.4). The degree of cohort difference in year-to-year change in harassment is more substantial (2T1 $\mathrm{OR}=2.1$ vs $2 \mathrm{~T} 2 \mathrm{OR}=0.7)$. Differences between cohorts in mistreatment were noticeable in 2019 (OR=2.8 and 2.8 discrimination and harassment) but this pattern is not observed in 2018 (OR=1.9 and 1.2).

Conclusion: This study provides evidence that mistreatment affects a significant proportion of medical students and although there is some variation between classes, the significant rise in incidence appears to be related to the transition from preclinical to clinical education.
DP 23-1 Project-Managing the Poetry of Medicine: Structure and Meaning in an Accreditation and Quality Improvement Unit

\section{Fernanda Claudio McGill, Patricia Wade McGill}

Background/Purpose: Competence-Based Medical Education (CBME) poses new challenges for accreditation and quality improvement of residency programs. Medical education is a complex enterprise, engaging such multi-faceted dimensions such as professional identity, and the need to balance personalized care that meets requirements of health systems. While CBME appears straightforward and self-explanatory, the formal model is challenged by context of implementation (system) and social reproduction of medicine (meaning). Lack of coordinated models of how medical educators could work to satisfy such complex and diverse requirements challenges the philosophy of CBME.

Summary of the Innovation: To offer a potential model, the Office of Accreditation and Education Quality Improvement (OAEQI) adopted a team-based approach to support medical training in the context of diverse populations, the Quebec health system, medical education best-practice, and national accreditation standards. OAEQI functions through a tripartite service model that aligns curriculum, assessment and evaluation delivered through the lens of quality improvement and accountability in its various institutional and social dimensions. Our strength is our team. We are bilingual and demographically varied working collaboratively and deriving our expertise from psychology, medical anthropology, nursing, political science, education, medicine, and others. Our structure is enabling and humanistic in terms of work practice and deliverables. We illustrate this approach with a case study of a residency program.

Conclusion: Our multidisciplinary approach enables identification of complex educational needs, barriers, and opportunities, providing an exemplar of TeamBased Quality Improvement. Our response model addresses residency program needs accounting for strengths and constraints, measures of outcomes and impacts, and feedback. 


\section{DP 23-2 Effective speakers in Continuing Medical Education}

Diana C Sanchez-Ramirez University of Manitoba, Christine Polimeni University of Manitoba

Background/Purpose: Studies exploring speakers' effectiveness in Continuing Medical Education (CME) are lacking. This study sought to identify relevant presentation characteristics associated with speaker effectiveness in the context of CME.

Methods: This study used data on speakers' evaluations from $26 \mathrm{CME}$ presentations in various topics, which took place on five different days (between 4 to 6 lectures per day) in the academic year 2018-2019 at the University of Manitoba. Data was collected using an audience interaction tool for meetings (sli.do). Overall effectiveness of the speaker was rated using a 5-point Likert scale. Additional presentation characteristics evaluated (4-point Likert scale) were: a) the information was clearly presented, b) the presentation was balanced, c) the presentation was consistent with the stated objectives, and d) the presentation provided information which will influence my practice. Responses of speaker effectiveness were dichotomized (no/yes) to avoid celling effect. Statistical analyses were completed using logistic regression with Generalized Estimated Equations (GEE) clustered at the lecture level. Crude models explored at the association of speaker effectiveness with each characteristic at the time, and an adjusted model incorporated the four characteristics at the same time.

Results: Nine hundred and ten speakers' evaluations were submitted from 266 anonymous participants, who provided evaluation for a mean of three lectures/day ( $\min 1, \max 6)$. Ninety-one percent of the responses rated the speakers as effective. Crude models indicated that effective speakers had statically significant higher scores in the four characteristics assessed (range between 2.5 and 3.3 points) than the non-effective speakers. However, the adjusted model indicated that effective speakers received higher rates only in the following items: a) the information was clearly presented $(B=1.6,95 \% \mathrm{Cl}$ 0.9- 2.3; $p<0.001)$, b) the presentation was consistent with the stated objectives $(B=1.2,95 \% \mathrm{Cl}$ 0.5-1.9; $p=0.001)$, and the presentation provided information which will influence my practice $(B=1.9,95 \% \mathrm{Cl} 1.3$, $2.5 ; p<0.001)$. A balanced presentation was no longer associated with speakers' effectiveness in the adjusted model ( $\mathrm{B}=0.1,95 \% \mathrm{Cl}-0.7,0.7 ; \mathrm{p}=0.94)$.

Conclusion: Results suggested that speakers perceived as effective incorporated in their CME lectures information meaningful for the participants and consistent with the stated objectives, and presented clearly. This information would inform speakers' improvement activities.

\section{DP 23-3 Are We Ready For What's Coming? Scholarship, Leadership and Continuing Professional Development}

Morag Paton University of Toronto, Paula Rowland University of Toronto, Walter Tavares University of Toronto, Suzan Schneeweiss University of Toronto, Shiphra Ginsburg University of Toronto

Background/Purpose: Effective Continuing Professional Development (CPD) is needed to ensure a competent and sustainable health system. As new competency-based models of CPD come on the horizon, CPD scholarship is required to understand and optimize their impact, yet scholarship may appear undervalued. Our research question was what are the scholarly profiles, aims and needs of CPD program/conference directors?

Methods: We developed, piloted and distributed a 35-item survey to 400 Program and Conference Chairs. Narrative responses were coded and thematically analyzed; quantitative data were analyzed using descriptive statistics.

Results: We had a $20 \%$ response rate to our survey. Results indicate that most program/conference directors did not need to formally apply for their roles, but volunteered (37\%) or were appointed (40\%). Academic Ranks are distributed - 32\% are Professors, 25\% are Associate Professors, 32\% are Assistant Professors. 62\% have some training in education. $45 \%$ of respondents plan on conducting scholarship although $50 \%$ state that they do not have access to scholarly support despite the presence of research support/services. Lack of time, funding and the undervaluing of scholarship are listed as barriers to pursuing scholarly work. 
Conclusion: These findings build on an earlier interview-based phase of this study. CPD educators and leaders move into their roles in an unplanned way and face challenges in approaching their work as scholarship. In our large distributed network of CPD leads, existing scholarship supports need to be better publicized, with more advocacy work needed to ensure that CPD scholarship is recognized and valued throughout the system.

\section{DP 23-4 The changing landscape of Continuing Professional Development/Continuing Medical Education (CPD/CME) in Canada: A scoping review.}

Francesca Luconi McGill, Elizabeth Wooster University of Toronto, Morag Paton University of Toronto, Meron Teferra McGill, Andrea Quaiattini McGill, Suzan Schneeweiss University of Toronto

Background/Purpose: In North America, despite the urgent call in 2003 to implement a significant health care reform, change has been slow or non-existent. Continuing professional development (CPD) aligned with the healthcare system and the evolving patients' needs is proposed as an agent of change to support this paradigm shift. Research Questions: What forces have influenced physicians' CPD in Canada over the last decade? What type of terminology, conceptual frameworks and methods are reported in the literature?

Methods: Guided by a 6-stage framework, this scoping review covered published literature following these inclusion criteria: physicians, in Canada, available in English/French between 2008-2019. Five bibliographic databases were searched and datacharting was developed based on an iterative process. Studies were coded independently and reviewed in pairs to ensure accuracy and reliability.

Results: Of the original 3761 references, 532 articles met our inclusion criteria. Two thirds targeted physicians alone. Sixteen percent targeted interprofessional teams, mainly composed of physicians, nurses and residents. Seventy-three percent of articles focused on continuing education techniques and approaches and 63\% explicitly discussed clinical and non-clinical content areas.
Workshops (15\%) and case-based instruction (12\%) were the most commonly used instructional methods. Of the articles reviewed, the terms "CME" and "CPD" appeared in 34\% and 26\% respectively and $11 \%$ cited a theoretical framework.

Conclusion: This scoping review will contribute to the evolution of the CPD field by: keeping physicians upto-date as clinical practitioners, teachers and scholars; informing CPD developers, licensing, regulatory bodies and policymakers, and identifying gaps for future research.

\section{DP 23-5 Auditing Rounds in a Distributed Medical Education Environment: An Innovative Approach}

Clare Cook Northern Ontario School of Medicine, Lisa Kokanie Northern Ontario School of Medicine, Julie Colquhoun Northern Ontario School of Medicine,

Background/Purpose: The NOSM CEPD Office certifies and accredits a number of regularly scheduled series, including rounds, educational series, and journal clubs. In keeping with NOSM's distributed model rounds are organized by local faculty communities of practice, leading to the challenge of finding an efficient and effective way to audit these rounds for compliance with CFPC and RCPSC standards, while also maintaining a supportive, collaborative relationship with our faculty-led Scientific Planning Committees (SPCs).

Summary of the Innovation: The office developed a short online survey requesting uploaded documentation across accreditation requirements, including promotional materials, conflict of interest, presentation content, identification and mitigation of potential bias, and record keeping. A review and escalation protocol mirroring the approval process allows any compliance issues to be identified and addressed.

Conclusion: The most common compliance problems have been in ensuring all of the appropriate information is included in the promotional materials, and in ensuring complete record-keeping. Those filling out the audit survey found it was short and easy to comply. One noted afterwards that as soon as she looked at it, she knew what she needed to fix for next time. Making SPCs aware of the audit process ahead 
of time has helped to focus it on improvement and education, particularly for committees who are new to the process and requirements. Universal audits also help to mitigate the risk that non-compliance issues slip through the cracks. The online survey format allows us to track data over time and across programs with minimal additional work.

\section{DP 23-6 A continuous quality improvement model for person-centered clinical education.}

Susan Cauti University of Alberta, Heather Gaunt University of Alberta, Kari Osmar University of Alberta

Background/Purpose: An undergraduate radiation therapy program expanded to include provincial clinical practicum sites. Diverse procedures and technologies required deliberate mechanisms to support student learning needs. A continuous quality improvement (CQI) process was developed to assure ongoing quality, currency, and relevance of the curriculum to learners. CQI curriculum processes are ubiquitous. The purpose of this version - innovative to us - was to support equitable person-centered learning across a provincial education program, in a timely and nimble manner. Student growth was a reciprocal outcome of faculty and program evolution purposefully addressed via structured CQI.

Summary of the Innovation: Weekly CQI was integrated into all clinical courses and involved Clinical Faculty (CF) at each practicum site and the Teaching \& Learning Specialist. The collaborative process was ongoing and iterative; it was considered a standard component of the work done by CF. Adaptations and ideas were captured weekly, culminating in an annual review of all course competencies, teaching strategies, and evaluations. The changes were consistently infused into clinical course design, to accommodate rapid changes occurring in the radiation therapy environment.

Conclusion: CQI reduced heuristic reactions to local pressures by providing a mechanism of checks and balances so CF made informed, reasoned decisions that were sustainable across clinical practicum sites. Faculty were challenged to become more holistic in course design and implementation, clinical partnerships, and student-centered teaching. It resulted in teaching tools and techniques that are now being expanded to didactic courses. Students reported positive perceptions of faculty support in practical and emotional aspects of clinical learning.

\section{DP 23-7 The use of theory in quality} improvement and patient safety education: a scoping review

Joanne Goldman University of Toronto, Brian Wong University of Toronto, Lisha Lo University of Toronto, Ayelet Kuper University of Toronto, Anne Smeraglio Portland Veterans Hospital and Oregon Health \& Science University

Background/Purpose: Systematic reviews of quality improvement and patient safety (QI/PS) education provide descriptions of curricular content and teaching methods, but have not explicitly described how theory underpins QI/PS education. Using theory allows a more reflective rationale and deliberate approach to planning and delivering educational programs. We therefore undertook a scoping review to examine the extent, range and nature of the use of theory in QI/PS education.

Methods: We identified articles describing QI/PS curricula targeting healthcare professionals in training and/or in practice that used theory to inform its planning, delivery, or evaluation using a systematic approach for search strategy and article review. Two team members independently extracted data from included articles. We synthesized data to describe the type and range of theories used, and how they were used to inform QI/PS education.

Results: We identified 24 articles, mostly published between 2009 and 2017. Articles varied widely in terms of target professional group (e.g., physicians, nursing, pharmacy, etc.), stage of learning (undergraduate, postgraduate and continuing education) and journal type (e.g., education, QI/PS, health services). Studies drew upon a range of learning theories, including cognitivist, sociocultural, and transformative theories, as well as theories from psychology, sociology and philosophy. Theory was used to inform the planning and evaluation of QI/PS education programs. 
Conclusion: The breadth of theories identified provides insight into the range of lenses that are potentially relevant for planning and evaluating QI/PS education. Future directions include strengthening the rigour of the use of theory and extending the repertoire of theories used.

\section{DP 23-8 Rural Medicine eMentoring BC (RMeMBC)}

\section{Takaia Larsen Selkirk College}

Background/Purpose: The RMeMBC program connects Rural Pre-Medicine Program (RPM) students at Selkirk College with medical students in the Rural Medicine Interest Group (RMIG) at UBC. Due to geographic barriers, it is difficult for rural students to find "near peer" mentors. Likewise, medical students interested in rural practice are often studying in urban settings and feel disconnected from rural communities. The RMeMBC program provides a platform where these students can form mentoring relationships online.

Summary of the Innovation: In collaboration, RPM, RMIG and the Rural Education Action Plan (REAP) developed a curriculum of eight modules highlighting the transition from undergraduate studies to medical education training. Once mentoring matches have been made based on common interests and experiences, students access the curriculum through an eMentoring software platform, and it helps to guide their conversations.

Conclusion: Early results from the program indicate that mentors and mentees find the experience valuable. Interactions on the platform are generally focussed on the topics students identified as being important to them: the transition to medical education training and the needs of rural communities. One challenge which has presented itself is that portions of the curriculum are sometimes left unaddressed. Currently, we are exploring ways to encourage greater engagement with the curriculum while still allowing for organic conversations driven by participants' interests. Upon review, all partners view RMeMBC as successfully providing a resource that supports participants with rural backgrounds or affinities, and it could be replicated to support rural students across Canada.

\section{DP 24-1 Class Years Have Reputations As Hawks or Doves, But Are They?}

Kevin Busche University of Calgary, Mike Paget University of Calgary, Wayne Woloschuk University of Calgary

Background/Purpose: Each class year in our program quickly develops a reputation as 'hawks' (tending to evaluate learning events harshly) or 'doves' (leniently) based on anecdotal perception. This profile gets propagated throughout the faculty and staff. Is there any quantitative proof?

Methods: Students at the Cumming School of Medicine are invited to complete a daily evaluation for each of their structured learning events. Over the last five graduating classes, we have evaluated over 30,000 events. Aggregating the responses of the students across years has provided a rich dataset to query. Students from the classes of 2016, 2017, 2018, 2019 and 2020 at the Cumming School of Medicine have evaluated 4442 of their lectures individually with the following question "Overall Rating" on a scale of 1. Unacceptable, 2. Below Expectations / Room for Improvement, 3. Good, 4. Very Good, 5. Outstanding. They complete these ratings within one week of the learning event.

Results: Across those separate class years, the average lecture ratings are 3.91, 3.90, 3.87, 3.90 and 3.95 respectively. The standard deviation for that data by year is: $0.34,0.43,0.39,0.39,0.44,0.46,0.38$, 0.47

Conclusion: We found that over the course of the five graduating years that their evaluations varied minimally in aggregate and were more similar than the classes' reputations would have indicated. This suggests that in situations where educational research requires a comparison of student evaluations across classes that inter-class variability is not a significant contributor to any noted differences. 
DP 24-2 Non-Critical Care Residents Exposure to Clinical and Educational Activities During ICU Rotations: A multicentered, observational study in 3 Academic ICUs

Dominique Piquette University of Toronto, Briseida Mema University of Toronto, Christie Lee University of Toronto, Alberto Goffi University of Toronto, Catharine Walsh University of Toronto, Ryan Brydges University of Toronto

Background/Purpose: In Canada, many non-critical care residency programs include mandatory intensive care unit (ICU) rotations. During ICU rotations, specialty residents are expected to develop key clinical abilities required for the care of their sickest of patients. Yet, little is known about the learning opportunities encountered by residents during typical ICU rotations.

Methods: We conducted a multicentered, prospective observational study in 3 mixed ICUs in Toronto, Canada, where non-critical care residents are supervised by critical care senior trainees and faculty. Up to four times during their first ICU month, consenting residents completed a diary survey that documented their participation in clinical (handover, rounds, procedures, admissions, resuscitations, family meetings) and educational activities (formal and informal teaching, self-study) over the previous 24h. Surveys were administered on a random day of the week (Monday to Friday) in order to sample different days.

Results: Fifty-seven residents completed 153 surveys (median: 3 surveys/resident) that described 63 different ICU days. Whereas most surveyed ICU days offered an opportunity to participate in handover (90\%), multidisciplinary rounds (73\%), and formal teaching (73\%), other activities were less frequently encountered (procedures: 46\%, informal teaching: $44 \%$; resuscitation: $35 \%$ ) or rarely encountered (family meeting: 14\%). Residents reported being the "main physician" (vs. assistant or observer) for $74 \%$ of all clinical activities in which they participated. A critical care trainee was the primary supervisor for $70 \%$ of clinical activities, and supervision was direct, indirect, or distant in $44 \%, 46 \%$ and $0.5 \%$ of the activities respectively. However, resident role and supervision varied significantly across sites.

Conclusion: Better data on resident exposure and participation in valuable clinical and educational activities are important to determine what can be realistically learned and assessed during an ICU rotation as resident experiences vary greatly across sites and over time.

DP 24-3 The clinical teaching practicum: an important learning methodology for instructional development

Debaroti Mullick Borschel University of Tennnessee, Umberin Najeeb University of Toronto, Danny Panisko University of Toronto

Background/Purpose: Clinical Teaching Practicum [CTP] is a useful teaching instrument in a longitudinal faculty development program. Each participant is required to present 20-minutes of their clinical teaching, followed by 20-minutes of class discussion and feedback, on 3 different occasions over the 2year duration of our program. Each presentation must demonstrate a different teaching modality (large group, seminar, "chalk talk", bedside, simulation/procedure, or one-on-one teaching). Presenters shared teaching sessions that either "worked well" or areas where they encountered difficulty. Other program participants provide feedback and useful insights to problem solve with their colleague.

Summary of the Innovation: CTP presentations in our program were observed and catalogued for content topics, teaching methodologies, innovation instances, nature/content of feedback, and participant satisfaction over 2-years ( $n=39$ participants). A variety of content topics and teaching methodologies were encountered. Feedback was provided on visual presentation, choice of teaching/learning style, interactivity techniques, and teaching methodology. The sessions accrued a high degree of participant satisfaction. Participants highly valued the opportunity to experiment with new teaching methods, the teaching feedback they received and the self-reflection that it stimulated.

Conclusion: CTP provides a high degree of classical face validity to an instructional development 
program. They illustrate the triumphs and dilemmas of teaching in the moment and facilitate class participants bonding and cohesion, as the group works together toward teaching solutions. CTP sessions provided a wide spectrum of teaching styles along with opportunities for teaching analysis and peer coaching. CTP can be strongly considered as a valuable component of an instructional development program.

\section{DP 24-4 University of Toronto (U. of T.)}

Postgraduate Medical Education:

Competency-Based Medical Education
Faculty Development Survey

Giovanna Sirianni University of Toronto, Susan Glover-Takahashi University of Toronto

Background/Purpose: As postgraduate programs transition to an outcomes-based approach to curriculum, assessment and evaluation, there is evidence that faculty feel unprepared for the tasks necessary in a CBME context. This project aimed to harness the collective expertise and opinion of education leaders at $U$. of $T$. to determine their experience and needs with regards to FD in CBME.

Methods: An online survey was distributed to program directors, faculty development leads and education leaders within programs that had transitioned or would be transitioning to CBME. Surveys were sent to 168 faculty members.

Results: Overall survey response rate was $44.6 \%$. Family Medicine faculty made-up $46.7 \%$ of respondents, followed by Medicine at $18.3 \%$. 56.7\% of respondents were Program Directors and $26.7 \%$ were FD leads. $80 \%$ of respondents had programs that had already launched to CBME. The top FD topics survey respondents would like to learn more about include: 1) Implementing CBME 2) Assessment tools 3) Feedback and Coaching 4) Competence Committees. The majority of programs felt that the ideal timing for CBME implementation was 6-12 months prior to implementation. The main perceived barrier to FD was lack of time amongst faculty.

Conclusion: This survey is the first of its kind to evaluate the FD needs and experiences of multiple postgraduate programs in a CBME context. Its results are relevant to postgraduate programs nationally.

DP 24-5 PANPro: a comprehensive one-year Faculty development program for new teachers

Lise Cusson Université de Montréal, Suzanne Laurin Université de Montréal

Background/Purpose: As medical education evolved and a competency-based approach was adopted in clinical teaching, our faculty development had to adapt. To meet the different stakeholders' expectations towards primary care and clinical teaching, a comprehensive faculty development program was devised aiming at better integrating new teachers into their teams, promoting a deeper understanding and mastering of teaching skills and, mostly, developing the teacher's professional identity.

Summary of the Innovation: The program: The oneyear program used educational strategies allowing the progressive building and integration of teaching competencies: learning, experiencing and reflecting. Workshops were designed to help learn the medical education concepts and experiment them in a "safe environment" with role-playing. Participants were asked to put into practice the newly learned teaching/supervision techniques and concepts in their work setting and to discuss their experiences with a local senior colleague acting as a coach. Participants were also invited to share their favorable teaching experiences or difficulties in group discussions. Their examples were then used to further develop previously learned concepts and skills or to address new ones.

Conclusion: The new teachers' faculty development program seems to have met the objectives set at the time of its creation. The health professionals ( 5 cohorts of 20-25 participants) who have completed it expressed their satisfaction at having been able to build a feeling of competence and a sense of belonging to a teacher community during the year. 
DP 24-6 Which components of the INTAPT Faculty Development course do learners identify as impactful to their learning

Judith Peranson University of Toronto, Helen Batty University of Toronto, Ancy Jacob University of Toronto, Abbas Ghavam-Rassoul University of Toronto

Background/Purpose: The INTAPT course is a foundation course for several Health Professions Teacher Education Programs (certificate, fellowship and Master's). Participants report global satisfaction and positive learning and professional outcomes. The course uses a variety of well-established pedagogical approaches to achieve learning. We sought to identify the most significant content and process components of the course that influence learning from the perspective of participants.

Methods: After a preliminary pilot we asked participants to provide a brief narrative reflection about the key element of the course that had the greatest impact on their personal and professional development. In this phase we report data from a sample of 52 out of a total 107 program participants (recruited from the 2014/15 (by email) \& 2015/16, 2017/18 (in person) classes) Reflections were analyzed using a descriptive qualitative method to identify common themes.

Results: A variety of course processes (e.g. cultivating relationships with peers, balancing challenge and support, encouraging critical thinking) and course content components (e.g. application of education theory, presenting a workshop, acquiring a toolbox of practical skills) were identified as helpful to learning.

Conclusion: In this analysis of our larger data set, we identified both content and process elements of the course that participants found impactful. Participants identified a wide range of factors. Faculty developers should utilize a variety of activities that challenge learners and at the same time support them to succeed. During this interactive poster participants will be able to place stickers next to quotes that capture their experiences in faculty development programs.

\section{DP 24-7 Faculty development to support curriculum renewal: An aligned approach}

Jana Lazor University of Toronto, Jennifer Bell University of Toronto, Lori Innes University of Toronto

Background/Purpose: Our challenge was to create a faculty development (FD) program that aligns faculty capacities and skills with goals and pedagogy of a new undergraduate medical curriculum that was undergoing significant innovation and change.

Summary of the Innovation: To ensure alignment, an eight step situational analysis was undertaken to understand: (1) proposed curriculum changes; (2) faculty roles/tasks; (3) core knowledge, skills and attitudes required to perform the tasks; (4) faculty characteristics; (5) student learning needs/characteristics that may need to considered; (6) health care environment context in which the students will be learning and faculty will be teaching; (7) educational evidence used to inform curriculum renewal; and (8) potential impact on faculty and that may need to be managed. Using this approach, FD team designed a tailored strategy for each of 12 teacher roles. Tailored tools and resources as well as multi-modal learning design were adopted for each role. During the first 2 years of the new curriculum FD offerings included 82 workshops located close to faculty clinical practice, 4 webinars, 17 educational videos, 23 print materials, 5 e-learning modules, 186 Educafe Cafes that offer coaching in local communities of practice, and peer coaching. Evaluation and scholarship are embedded in the program development process.

Conclusion: FD should be iterative and responsive to ongoing curriculum changes and newly identified faculty needs. Evaluation is key, specifically different FD modalities need to be evaluated to examine what can work, when, and why. Intentional alignment between FD and curriculum development can create a continuous quality improvement loop between the two practices that inform ongoing refinement of the curriculum $i$ as well as the faculty resources and FD learning events. 


\section{DP 25-1 The Early Introduction of Ultrasound in Undergraduate Medicine}

Eleanor Good University of British Columbia, Pete Tonseth University of British Columbia, Anthony Sturgeon University of British Columbia, Angelina Marinkovic University of British Columbia, Alasdair Nazerali-Maitland University of British Columbia, Samantha Stasiuk University of British Columbia

Background/Purpose: At UBC, we have created 3 innovations in our ultrasound curriculum over the last two years to deal with challenges in uptake, faculty recruitment and independent image acquisition.

Summary of the Innovation: The ultrasound curriculum at UBC includes introductory abdominal, echocardiography and volume assessment. Student and faculty feedback was extremely useful in finetuning these sessions. With the desire to innovate, three additions were implemented: 1) faculty development session that was run at each of our DME sites. This consisted of an expert-facilitated session with an opportunity to provide an ultrasound curriculum refresher for faculty as well as ongoing mentorship. Recruitment for the next semester's teaching sessions was complete in record time. 2) a desire to increase the overall number of scanning opportunities from 3 to 7 . Three demonstrations took place during the first weeks of term as ultrasound was modelled within the IPPA (inspection, percussion, palpation, auscultation) as an extension of the physical examination. This centred around a focussed ultrasound examination of the relevant system. Students were able to answer if their patient's dyspnoea was due to a pleural effusion, hypotension was due to a pericardial effusion or if their distension was due to ascites. 3) an online ultrasound log was created where students acquire 8 essential images including heart chambers/valves, neck and peripheral veins/arteries and views of the liver/spleen/kidneys.

Conclusion: Students now have 7 different opportunities to scan during their preclinical years and can start acquiring images independently. Tutor recruitment is complete months in advance with high quality tutors. These interventions will better prepare our students to scan in clerkship and residency.
DP 25-2 Using Outcome Harvesting to Assess CBME Implementation at Queen's University

Jennifer Railer Queen's University, Denise Stockley Queen's University, Leslie Flynn Queen's University, Amber Hastings-Truelove Queen's University, Alicia Hussain Queen's University

Background/Purpose: In 2015, Queen's University embarked on an institution-wide transition to a competency-based medical education curriculum for all 29 postgraduate medical education programs. On July 1st, 2017 this goal was accomplished. With this extensive transition came the requirement to assess the efficacy of implementation through a program evaluation process which included the use of Outcome Harvesting (Wilson-Grau). Outcome Harvesting identified the intended and unintended outcomes of CBME implementation which helped us understand how the intervention was achieved, and how the relationship between behaviours and stakeholders contributed to the successful transition.

Methods: A systematic approach to document analysis was used to categorize the 8 identified areas of implementation. Documents $(\mathrm{N}=443)$ were organized per project area, and then coded thematically. Documents were then categorized for attribution to outcomes using the Outcome Harvesting approach. Outcomes were validated via inter-rater reliability and substantiated by stakeholders to verify accuracy of formulation and plausibility of influence on the outcome.

Results: The harvest produced 38 outcomes, either intended or unintended, that can be attributed to CBME implementation at Queen's University.

Conclusion: Using Outcome Harvesting to assess the efficacy of CBME implementation produced a robust set of themes and resultant outcomes that can be categorized as requirements for success of implementation of any curricular innovation. Themes included collaboration, community of practice and stakeholder commitment. More unique observations noted through the harvest process included new policy development, creation of learner ownership and an increase in the output of scholarly activity involving $\mathrm{CBME}$ 


\section{DP 25-3 Un service de consultation} pédagogique pour les cliniciens enseignants

Suzanne Laurin Université de Montréal

Background/Purpose: Bien qu'ayant reçu une formation pédagogique, les équipes de cliniciens enseignants se sentent souvent démunies lorsqu'elles sont confrontées à des résidents en difficulté.

Summary of the Innovation: Nous avons créé un service de consultation pédagogique (appelé SAMU) pour soutenir les cliniciens enseignants dans leur démarche de diagnostic pédagogique et d'élaboration d'un plan d'intervention. Fondées sur un modèle de supervision pédagogique semblable à la supervision clinique par discussion de cas, les interventions du SAMU visent à soutenir les compétences pédagogiques des cliniciens enseignants en les aidant à trouver des solutions adaptées à leur contexte. Les équipes d'enseignants s'investissent souvent beaucoup auprès des résidents en difficulté, au risque de s'épuiser. Dans ce contexte, le SAMU aide les enseignants à mieux définir leurs attentes et limites et à accepter que leurs interventions ne donnent pas toujours les résultats escomptés. Le principal impact de ce service est le sentiment des cliniciens d'être reconnus dans leur rôle d'enseignant et dans leurs efforts pour faire face aux difficultés des résidents. II permet aussi aux enseignants, en formulant le problème, de dépasser la réaction émotive qu'il suscite et d'avoir un regard global sur la situation.

Conclusion: Les cliniciens enseignants ont parfois besoin d'obtenir l'avis de pairs expérimentés lorsqu'ils sont confrontés à des situations pédagogiques complexes. Le modèle de la supervision pédagogique permet aux enseignants non seulement de discuter de diagnostics et de plans pédagogiques, mais aussi des enjeux relationnels et contextuels spécifiques qui influent sur leur capacité à intervenir auprès d'un résident en difficulté.

\section{DP 25-4 General Internal Medicine Fellows as Teachers in the Ambulatory Clinic}

Cary Cuncic University of British Columbia, James Tessaro University of British Columbia, Harp Nagi University of British Columbia, Allison Nakanishi University of British Columbia, Aman Nijjar University of British Columbia

Background/Purpose: Recruitment and retention of preceptors in medical student ambulatory clinic is challenging; reasons cited include hospital structures being more familiar and a lack of remuneration. We hypothesized that adding General Internal Medicine (GIM) fellows as teachers in internal medicine clinic would offer benefits.

Methods: We assigned medical students to GIM fellows' longitudinal clinics. Fellows were responsible for the teaching and assessment of the students. We interviewed all stakeholders. Using a grounded theory approach and conversations with a key informant, we illuminated issues and propose best practices.

Results: Students and fellows endorsed success. The students experienced a supportive learning environment with positive role modeling and felt they received good quality feedback. The fellows cited students' contagious enthusiasm and learning different skills as a clinic "junior attending"; many agreed they would continue to teach in clinic. Attending physicians had a mixed view. Those who had not taught students prior felt ill - prepared, while those who regularly teach students welcomed the addition. All agreed that the current model was untenable, due to the lack of organization.

Conclusion: Having GIM fellows as teachers in the internal medicine ambulatory clinic is pedagogically sound, as it offers benefits to both sets of learners. In addition, faculty development is occurring at the level of the fellow, so as to recruit future clinical teachers in the concept of a developing community of practice. However, the operationalization was unsuccessful due to the chaotic nature. We propose a different organization which we hope will be a more sustainable solution. 
DP 25-5 The Impact of Urban-based Family Medicine Postgraduate Rotations on Rural Preceptor/Teachers (IMPORT)

Douglas Myhre University of Calgary, Rebecca Malhi University of Calgary, Jodie Ornstein University of Calgary, Molly Whalen-Browne University of Calgary

Background/Purpose: While the international focus has been the impact of rural rotations on the urbanbased postgraduate learner, there are other stakeholders involved who should be considered. Within the Canadian context, an underserviced rural community may in fact be responsible for training an urban-based postgraduate learner. The impact of these rotations on the supervising preceptors has yet to be examined. This research focuses on seeking to understand the impact of rural rotations for urban post-graduate learners on the rural preceptor. It adds to the literature that seeks to evaluate educational initiatives implemented to respond to the social mandate of serving underserved populations.

Methods: A mixed methods design was used. Data collection started with an electronic survey followed by a voluntary semi-structured interview. The survey was designed based on a literature review and refined by a focus group of experienced rural teaching physicians. The interview tool was refined using principles of grounded theory on emerging themes and continued by an interviewer not affiliated with the program until saturation was achieved. Thematic analysis was completed using a constant comparative approach.

Results: All rural preceptors (75) that have responsibility to supervise urban based residents in the UCalgary Fam Med residency were surveyed. Over $60 \%$ response rate was achieved and from these 15 interviews were completed. There was no difference in preceptor opinion based on demographics. Interview thematic analysis revealed three significant impacts on rural preceptors stratified into personal, professional and financial. Mitigating and coping strategies were identified.

Conclusion: Urban based residents have a significant impact on rural preceptors that has heretofore not been quantified.

\section{DP 25-6 Clinical Learning Experiences and Resident Gender}

Sonya Lee University of Calgary, Sudha Koppula University of Alberta, Stephen Mintsioulis University of Calgary, Maureen Topps University of Calgary, Sarah Jacobs University of Calgary

Background/Purpose: Research suggests that there are differences in clinical learning experiences and that these differences are influenced by resident gender. This study was conducted to understand family medicine residents' experiences and perspectives regarding clinical learning experiences based on resident gender.

Methods: Focus groups with second year family medicine residents from 3 academic communitybased teaching clinics in Calgary were completed between July 2018 and June 2019. Convenience sampling achieved variation in participant gender. Focus groups were audio recorded and transcribed. Transcripts were reviewed by 3 investigators and subjected to thematic analysis with constant comparison.

Results: Focus groups with 23 residents; 11 men and 12 women. Residents who where men described less clinical exposure to patients of other genders (e.g. women's health and obstetrics). The reverse was generally not the same for residents who were women. Other Influencers included a preceptor's patient population, patient preferences, the clinical context/acuity context in which patients were seen, and how the resident was introduced to the patient. Residents responded differently to their experiences and 3 categories were identified: idealistic, pragmatic, and fatalistic. Impact to residents was described as current (loss of confidence, feeling unwelcome) and future (less likely to pursue a particular clinical area).

Conclusion: Education programs and clinical teachers should be aware that resident gender is not the only factor that influences clinical learning experiences. Preceptor and patient factors are important. Residents perceive that how they are introduced to the patient matters. Differences in clinical learning experiences based on resident gender has impact. 
DP 25-7 Addictions 101 for Primary Care: Piloting an Addictions Curriculum with Family Practice Residents

Jennifer Wyman University of Toronto, Ethan Cohen University of Toronto, Anne Wideman University of Toronto, Andrea David University of Toronto, Joyce Nyhof-Young University of Toronto, Mira Shuman University of Toronto

Background/Purpose: Family practice residents typically receive little training in the management of alcohol and opioid use disorders through their residencies. Not all teaching sites have faculty expertise or clinical opportunities for residents to acquire the skills necessary to manage addiction related issues. To address this gap, we piloted an addiction medicine curriculum with residents affiliated with one teaching site of the Department of Family and Community Medicine.

Summary of the Innovation: Our program involved four 2-hour teaching sessions over a two-year period using a "flipped classroom" approach; learners were expected to review specific topic modules online, followed by face to face sessions focusing on casebased discussions, facilitated by a family physician with addictions expertise. Each session focused on a specific substance use issue. Patient management resources were distributed after each session and a repository of resources was maintained online. Participants completed post teaching session surveys. Increases were identified in all measured parameters: confidence in identifying patients with alcohol and opioid use disorders and in offering brief counseling interventions to patients with alcohol and opioid use disorders; likelihood of prescribing anti-craving medications for patients with alcohol use disorder, and likelihood of prescribing buprenorphine for patients with opioid use disorder.

Conclusion: A relatively brief educational intervention can lead to increased confidence in managing alcohol and opioid use disorders amongst family practice residents. The materials used for this pilot could easily be shared with different teaching sites to reduce the workload for facilitators. Followup measures to assess practice patterns would be beneficial
DP 25-8 Reflection and Renewal: A Critical Review of the Teaching Residents to Teach Program for Family Medicine Residents at the University of Toronto

Heather Zimcik University of Toronto, Marcus Law University of Toronto, Jennifer McCabe University of Toronto, Stuart Murdoch University of Toronto, Azadeh Moaveni University of Toronto, Risa Freeman University of Toronto

Background/Purpose: Canadian accreditation standards in both Undergraduate and Postgraduate education now include an expectation of support for residents to develop competency as teachers. Developed in 2001, the Department of Family and Community Medicine at the University of Toronto's Teaching Residents to Teach Program (TRT) is one of the longest running resident-as-teacher training programs in Canada. Given significant changes in national curricular design, and the evolving role of resident teachers in undergraduate medical education, we undertook a critical review of the TRT to help inform future iterations and innovations.

Summary of the Innovation: A three-pronged approach was employed to provide an evidenceinformed process of program evaluation. This included: analysis of 18 years of student evaluations on individual modules; a comprehensive literature review to examine current best practices regarding content and processes; and a survey of recent graduates to better understand the current resident experience with the program.

Conclusion: Evidence of highly positive evaluations of TRT over 18 years confirmed ongoing resident satisfaction with content and format. Recent graduates reported regular engagement and positive experiences in a wide range of teaching settings. Identified barriers to enhancing teaching opportunities included disparity among sites in a distributed curriculum, competing resident priorities, and a need for improved processes to connect residents with available opportunities. The results of this evaluation process will facilitate an evidenceinformed approach to the development and delivery of future TRT modules both locally and nationally. 


\section{DP 25-9 Representation of Women} Presenters at Psychiatry Grand Rounds

Mandy Esliger Dalhousie University, Lara Hazelton Dalhousie University, Heather Milliken Dalhousie University, Kim Good Dalhousie University, Aditya Nidumolu Dalhousie University

Background/Purpose: Despite increasing numbers of women in medicine, the proportion of women compared to men in academic medicine remains low. Buell $D$, et al. identified an under-representation of women presenters in medical grand rounds (BMJ Open, 2018). Psychiatry has traditionally attracted a higher percentage of women than many other specialties. We sought to determine whether there is equal representation of men and women at Dalhousie's Dept. of Psychiatry grand rounds.

Methods: Grand rounds data from 248 presentations between Fall 2012 and Spring 2019 were categorized and analyzed according to gender of presenter. Rounds types included Clinical Academic Rounds (CAR), University Rounds (UR), Child and Adolescent (CA), and the annual R.O. Jones Lecture (ROJL). When not known, gender was inferred from presenter names and additional demographic information available through internet searches. When presentations had multiple presenters, those with at least one female were included in the number of presentations by women.

Results: Overall, women were represented in $52 \%$ of grand rounds presentations. Presentations by women occurred in $64 \%$ of CAR and $56 \%$ of CA. However, women were significantly under-represented in the more prestigious UR, which are offered by invitation to senior academics from other centres $(27 \% p=$ 0.0001). For the Department's major annual lecture (ROJL), there was equal representation of men and women $(43 \%, p=0.27)$.

Conclusion: Women are equally represented in Psychiatry grand rounds but are under-represented as University Rounds speakers. Further analysis would help to clarify reasons for this disparity.

\section{DP 26-1 Mapping Clinical Relevance} Network

Kuo-Hsing Kuo University of British Columbia, Joyce Leo Department of Laboratory Medicine, Royal Jubilee Hospital

Background/Purpose: Clinical relevance is the gold standard to assess whether basic science delivery content is in line with clinical knowledge development. To achieve meaningful learning, delivery content needs to be applicable to build clinical competence. Many attempts have been made to restructure the delivery of basic science in coordination with clinical case-based learning formats. However, the current approach appears to only address re-scheduling of delivery timing, without truly revamping the content to meet the need for clinical knowledge development for the modern healthcare landscape. The challenge lays primarily with navigating through the complexity of clinical knowledge to filter and develop content that is deemed clinically relevant. To achieve meaningful learning, it is essential to generate a comprehensive tool to assist content developers in effectively visualizing levels of clinical relevance.

Methods: In the current pilot, we utilized social network analytical algorithms to identify key influencers to map the connectivity of basic science contents specifically related to histology and pathology, to routine practice, red-flag symptoms and disease prevalence.

Results: We have developed a comprehensive tool to visualize the connective network of clinical knowledge with basic science content.

Conclusion: The development of a clinical relevance network utilizing social network analytical algorithms assisted content developers and learners to navigate and sift through the mountains of clinical knowledge to focus on clinically relevant content. 
DP 26-2 Sipping from the firehose: organizational readiness for big data in learning analytics

David Topps University of Calgary

Background/Purpose: Precision Medicine, Precision Education, Precision, has caught the imagination of many in medical education leadership. Extending the concept of data-driven approaches to personalized learning has its merits but are we ready for this? Despite increasing reliance on technology and painful lessons from malware strikes against our infrastructure, our CRAWWLA project clearly showed the widespread attitude: it's somebody else's problem. Attitudinal ambivalence extends to privacy concerns: learners raise concerns about their schools examining their workflows too closely and yet happily share vicarious moments of their personal lives with known malfeasants such as Facebook.

Summary of the Innovation: We developed DSIP: a framework for assessing and addressing the challenges of organizational readiness, as we move towards the use of big data in learning analytics. Data readiness (micro level) - what data are needed, where to find it, is it valid? System readiness (meso level) where is data stored, is it protected, is it integrated? Institutional readiness (macro level) - regulatory compliance, who pays for it? Personal readiness (mono level) - is it relevant, useful, worth the effort? Is my identity threatened? As we take a few cautious sips from the data firehose that threatens to drown us, we can put the appropriate measures in place to make these transitions palatable.

Conclusion: Built upon the TIRRE model for research readiness, this four level model helps the leadership, and the future participants, at various levels from program to department to faculty, to anticipate the upcoming challenges and explore potential solutions.
DP 26-3 How can the experiences of stakeholders with doctors inform medical selection and education? An interpretive phenomenological study.

Marise Lombard Griffith University, Marise Lombard Griffith University

Background/Purpose: Whilst there is evidence of attempts to address the increasing complexities of selecting and educating tomorrow's doctors, little research has focused on the experiences of those stakeholders most affected by medical practice and malpractice, particularly patients. This study aims to address gaps in the medical student selection and education literature, as well as in the stakeholderbased literature.

Methods: A qualitative approach, utilising phenomenological hermeneutics to explore participants' 'real life' doctor experiences via 39 individual and eight group semi-structured interviews. Data were audio recorded, transcribed verbatim, anonymised and imported into computer assisted qualitative data analysis software. An analytical methodology based on Smith's (1996) Interpretative Phenomenological Analysis (IPA) was applied iteratively to yield two broad phenomenological themes of positively and negatively evaluated doctor experiences.

Results: The most compelling participant experiences were captured verbatim as part of the study's analytical commentary. Positively evaluated experiences focused on doctors 'being patientcentred' and 'good communicators', whilst negatively evaluated experiences focused on doctors 'being unprofessional' and 'compromising their own safety as well as that of others'.

Conclusion: The study impacts ongoing attempts to better inform medical student selection and education. It offers strategies to refine the range and prioritisation of capabilities for enduring and effective medical practice from a more inclusive and unique stakeholder perspective. 
DP 26-4 Elicit 'Rich Data' with 'Rich Pictures': An example exploring palliative care learning in rural Canada

King Keely Northern Ontario School of Medicine, Frances Kilbertus Northern Ontario School of Medicine, Susan Robinson Northern Ontario School of Medicine, Sayra Cristancho Western University, Sarah Burm Dalhousie University

Background/Purpose: Palliative care is complex, challenging and emotional, more-so in rural contexts with limited resources, broad scopes of practice, and relationships entangled across personal-professional barriers. How palliative care learning happens is poorly understood and education efforts must focus on supporting professionals to navigate their unique circumstances. In order to explore how practicing rural physicians and nurses learned to provide palliative care, methods that capture depth and complexity of experience, and how contextual influences are perceived are required.

Methods: Rich picture, a visual research method that uses participant-generated drawings to both evoke and record insights into complex situations, was used in combination with semi-structured interviews to recount narratives of memorable palliative care learning. Participant rural physicians $(n=9)$ and nurses $(n=6)$ were invited to draw their experience followed by an oral exploration of their story.

Results: This combination of methods resulted in vivid, emotional stories. Across all interviews, participants highlighted the role rich pictures played in their ongoing reflections about the particular situations they experienced, as well as the lessons learned. Participants welcomed the opportunity to "put myself in their shoes, in the family's situation" that rich pictures offered and that could not be expressed "just by talking".

Conclusion: Participants described rich picture drawing as enlightening and providing insight into multiple dimensions of their complex experiences that were not evident before the exercise. We suggest this method can be used as a reflection tool to derive deeper understanding of challenging lived experiences.
DP 26-5 There's got to be a better way: Institutional Ethnography of intrapartum practices on a Labour \& Delivery unit

Stella Ng St. Michael's Hospital, Unity Health Toronto, Lori Nemoy St. Michael's Hospital, Unity Health Toronto, Douglas Campbell St. Michael's Hospital, Unity Health Toronto, Filomena Meffe St. Michael's Hospital, Unity Health Toronto, Linda Moscovitch St. Michael's Hospital, Unity Health Toronto, Catherine Bishop St. Michael's Hospital, Unity Health Toronto, Sabina Fella St. Michael's Hospital, Unity Health Toronto, Nirmala Chandrasekaran St. Michael's Hospital, Unity Health Toronto, Ryan Brydges St. Michael's Hospital, Unity Health Toronto

Background/Purpose: Labour and delivery (L\&D) units can be contested workplaces with tensions between obstetrician, nursing, and midwifery practices. These tensions can impede communication and raise patient safety concerns. Remedying such tensions requires study of the driving forces contributing to these recurrent problems in interprofessional practices.

Methods: Our institutional ethnography study initially used critical incident analysis reports to identify recurrent issues related to transfers of care (TOC) and consultation requests between midwives and obstetricians. We then mapped the work of midwives, nurses, family physicians, and obstetricians by observing (75 hours) and interviewing them $(n=15)$. We also traced work processes to local (forms and hospital policies) and external texts (national policies and evidence-based guidelines). Final analysis made visible the otherwise hidden links between everyday work of L\&D practitioners and higher-level coordinators.

Results: We identified three work processes involving midwives consulting obstetricians: induction with TOC back to midwife, consultation without TOC, and TOC. Three points of disjuncture complicated these processes: a local "3 consult rule", linked to higherlevel medical-legal governance and remuneration structures; subjective interpretations of the $4 \mathrm{~cm}$ dilation rule", a policy meant to standardize practice; and regulations that delayed timing of consultations. 
Conclusion: Our study extends existing research demonstrating that medicine-driven governance of midwifery practices can perpetuate interprofessional challenges. For example, the "3 consult rule" was established for patient safety purposes, but in practice can actually function to delay and disrupt work processes, potentially compromising patient safety. While practitioners spoke of the three points of disjuncture as 'laws', most also viewed them as ostensibly modifiable.

\section{DP 26-6 Analyser les interactions} patient/médecin lors de téléconsultations : comprendre la pratique pour mieux former

Sylvie Grosjean University of Ottawa, Luc Bonneville University of Ottawa, Richard Waldolf Institut du Savoir Montfort / Université d'Ottawa, Isaac NahonSerfaty University of Ottawa

Background/Purpose: La télémédecine soulève des enjeux de formation liés à la réorganisation de la communication patient/médecin et au fait que l'information sensorielle est modifiée et redistribuée - voire compromise - par l'utilisation d'un dispositif de télémédecine (Maslen et Lupton, 2018). L'impossibilité de toucher une plaie, de palper un abdomen peut compromettre la capacité du médecin à poser un diagnostic (Miller, 2003). L'objectif de notre étude est d'étudier la réorganisation de l'activité communicationnelle et sensorielle lors de téléconsultations médicales afin de développer un outil de formation.

Methods: En collaboration avec le laboratoire de simulation d'un hôpital ontarien, un scénario de téléconsultation d'un suivi postopératoire en orthopédie a été conçu. Dix médecins ont réalisé ce scénario et ont été filmés afin d'analyser les interactions patient/médecin lors d'un examen clinique à distance. Ensuite des entretiens d'autoconfrontation ont été réalisés produisant un cadre d'interprétation de la pratique clinique par les médecins eux-mêmes.

Results: L'analyse multimodale des téléconsultations et des entretiens nous a permis d'identifier les stratégies mises en oeuvre par les médecins afin de créer une co-présence à distance rendant possible l'évaluation sensorielle du patient, à savoir : (a) procéder à un cadrage de l'interaction établissant une proximité à distance; (b) établir un cadrage clinique via des engagements corporels et (c) réaliser un guidage instructionnel soutenant l'évaluation sensorielle à distance.

Conclusion: Ce travail d'analyse est un préalable nécessaire au développement d'une formation qui permettra aux médecins - à partir de la présentation de situations d'interaction - de se familiariser avec les stratégies identifiées au cours de la recherche.

\section{DP 26-7 Technology and clinician-learner interaction: How is the introduction of a new electronic health record expected to affect educational practice?}

Julianna Caon University of British Columbia, Kevin Eva University of British Columbia

Background/Purpose: The electronic health record (EHR) is an increasingly common platform used in medical settings to capture and store patient information. The implementation of EHRs has been shown to be both positively and negatively impactful in terms of provision of clinical care, depending on the domain analyzed, suggesting that such implementation should not be assumed to be a neutral event. Sociomateriality theory suggests that EHRs can also play a role in workplace-based learning, but very little has been published to date about how EHR implementation affects educational practice from the point of view of clinician-learner interaction. Better understanding such effects will allow better support of learners and educators during clinical transitions to EHRs.

Methods: Using a grounded theory approach to data collection and analysis, semi-structured interviews were conducted with a group of practicing oncologists who work in outpatient clinics while also providing education to medical student and resident trainees. Data regarding perceived impact on the teaching dynamic between clinicians and learners were collected prior to implementation of an EHR to lay the groundwork for more extensive study.

Results: Physician educators perceived that EHR implementation will impact educational practice for both teachers and learners. First, EHR 
implementation is expected to influence teachers' enthusiasm and interest in teaching as well as their ability to teach as much as they want or in a way they feel best supports trainee-learning. Second, EHR implementation is expected to influence learners by changing what is taught, what is modelled, and their role and involvement in both clinical care and the educational dynamic.

Conclusion: Understanding the anticipated impacts of EHR implementation offers potential to allow positive changes to be made that might ease the disruptions caused by future implementation and provides a foundation from which to assess educational impacts during implementation research.

\section{DP 26-8 Use of Simulation in Canadian Undergraduate Medical Schools}

Stephen Miller Dalhousie University, Stuart McAdam Dalhousie University, Tasha Kulai Dalhousie University, Babar Haroon Dalhousie University

Background/Purpose: The study objective was to determine the current usage of simulation based medical education (SBME) in Canadian undergraduate medical schools, as well as to elicit associate deans' (or designate) views on the use of simulation in medical schools. The current state/usage of simulation in Canadian medical schools is not known

Methods: All 17 Undergraduate Medical Education Associate Deans of Canadian medical schools were surveyed on the use of SBME at their respective schools. The survey consisted of a combination of yes/no, multiple choice, and Likert-scale questions. Nine out of the 17 schools completed the survey for a response rate of $53 \%$. Survey results were collected between March 21, 2017 and March 21, 2018

Results: Rich data highlighted all aspects of simulation revealing uubiquitous use in multiple domains. Higher fidelity simulation was commonly associated with team, communication and interprofessional competency-based outcomes. Medium to lower fidelity simulation was more commonly associated with formative learning experiences and procedural skill competency attainment. Schools have begun to use simulation for summative assessment in the new era of CBME. Most agreed that learners should have proficiency via simulation prior to clinical exposure and all agreed of its importance to highlight IPE team work and collaboration.

Conclusion: This study may help guide curriculum choices for Canadian medical schools as they develop experiential learning. Respondents highlighted task trainers/hi-fidelity simulators and simulated patients as future development areas in their curricula.

DP 27-1 Longitudinal Evaluation of Peer Assessment of Professional Competence in Undergraduate Medical Education

Nicholas Fairbridge Memorial University of Newfoundland, Vernon Curran Memorial University of Newfoundland, Diana Deacon Memorial University of Newfoundland

Background/Purpose: Memorial University introduced formative peer assessment into the undergraduate medical education curriculum and adopted Dannefer et al's (2005) professional competence scale. Applying the Norcini et al., (2018) framework for good assessment, we evaluated the use of peer assessment in undergraduate medical education.

Methods: A preliminary survey of medical students' perceptions of peer assessment indicated broad support for anonymous feedback, from peer learning groups, with use restricted to formative and not summative assessment. Students were assessed anonymously by peer learning groups and received peer feedback on four occasions during pre-clerkship phases. Students were surveyed on their satisfaction with the program.

Results: 2756 peer assessments were collected, evaluating 241 students from 3 classes over 4 stages of training. Factor analyses validated Dannefer et al's original professional competence scale and interclass correlations demonstrated strong reliability with 6 or more peer raters. However, assessment items display strong ceiling effects that limit the utility of this scale to chart formative improvement. Students were satisfied with the scale's ease of use, but expressed uncertainty about the educational effects of peer assessment or ability to catalyze professional improvement. 
Conclusion: Peer assessment introduces students to the process of evaluating one's peers and fosters engagement in reflection on professionalism qualities. However, Dannefer et al's professional competence scale may be limited as a tool for longitudinal monitoring of professional competence development and formative improvement. Practical limitations existed in second year where small group size may have impeded peer assessment reliability and anonymity.

\section{DP 27-2 Teaching Professional Practice: "Escape Room" Style}

Karen Sauve University of British Columbia, Alison Greig University of British Columbia, Sue Murphy University of British Columbia, Riley Louie University of British Columbia, Joseph Anthony University of British Columbia

Background/Purpose: Professionalism competence is a requirement in Canadian Physical Therapy entryto-practice standards. The UBC Master of Physical Therapy (MPT) Program professionalism curriculum has evolved; though, student engagement in these courses is limited and professionalism concerns on clinical placements continue to rise. The aim of the "Escape Room" was to provide an engaging 'gamification' of critical curriculum in an innovative learning opportunity.

Summary of the Innovation: A pilot "Escape Room" activity was run with 12 volunteer MPT students in collaboration with UBC's Theatre Department. Two student groups worked to complete a discharge plan while moving through three rooms staged to represent different settings (e.g. hospital room, charting room) with standardized patients. The students worked together to uncover clues during each time-limited interaction. Students targeted issues of professionalism, scope of practice, conflict resolution and ethical decision-making.

Conclusion: Students completed questionnaires immediately after the "Escape Room" activity. Overall, the feedback was positive; students indicated they were engaged through this novel approach to demonstrating their professionalism competence and felt encouraged to be more creative, compared to examination situations. The staging of the rooms was realistic and the actors were well-trained, which further encouraged authentic interactions. Constructive feedback suggested that smaller groups are preferred, a "prize" would increase 'gamification', and clarification of team member roles would improve the experience. This activity provided a successful and engaging approach to practicing and demonstrating professional competencies.

\section{DP 27-3 Tacos with Teachers...Increasing the Connection Between Learners and} Faculty

Ellen M. Friedman Baylor College of Medicine, Toi Harris Baylor College of Medicine, Erik Malmberg Baylor College of Medicine

Background/Purpose: Tacos with Teachers, a collaboration between the Center for Professionalism and the Office of Institutional Diversity, Inclusion and Equity, is an activity where learners and junior faculty meet with respected Baylor faculty members for informal small group conversations that provides learners with insight on how senior faculty find meaning in their careers, what keeps them connected to the joy in their work and strategies behind their disappointments and success. These breakfasts allow learners to hear the stories behind our most successful and senior faculty as they openly share challenges they have faced throughout their careers.

Summary of the Innovation: Faculty members from diverse academic and personal backgrounds were identified as role models and invited to lead a session, which includes breakfast tacos for limited participants to guarantee an intimate setting and accessibility to senior faculty. Response to the sessions has been positive, as found through an anonymous survey. $94 \%$ felt the faculty gave valuable advice on managing adversity based on their experiences. 93\% felt there was appropriate opportunity to ask the faculty teacher questions. $90 \%$ felt the teacher gave useful advice for how to manage one's personal like while still pursuing one's passion. $100 \%$ reported they would recommend this activity to others.

Conclusion: This activity helps build a sense of community among the learners and the Faculty. Attendees appreciate the insights that senior faculty 
offer on resilience and work-life integration, and have found the sessions very valuable in setting realistic expectations for themselves and gaining wisdom from their "elders."

\section{DP 27-4 It's not about being 'nice': A Serious} Illness Communication Curriculum for Cardiology Residents

Katie Marchington University of Toronto, Warren Lewin University of Toronto

Background/Purpose: For patients living with advanced cardiac illness, treatment options are increasingly complex. University of Toronto ( $U$ of $T$ ) cardiology residents have no formal teaching on serious illness communication despite the expectation of competency related to goals of care discussions. Family physician clinician teachers with fellowship training in Palliative Care (PC) are uniquely suited to teach communication as a skill.

Summary of the Innovation: A new serious illness communication skills curriculum, including a 3-hour workshop with standardized patients, was piloted with four first-year $U$ of $T$ cardiology residents and led by two PC clinician teachers. Residents were asked to commit to practicing one skill learned in the workshop in clinical practice and will receive followup coaching by email. Curriculum development was informed by knowledge transfer and deliberate practice theory, and this guided the selection of skills and teaching methods from VitalTalk, an evidencebased communication curriculum.

Conclusion: Residents completed a pre-workshop needs assessment survey indicating they had only 'sometimes' or 'rarely' been observed leading family meetings. In the post-workshop survey, all residents 'strongly agreed' that explicit labeling of communication skills and facilitated reflection during role play helped them learn. All residents agreed they would recommend the workshop to other cardiology residents. Future work will focus on recruiting cardiologists as co-facilitators, which would allow for direct observation of resident communication skills in clinical settings. This project highlights a unique opportunity for family physicians and specialists to collaborate in medical education, and also to reflect on teaching competencies across disciplines.
DP 27-5 Factors influencing credibility of instructors in a faculty development program about leadership

Lara Hazelton Dalhousie University, Susan Love Dalhousie University, Lisa Bonang Dalhousie University

Background/Purpose: Despite increasing interest in leadership education for physicians, there is a lack of information regarding what contributes to the credibility of instructors. Understanding the perceptions of learners would be helpful in selection and training of instructors, and may improve program outcomes.

Methods: A program evaluation survey which included questions about factors affecting instructor credibility was sent to 89 participants from 8 cohorts of an online faculty development course in medical leadership. Descriptive statistics were used to summarize the data, and narrative comments were analyzed thematically.

Results:28 subjects completed the survey. Qualifications for leadership instructors rated most highly (very important or essential) were content expertise related to work experience (84\%) and formal leadership experience (71\%). Less important were medical degrees or degrees in administration (e.g. MBA). Having a PhD in medical education or another field was considered relatively unimportant. Narrative comments supported the findings above, and additionally emphasized that credibility was influenced by instructor behaviors, such as responsiveness, asking probing questions, and making insightful contributions to the discussion boards. The value of including instructors from a variety of backgrounds was also mentioned.

Conclusion: Participants completing an online faculty development course on leadership rate expertise and experience (particularly leadership experience) more highly than academic credentials when assessing instructor credibility. These findings are currently being used to inform instructor recruitment for leadership programs at our school. 
DP 27-6 Exploring Perceptions of Physician Leadership and Character Development Among Doctors, Nurses and Allied Health Members

Jacqueline Torti Western University, Ali Inayat Western University, Hamza Inayat Western University, Wael Haddara Western University, Nabil Sultan Western University

Background/Purpose: Leadership is recognized as an important aspect of a competent physician and has been incorporated into multiple physician competency frameworks. However, the understanding of what physician leadership entails within the medical context remains uncertain and varied. The purpose of this study was to explore health care professionals' perceptions of physician leadership.

Methods: This qualitative descriptive study used semi-structured interviews to explore interdisciplinary health care teams' perceptions of physician leadership with regards to character, competence, and commitment. All interviews were audio-recorded, transcribed and a qualitative latent content analysis of the transcripts was conducted.

Results: A total of 20 interviews were conducted with physicians $(n=5)$, a nurse practitioner $(n=1)$ and allied health members $(n=14)$ within the division of nephrology. Participants identified many traits they deemed to be important to effective physician leadership including a physician's commitment, competence and character, in particular, collaboration, accountability and humanity. A lack of character, in particular, a lack of integrity, humility, humanity, were deemed to contribute to ineffective leadership, along with a lack of commitment and a lack of competence.

Conclusion: This research has demonstrated that health care professionals believe that character, competence and commitment all play a vital role in effective physician leadership. In the era of competency-based design, identifying the perceived value of character and commitment in addition to competency with regards to effective physician leadership provides unique insights with implications for medical schools admission processes, undergraduate and postgraduate medical curricula (both formal and hidden) and ongoing professional development.

\section{DP 27-7 Le profil du professionnel de la} santé socialement responsable: le point de vue des citoyens

Emmanuelle Careau Université Laval, Marie-Claire Bérubé Université Laval, Émélie Provost Université Laval, Julien Poitras Université Laval

Background/Purpose: Selon les principes de responsabilité sociale, les facultés de médecine ont maintenant l'obligation de former les futurs professionnels de la santé à agir de façon adéquate dans la société et à collaborer pour réformer le système de santé afin qu'il soit pertinent, de grande qualité, efficient, équitable et durable au bénéfice des communautés locales, du pays et la communauté internationale. Mais qu'est-il attendu concrètement qu'un étudiant développe comme compétences particulières à cet égard? Actuellement, les profils de compétences plus génériques (ex. CanMEDS) ne sont pas assez détaillés en matière de responsabilité sociale pour permettre aux programmes de bien cibler sur quoi agir pour former des professionnels plus socialement responsables.

Summary of the Innovation: Pour répondre à cette question, la Faculté de médecine de l'Université Laval a organisé trois forums citoyens, dans trois régions différentes du Québec, afin de co-construire avec les citoyens le profil de compétences du professionnel de la santé socialement responsable. À l'aide d'une approche de type world café, les citoyens ont identifié des éléments de compétences qu'ils jugeaient "socialement responsables " à partir de situations qu'ils avaient personnellement vécues. Une analyse qualitative de contenu a ensuite été menée afin de regrouper ces éléments et proposer un profil de compétences spécifique à la responsabilité sociale.

Conclusion: Le profil de compétence du professionnel socialement responsable proposé est composé de sept thèmes : 1 ) intelligence émotionnelle et sociale 2) partenariat avec l'usager 3) collaboration interprofessionnelle 4) pensée systémique de la santé 5) pensée systémique de gestion 6) ancrage dans le milieu 7) éthique et engagement social. 
DP 27-8 An Online Training Program for Clinicians using Telemedicine: From Research to Design

Sylvie Grosjean University of Ottawa, Richard Waldolf Institut du Savoir Montfort, Luc Bonneville University of Ottawa, Judith Boileau Institut du Savoir Montfort, Isaac Nahon-Serfaty University of Ottawa, Maria Cherba Université de Montréal

Background/Purpose: The adoption of telemedicine in healthcare has lead to an increased need for training programs for physicians, medical students, and other clinicians who use telemedicine to provide patient care. Challenges related to affective distance, remote body, and the transformation of sensory perceptions can make a clinical diagnosis more challenging. Most online training programs focus on technological skills rather than communication and relational skills, which are fundamental to putting patients at ease. There is a gap in the development of online training programs focused on these skills.

Summary of the Innovation: In a previous study, we analysed patient/doctor interactions during teleconsultations and reported specific communication strategies and relational skills used by physicians to conduct a remote clinical exam. Based on these results, we are designing an online training program that cover two sets of skills: (a) relational skills to establish a sense of connectedness with the patient and (b) communication skills to compensate for the lack of physical contact. To properly address these skills, we are developing a multimodal learning environment that allows instructional elements presented in more than one sensory mode (visual, auditory, written). The use of multimodal resources is a powerful way to facilitate reflection in and on action for improving relational and communication skills.

Conclusion: The design of an online training program integrating multimodality in a virtual learning environment supports 'reflexivity-in-practice' and provides practical tips for health care providers to communicate effectively with patients during a telemedicine consultation.
DP 27-9 A Workshop to Advance Communication and Primary Palliative Care Skills of 4th Year Medical Students

Tamara Vesel Tufts Medical Center, Grace Wang Tufts medical center, Maria Blanco Tufts University School of Medicine, Emma Ernst Tufts University School of Medicine

Background/Purpose: There is an increasing need for palliative care services and training as the aging population continues to grow along with progress in medical technology. We designed, implemented and evaluated an innovative workshop to advance 4th year medical students' palliative care skills.

Summary of the Innovation: We offered a day-long workshop to fifty 4 th year medical students with the goal of enhancing their communication skills with patients with advanced illness, families and an interdisciplinary team. The curriculum was hands-on, case-based and uniquely structured by immediate, individualized, and real-life feedback from professional actors, interdisciplinary faculty, and a bereaved family member until students demonstrated the core competencies modified from the American Academy for Hospice and Palliative Medicine. Cases included patients of all ages in varying life-threatening situations. We administered pre-, post- and second post-workshop surveys, asked students to report critical learning points, analyzed quantitative survey data, and performed qualitative thematic analysis via inductive open coding. Students reported increased comfort levels with understanding and utilizing palliative care skills and processes and personal qualities for communicating with patients, families and healthcare teams. Students reported having practiced these skills during clinical rotations after the workshop.

Conclusion: Participating students advanced their palliative care skills and gained insights on personal qualities that health care professionals must adopt to communicate appropriately with patients, families and healthcare teams. Since our pilot study yielded promising results, up-scaling and further evaluation of this innovative workshop can potentially lead to a standardized curriculum for medical students nationwide. 
DP 28-1 UBC Indigenous Patient Voice: An Innovative Standardized Patient Session to Develop Indigenous Cultural Safety and to Facilitate Indigenous Instructor Recruitment and Retention

Leah Walker University of British Columbia, Rebecca Howse University of British Columbia, Rebekah Eatmon University of British Columbia

Background/Purpose: The Truth and Reconciliation Commission recommendations on health call for the provision of cultural competency for all health-care providers. The UBC Faculty of Medicine has implemented an Indigenous standardized patient session as part of the Year 4 curriculum in order to ensure that students are able to demonstrate cultural safety in a clinical scenario.

Summary of the Innovation: The standardized patient sessions are lead by health care practitioners with an interest in Indigenous Health. These leaders practice cultural safety themselves, and are able to lead rich discussions on Indigenous health. A critical aspect of the session is that the standardized patients are female community members who authentically represent an Indigenous patient, and are therefore able to elicit holistic and relational health care concerns that are challenging to address in typical medical school curricula. The session also facilitates a process of recruiting instructors interested in helping students practice cultural safety. In order to build a sustainable environment for delivering TRC recommendations, the Faculty of Medicine aims to recruit and retain Instructors and Faculty versed in Indigenous health, who are best positioned to engage students and impart their knowledge to the current faculty.

Conclusion: The 2019 session was highly rated by the medical students in terms of attaining knowledge and skills for practicing cultural safety. The 2020 session will evaluate the student's ability to practice cultural safety by the standardized patients
DP 28-2 Learning by listening to patients: Infusing Patient Voice into Online Education

Yan Chow University of British Columbia, Kate Campbell University of British Columbia, Claudia Hopkins University of British Columbia, Andrea Keesey University of British Columbia, Brenna Lynn University of British Columbia

Background/Purpose: Gender diverse patients need to be heard. Historically treated through a psychiatrist's lens, they are underserved in health care, facing significant disparities like higher rates of poverty, homelessness, unemployment, depression, and substance use. Physicians play an important role. When providers offer non-judgemental and knowledgeable care, gender diverse patients are more likely to continue seeking medical recommendations. A capacity for listening is integral to a gender-affirming, trauma-informed, and patientcentred approach to care.

Summary of the Innovation: UBC CPD partnered with Trans Care BC to develop a Gender-Affirming Primary Care online educational training series. Key areas of the first module were identified as appropriate for patient stories (based on topic, availability or personal accounts, and patient-perceived impact on care). To balance a learner-centred approach with patient authenticity, stories were collected through patient interviews, synthesized, and patientvalidated. Custom patient-centred interview protocols and evaluation templates streamlined medical concepts, effectively capturing patients' input. Finally, patient volunteers lent their voices to the audio stories embedded in the education alongside scripted text. Learners are encouraged to read and listen to the recordings simultaneously creating a more immersive learning experience that caters to different learning preferences.

Conclusion: Patient stories are often considered extraneous in medical education. Even when included, they are developed as an afterthought, which consequently presents the risk of appearing tokenistic. However, when co-developed with patient input, stories can empower patients, enable physicians to practice managing sensitive discussions, 
and provide an avenue for impacting care through real-life examples of what is meaningful to patients.

\section{DP 28-3 Patients impliqués en éducation} des professionnels de santé (PIEPS) : des besoins de formations communes et spécifiques pour chaque groupe.

Isabelle Burnier University of Ottawa, Salomon Fotsing University of Ottawa, Marie Hélène Chomienne University of Ottawa, Diane BouchardLamothe University of Ottawa,

Background/Purpose: Le terme générique « Patients impliqués en éducation des professionnels de la santé » (PIEPS), utilisé ici, regroupe les patients partenaires, les patients simulés et les patients standardisés qui participent aux différents contextes d'apprentissage et d'évaluation des compétences cliniques des futurs professionnels de santé dans le cadre des études médicales. Les PIEPS sont souvent peu informés de ces divers contextes et peu formés pour répondre aux exigences attendues. Ce projet vise à décrire les compétences requises pour être un PIEPS, et à identifier les besoins de formations spécifiques pour chaque groupe de PIEPS.

Methods: Des entrevues téléphoniques semistructurées et des groupes de discussion ont été menés auprès de 25 PIEPS ayant au moins 3 années d'expérience, puis transcrits, analysés et codifiés à l'aide de Nvivo pour extraire les thèmes émergents.

Results: 5 patients partenaires, 10 patients simulés et 10 patients standardisés ont participé. Les compétences de communicateur et de professionnalisme sont essentielles pour les 3 groupes de PIEPS qui expriment le besoin d'une formation de base décrivant leur place en éducation. Les patients simulés et standardisés ont besoin des compétences d'interprétation du scénario clinique et les patients partenaires des compétences relationnelles pour partager leur savoir expérientiel.

Conclusion: Les participants de cette étude ont identifié des compétences essentielles pour accomplir leurs tâches de PIEPS. Ils suggèrent une formation structurée sous forme de mentorat pour maximiser leurs compétences et les orienter au sein du cursus des études médicales.

\section{DP 28-4 How to design courses in} partnership with patients

Mathieu Jackson Université de Montréal, Annie Descoteaux Université de Montréal, Louise Nicaise Université de Montréal, Philippe Karazivan Université de Montréal, Marie-Pierre Codsi Université de Montréal, Clara Dallaire Université de Montréal

Background/Purpose: The Center of Excellence on Partnership with Patients and the Public (CEPPP) with the Collaboration and Patient Partnership Unit (CPPU) of University of Montreal launched in 2019 an online post graduate (masters) course named Fundamentals of Patient Partnership. This course is composed of 12 interrelated themes from various disciplines and was co-developed with over 20 content experts and patients. This complex interdisciplinary structure called for a differing pedagogical approach to each theme.

Summary of the Innovation: The objective of the team was to convey the essence of each disciplinary field. This required the recognition that a given pedagogical approach is never neutral (Bernstein, 2000). Therefore, the development team worked with the premise that the pedagogical framework (ex: structure of objectives, skills, evaluation, etc.) suggested by the content experts from a given field was the most appropriate for teaching that field. The assumption was that content experts know best how to teach their discipline. The pedagogical framework for each theme was determined through discussions between the development team and content experts, during which the pedagogical framework would gradually emerge.

Conclusion: This pedagogical design approach was later formalised during the development of a training program destined for patients accompanying other patients suffering from similar illnesses in healthcare establishments across Quebec. We present the CEPPP and CPPU's methodology for pedagogical design in partnership with patients. 
DP 28-5 Scoping review of Refugee Health Curriculum in Undergraduate Medical Education (UME)

Andrea Davila-Cervantes University of Alberta, Marghalara Rashid University of Alberta, Helly Goez University of Alberta

Background/Purpose: In 2017, the United Nations reported 68.5 million individuals forcibly displaced worldwide as a result of violence or conflict. Increasing refugee population has led to changes in the provision of healthcare. Historically, health care providers have not received targeted training in refugee health $(\mathrm{RH})$. There has been a worldwide interest in developing educational opportunities for medical students in $\mathrm{RH}$. The aim of our work was to identify and analyze currently existing literature on educational content and methods of delivery in UME curricula related to $\mathrm{RH}$.

Methods: We conducted a scoping review. The search strategy was restricted to English language and scholarly articles. Search was conducted in seven electronic bibliographic databases. Three members of the research team tabulated and summarized the extracted data. Descriptive thematic analysis was used to present the findings.

Results: Of the 717 publications found, 26 met inclusion criteria. All were published between 20032019. Fifteen (57.6\%) were descriptive papers, $3(11.5 \%)$ qualitative, $4(15.3 \%)$ quantitative and $1(3.8 \%)$ mixed methods. One commentary, 1 letter to editor and 1 review paper. Four descriptive themes were identified: 1) Content related to refugees' 2) Educational strategies 3) Learning outcomes and 4) Recommendations.

Conclusion: Including content on $\mathrm{RH}$ in the undergraduate medical curriculum, delivered in active learning, small group methodology, and partnering with community resulted in improved students' clinical skills, communication and knowledge. Systematic and longitudinal inclusion of the topic is recommended to provide effective care delivery for this vulnerable population.
DP 28-6 Community Perspectives: Results from community partners' formal review of the Service Learning program for undergraduate medical students

Karen Cook University of Manitoba, Chelsea Jalloh University of Manitoba, Roger Berrington Canu Service Learning Site, Nina Condo Elmwood Community Resource Centre - Service Learning Community Partner, Felicien Rubayita Welcome Place - Service Learning Site, Ian Whetter University of Manitoba

Background/Purpose: In 2016, Service Learning became a curricular requirement for undergraduate medical students at the University of Manitoba. Since that time, significant feedback has been collected from students re: their Service Learning experience. While many community contacts who work with these students regularly provide informal feedback to the university, this survey collected systematic feedback from community partners involved with Service Learning.

Methods: In June 2019, a survey was distributed to 36 organizations to seek feedback about their experiences working with Service Learning students. Participation in the survey was voluntary. In total, 27 organizations participated.

Results: Respondent feedback could be grouped into two main themes: Logistics, and the Service Learning Experience. About half (52\%) indicated it was "easy" to schedule students for Service Learning; however, students' busy schedules and differences between hours of organization programming and students' availability were identified as considerations. Most respondents described students as "engaged" (70\%) or "somewhat engaged" (26\%) in their Service Learning experiences. Sixty-eight percent of respondents indicated Service Learning raised students' understanding of power and privilege, and systemic oppression.

Conclusion: Seeking feedback from community partners involved with Service Learning provided valuable insights to inform and improve the Service Learning program. Results identified specific areas of the program and logistic improvements to be addressed moving forward. Ensuring processes are in 
place to obtain feedback from community organizations is an important step to strengthen reciprocal, working relationships between university and community organizations. Doing so enhances the Service Learning experience for both students and community partners.

\section{DP 28-7 Rural Longitudinal Integrated Clerkship: An answer to increasing numbers of graduates choosing family medicine \& rural practice locations}

Jill Konkin University of Alberta, Daniel Lemoine University of Alberta, Liam Rourke University of Alberta, Darren Nichols University of Alberta

Background/Purpose: Canada's rural populations are dramatically underserved by family physicians. Traditional medical education exasperates this problem by funneling the majority of graduates into urban specialty practice. Longitudinal integrated clerkships may be one way of improving recruitment to rural generalist practice. Our study aimed to determine if students from the rural Integrated Community Clerkship (ICC) at the University of Alberta were more likely to be in family medicine and/or in rural practice locations than those in the rotation-based clerkship (RBC). The rural ICC program is a 40-week 3rd year clerkship with students learning the core disciplines of medicine in an integrated clinic- and hospital-based comprehensive rural family practice.

Methods: Using the $U$ of A graduation lists from 20092016 and public databases, current practice locations and final career choice for all graduates were compiled.

Results: We examined outcomes for 834 RBC graduates and 124 ICC graduates. The RBC graduates were more likely to establish urban practices ( $87 \%$ vs $13 \%$ rural); whereas ICC graduates were equally likely to establish urban and rural practices ( $51 \%$ vs $49 \%$ ). RBC graduates were split between family medicine (48\%) and specialty practice (52\%); whereas ICC graduates were more likely to be in family medicine (78\%) than specialties (22\%). Overall, ICC students were 3.8 times more likely to establish a rural practice compared to $\mathrm{RBC}$ graduates.
Conclusion: Seven years of data suggest that the longitudinal integrated clerkship is effective in increasing the numbers of rural family physicians and contributing to improving health service delivery for rural communities.

\section{DP 28-8 The Senior Citizen Partnership Program (SCPP): a win-win for both medical students and the elderly generation}

Ute Hauck Curtin Medical School

Background/Purpose: Doctors require exemplary communication skills and the right attitude to care for the growing number of elderly patients. Early exposure to the elderly in medical courses has been shown to help change student attitudes and challenge thinking in stereotypes.

Summary of the Innovation: The SCPP runs for the duration of the MBBS course and aims to introduce students to 'healthy ageing'. Two students are partnered with a senior citizen residing in a retirement facility. The students meet their senior partner a prescribed number of times, forming a partnership rather than viewing them as a patient. During these encounters students enquire about social, psychological, environmental and health impacts on the life of the senior, while practicing communication skills taught in clinical skills. Students also attend debriefing sessions and submit reflections about their encounters addressing relevant learning objectives using a reflective framework. Data collected from debriefing sessions, students' assignments and focus groups demonstrate an increased awareness about the elderly generation, acknowledging that previously held stereotypes and reservations were challenged. Students recognise the diversity in this population and the individual needs of seniors, relevant to them as future doctors. The interaction allows students to overcome initial hesitations to approach the elderly, and fosters relevant communications skills by providing authentic experiences. Surveys conducted at the retirement facilities emphasise that the senior partner benefited from the interaction on many levels.

Conclusion: A programme which enables students to foster a trusting partnership with elderly people 
during early exposure in a medical course can help to change the attitudes of medical students.

DP 29-1 Scholarly Activity as a Selection

Criteria in the Canadian Residency

Matching Service (CaRMS): a review of

published criteria by internal medicine, family medicine and pediatrics programs

Amanda Bell McMaster University, Karl Stobbe McMaster University, Larry W. Chambers McMaster University, Vesa Basha McMaster University, Jessie Brazier McMaster University, Delia Dragomir McMaster University, Meghan Glibbery McMaster University, Hannah Kearney McMaster University, Alison Knapp McMaster University, Daniel Levin McMaster University, Dyon Tucker McMaster University, Seddiq Weera McMaster University, Jorin Lukings McMaster University,

Background/Purpose: It is important for residency candidates to have an understanding of the weight that residency programs give to scholarly activities. Residency program descriptions in the Canadian Residency Matching Service (CaRMS) website were reviewed to determine whether scholarly activities were mentioned as a selection criterion.

Methods: 2019 CaRMS published program descriptions were reviewed for internal medicine, family medicine and pediatrics in all 17 Canadian faculties of medicine. Residency programs that mentioned "scholarly activities" or similar descriptors such as "applied health/clinical research", "quality improvement" or "education research" in their descriptions were identified.

Results: Forty-one percent of the family medicine, $65 \%$ of the internal medicine and $71 \%$ of the pediatric residency programs explicitly stated having interest in applicants with scholarly activities. In Western Canada, $80 \%$ of the internal medicine programs and $60 \%$ of the pediatrics programs included scholarly activity in their CaRMS description of criteria considered in ranking applications. Similarly, in Ontario, $66 \%$ of the internal medicine and $83 \%$ of the pediatrics residency programs mentioned scholarly activity as a desirable accomplishment. In Quebec $100 \%$ of the family medicine and $50 \%$ of the pediatrics programs include scholarly activity in their descriptions.

Conclusion: Students can use data from this study to prioritize their activities to include scholarly work during their undergraduate MD training in order to be as competitive as possible for application to specific residency programs in family medicine, internal medicine and pediatrics. The authors recommend CaRMS website descriptions explicitly list selection criteria for residency programs from all of the CanMEDS physician competencies including scholarly activities.

DP 29-2 Histology Milestones and Exit Competencies for Canadian Undergraduate Medical Education

Karen Pinder University of British Columbia, Jason Ford Sidra Medicine

Background/Purpose: There is a developing international transition to competency-based medical education (CBME) in undergraduate and post-graduate medical training. Competencies define measurable skills and behaviours that are expected of medical students and residents and are attained through a series of stages or milestones throughout training. Canadian medical education competencies, particularly foundational medical science competencies, are under-reported. We here report and demonstrate the clinical significance of competencies for a foundational medical science at the medical school at the University of British Columbia. These examples of clinically mapped competencies will be of interest to, and useful for, educators who are teaching in, exploring, or implementing competency-based medical (and allied health fields) curricula.

Summary of the Innovation: Medical schools across Canada have recently developed and embraced CBME curricula at both the undergraduate and postgraduate levels. Many of the Canadian competencies for post-graduate clinical specialties have been described and/or published, but undergraduate foundational medical sciences competencies remain under-reported. It is important to bridge this gap in order to allow integration of competencies across the continuum of undergraduate medical education to 
post-graduate clinical training. We have bridged this gap through the development of an alignment and mapping of undergraduate foundational medical science competencies to post-graduate clinical skills and scenarios.

Conclusion: We here provide a novel description of the generation and use of milestones and competencies in a core undergraduate foundational medical science (histology) at a Canadian medical school. Significantly, by framing our competencies with exemplars, we clearly and uniquely demonstrate how each of our foundational medical science exit competencies can be mapped to clinical scenarios that both medical students and physicians will encounter.

\section{DP 29-3 Distributed Specialty Medical Education in Canada: A Survey}

Charles Penner University of Manitoba

Background/Purpose: Distributed medical education has been increasingly prominent on the Canadian medical education landscape with the development of distributed rotations, longitudinal clerkships, and family medicine programs. A survey of distributed specialty medical education programs in Canada is not yet available. The current research seeks to remedy this deficiency.

Methods: CaRMS advertisements (2018 R1 match) were evaluated for separately advertised distributed streams. Program directors and/or site leads were identified for each stream. A survey inviting comment on the strengths and the challenges of these programs was circulated. In addition, a cohort of residents matched to distributed streams (2009 2012) were identified and practice locations determined using CAPER data for 2018. The odds ratio of graduates of these programs compared to residents from the main programs practicing in settings other than large cities was calculated.

Results: Twenty-two separate streams were identified matching 45 residents $(1.4 \%$ of matched residents). Psychiatry was the most popular program (10/22). Strengths of distributed programs included the wealth of learning opportunities at these sites along with the ability to provide the requisite learning. The challenges identified focused mostly on the difficulty in engaging faculty regarding committee work and formal academic teaching. The odds ratio of graduates of distributed programs practicing in locations other than large cities when compared to residents trained in the main program was 3.77 .

Conclusion: Distributed specialty residency education still accounts for a very small number of residents trained in Canada but is promising as a way of encouraging practice in distributed settings.

\section{DP 29-4 L'évaluation des impacts de l'enseignement médical décentralisé, perception des acteurs.}

Jean Ouellet Université Laval, Elise Martel Université Laval, Sébastien Turgeon Université Laval, Alexandra Dubé-Louber Université Laval, Julie Fortin Université Laval, Raymond Thibodeau Université Laval, Mathieu Pelletier Université Laval, Diane Comeau Université Laval, Claudine Parent Université Laval

Background/Purpose: L'efficacité de la formation décentralisée pour l'installation des finissants en région rurale est bien documentée et son apport économique est aussi mieux connu. II s'avère que l'impact de formation décentralisée sur les différents acteurs en région est moins documenté dans la littérature. Dans le contexte de l'augmentation de la charge clinique et des pressions gouvernementales sur les réseaux de soins, il nous apparaît prioritaire de mieux documenter les impacts dans ce contexte.

Methods: En 2016, à l'aide d'un sondage en ligne auprès de 1200 professionnels, administrateurs et médecins enseignants, nous avons cherché à documenter les perceptions des acteurs locaux sur 4 dimensions: l'équité, les effets non recherchés, la faisabilité et l'acceptabilité de la formation décentralisée. Le cadre d'analyse des politiques publiques de Morestin nous a servi de modèle. Six milieux de formation en région offrant chacun plus de 100 périodes de stages par année de niveau externat et résidences ont participé au sondage.

Results: Nous avons obtenu 301 répondants, dont 76 $\%$ étaient impliqués dans l'enseignement. Les perceptions négatives de l'enseignement sur le milieu étaient faibles, ex: la sécurité des soins (12\%), qualité des soins (10\%). Les perceptions d'effets favorables étaient nombreux et majoritaires pour le 
développement des compétences (78 \%), le recrutement médical (68\%), le développement de l'interdisciplinarité (68\%), la satisfaction au travail (61 $\%)$. Finalement, $81 \%$ des répondants considéraient que globalement l'enseignement médical avait des retombées positives dans leur milieu de travail.

Conclusion: Les perceptions de l'enseignement médical par les acteurs en région qui ont répondu au sondage sont majoritairement positives. Des effets non directement recherchés comme le développement de l'interdisciplinarité et de la compétence des acteurs du milieu sont des avantages indéniables. Les perceptions plus partagées des impacts sur l'acquisition de ressources financières et équipement devraient éveiller notre réflexion. Des sondages plus courts augmenteraient probablement le nombre de répondants. Les impacts sur l'interdisciplinarité, la qualité et la sécurité des soins sont des sujets intéressants à approfondir dans l'avenir.

\section{DP 29-5 Development of an Innovation Procurement Framework for Clinicians}

Angela Coderre-Ball Queen's University, Nancy Dalgarno Queen's University, Jessica Baumhour Queen's University, Iris Ko Georgian College, Vittoria Zubani Queen's University

Background/Purpose: There are increasing pressures on the Canadian healthcare system to provide quality care in the face of an aging population and growing rates of chronic diseases, all while managing escalating costs. To stimulate development of innovative solutions to achieve better health outcomes and optimize the cost of delivering care, healthcare systems in Canada (and in Europe) are beginning to leverage innovation procurement strategies (IPS). Unlike traditional procurement that focuses on providing a solution at the lowest price, in IPS, the procuring organization focuses on understanding the problem, identifying who would be impacted, and defining health outcomes to be achieved. However, the newness of IPS means that few healthcare professionals (HCPs) have the required training to participate in IPS.

Methods: To understand the educational needs of Ontario HCPs, we used a grounded theory approach.
Semi-structured interviews were conducted with 33 HCPs, Ontario health agencies and procurement centres with varying experience in IPS. Transcribed interviews and focus groups were analyzed using thematic design. Open coding was used to allow themes to emerge from the data.

Results: Three overarching themes emerged: IPS processes, building IPS capacity, and supports and resources. From these themes, an IPS clinical framework was developed. The framework includes five components: health innovation culture, IPS process mechanisms, internal supports and resources, and external supports and resources involving vendors/industry and procurement experts.

Conclusion: This IPS clinical framework can be used as a foundation for healthcare agencies wanting to engage in their own IPS, and adds to the dearth of literature in this field.

DP 29-6 Démarche d'intégration des
patients partenaires en évaluation des
étudiants médecine et en sciences de la
santé. L'exemple des étudiants en
médecine en fin d'externat à l'Université de

\section{Montréal.}

Clara Dallaire Université de Montréal, Philippe Karazivan Université de Montréal, Marie-Pierre Codsi Université de Montréal, Annie Descôteaux Université de Montréal, Mathieu Jackson Université de Montréal, Audrey L'Espérance Centre d'excellence sur le partenariat avec les patients et le public

Background/Purpose: Depuis quelques années, I'approche du partenariat de soins s'établit comme étant une référence en pédagogie, en recherche, en gestion et dans les soins. L'évaluation des compétences des étudiants et des soignants par des patients prend elle aussi de plus en plus d'importance en pédagogie et dans les milieux de soins.

Summary of the Innovation: La Direction collaboration et partenariat patient (DCPP) de I'Université de Montréal travaille depuis quelques années à l'intégration des patients partenaires en évaluation des compétences des étudiants en médecine et en sciences de la santé. Ce processus nécessite une démarche rigoureuse débutant par le 
recrutement des patients, leur formation aux fondements du partenariat de soins ainsi que leur formation à l'évaluation des compétences concernant entre autres la posture à prendre en évaluation ainsi que l'utilisation des différents outils d'évaluation. Afin d'illustrer la démarche d'intégration des patients partenaires en évaluation, nous donnons l'exemple de l'examen de reprise ou de remise dispensé aux étudiants de médecine en fin d'externat, depuis les raisons et les circonstances qui ont permis cette innovation pédagogique, le processus de recrutement des patients jusqu'à l'évaluation des étudiants lors de l'examen.

Conclusion: Les différentes étapes du processus d'intégration des patients partenaires en évaluation sont importantes afin d'appuyer la démarche et ainsi permettre une expérience optimale pour tous les acteurs.

\section{DP 29-7 From Learner to Scholar:} Diversifying the Pool of Medical Educators through a PGME Track

Taryn Taylor Emory University School of Medicine, Ulemu Luhanga Emory University School of Medicine

Background/Purpose: Increasingly, postgraduate trainees are in search of additional training to enhance their roles as Scholars. Traditionally medical education tracks have been limited to individual training programs which does not promote collaborative practice across a diverse range of specialties. Emory's Medical Education Residency Track (MERT), is an 18-month program, designed for multidisciplinary trainees to gain competencies essential to becoming medical educators.

Summary of the Innovation: MERT is distributed into three phases: Principles of Scholarly Teaching, Medical Education Scholarship, and Scholarly Project. Sixteen trainees from 11 residency/fellowship programs applied for the 2018-2019 cohort. Eleven were accepted and 10 (91\%) completed Phase 1. Phase 1 involved adopting Kolb's experiential learning model to support trainees' learning through peer teaching. As part of a longitudinal, formative evaluation, we used descriptive statistics and content analyses of survey data to explore the effectiveness of Phase 1. Strengths of Phase 1 included opportunities for group discussions and interactive team work activities. Residents also reported being able to apply skills from sessions about Teaching \& Facilitating Learning (70\%), Learning Needs \& Outcomes (50\%), Bed- \& Bench-side Teaching Strategies (50\%), and Feedback \& Contemporary Feedback Models (50\%). Resident examples of applying skills in their settings included "Using $1 \mathrm{~min}$ preceptor with interns on my service", "utilized Kahoot! during my lectures with Med tech students", and "establishing learning needs and outcomes".

Conclusion: Adopting an innovative peer teaching aspect coupled with using Kolb's 4-stage model not only reinforces learning, but also creates a cadre of future medical educators in a "train-the-trainer" type model. 